Universidade de São Paulo

Instituto de Física

\title{
Simulações de sensores de gás nanoscópicos baseados em nanotubos de carbono: estrutura eletrônica e transporte de elétrons
}

\author{
Amaury de Melo Souza
}

Orientador: Prof. Dr. Antônio José Roque da Silva

\section{Comissão Examinadora:}

Prof. Dr. Antônio José Roque da Silva (IF/USP)

Prof. Dr. Sylvio Roberto Accioly Canuto (IF/USP)

Prof. Dr. Fernando Lázaro Freire Júnior (PUC-RIO)

Dissertação de mestrado
apresentada ao Instituto de
Física para a obtenção do
título de Mestre em Ciências.

titulo de Mestre em Ciências.

São Paulo 


\section{FICHA CATALOGRÁFICA}

\section{Preparada pelo Serviço de Biblioteca e Informação \\ do Instituto de Física da Universidade de São Paulo}

Souza, Amaury de Melo

Simulações de sensores de gás nanoscópicos baseados em nanotubos de carbono: estrutura eletrônica e transporte de elétrons.

São Paulo, 2001.

Dissertação (Mestrado) - Universidade de São Paulo.

Instituto de Física. Depto. Física dos Materiais e Mecânica.

Orientador: Prof. Dr. Antônio José Roque da Silva

Área de Concentração: Física

Unitermos: 1. Estrutura eletrônica; 2. Cálculo ab-initio(DFT);3. Nanotubos de carbono; 4. Propriedades de Transporte eletrônico; 5. Sensores de gás.

USP/IF/SBI-012/2011 
"Grandes coisas fez o Senhor por nós, pelas quais estamos alegres".

Salmos 126:3 



\section{Agradecimentos}

Agradeço de todo meu coração à minha família: à minha mãe Odete, ao meu pai Juanil, aos meus irmãos Aélbany e Daniel e à Fernanda, pelo amor, apoio e confiança que me foram dedicados em todos os momentos. Aos novos amigos da igreja do Sumaré, os quais foram essenciais durante todo o período que estive em São Paulo, que considero como minha segunda família. Aos Professores Antônio José Roque da Silva, Adalberto Fazzio e Alexandre Reily Rocha, pelas orientações e ensinamentos prestados para o desenvolvimento dessa dissertação de mestrado. Aos amigos do grupo SAMPA, pelas discussões, paciência e oportunidades de aprendizado nesse período. Por fim, agradeço ao CNPq pelo apoio financeiro durante o desenvolvimento desse trabalho. 



\section{Resumo}

Desde sua descoberta por S. Iijima em 1991, os nanotubos de carbono têm sido considerados um dos materiais nanoestruturados mais promissores para o desenvolvimento de novos dispositivos eletrônicos em escala nanoscópica. Devido à sua alta razão entre a área superficial e o volume, esse material se destaca para aplicações como sensores de gás. No presente trabalho, estudamos através de simulações computacionais, a possibilidade de nanotubos de carbono com defeitos de nitrogênio (os chamados nanotubos $C N_{x}$ ), poderem ser usados como sensores de moléculas gasosas. Na primeira parte do trabalho foram realizados cálculos de estrutura eletrônica baseados na Teoria do Funcional da Densidade (DFT) para diferentes sistemas formados pelo nanotubo e pela molécula. Através de cálculos de energia de ligação, foi possível identificar quais gases poderiam ou não serem adsorvidos à superfície do nanotubo. Dentre as moléculas investigadas, o monóxido de carbono e a amônia mostraram ser as mais facilmente adsorvidas ao nanotubo. Na segunda parte, foram realizados cálculos das propriedades de transporte utilizando o formalismo das funções de Green fora do equilíbrio (NEGF) recursivo. Foi possível concluir que os nanotubos estudados poderiam ser usados para detectar o monóxido de carbono e a amônia. Todavia, em relação à seletividade, os resultados indicaram que não parece possível distinguir essas duas moléculas, caso o sistema fosse inserido em um ambiente contendo uma mistura desses gases. Ainda, foram feitas simulações de nanotubos contendo defeitos aleatoriamente distribuídos, de forma a levar em conta os fatores de desordem característicos de sistemas mais realistas. 



\section{Abstract}

Since their discovery by S. Iijima in 1991, carbon nanotubes have been considered as one of the most promising nanostructured materials for the development of new nanoscopic electronic devices. Due to its high surface area to volume ratio, this material stands out as a candidate for possible gas sensoring applications. In this thesis, we have studied, by means of computational simulations, the possibility of using carbon nanotubes containing nitrogen defects (the so-called $C N_{x}$ nanotubes) as gas sensors. In the first part, we have performed electronic structure calculations based on Density Functional Theory (DFT) of several systems to address the possible binding of different molecules to the nanotube surface. Our results indicate that, among the molecules which were investigated, carbon monoxide and ammonia adsorb more easily to the nanotube surface. In the second part of this thesis, we have performed calculations of the transport properties by means of non-equilibrium Green's function formalism (NEGF). The results have shown that the nitrogen-defect carbon nanotubes could be used to detect, mainly carbon monoxide and ammonia molecules. On the other hand, when dealing with the selectivity of this system, it seems to be not possible to distinguish these gases, in the case of inserting the system in a environment containing a mixture of these molecules. Finally, we have simulated carbon nanotubes with defects randomly distributed along its length, in order to take into account disordering factors usually found in more realistic nanosensors. 



\section{Índice}

1 Introdução: Sensores de Gás 1

1.1 Aplicações . . . . . . . . . . . . . . . . . . . . . 1

1.2 Sensores de gás baseados em nanotubos de carbono . . . . . . . . . . . 3

1.2.1 Motivação e proposta deste trabalho . . . . . . . . . . . . . . . 6

1.3 Estrutura da dissertação . . . . . . . . . . . . . . . . . . . . . . . . 11

2 Nanoestruturas de Carbono $\quad 12$

2.1 Grafeno . . . . . . . . . . . . . . . . . . . . . 12

2.2 Nanotubos . . . . . . . . . . . . . . . . . . . 15

2.3 Propriedades eletrônicas dos nanotubos de carbono . . . . . . . . . . . 18

2.4 Técnicas de crescimento de nanotubos de carbono $(C N T s) \ldots \ldots$. . . . 20

3 Metodologia 24

3.1 Teoria do Funcional da Densidade - DFT . . . . . . . . . . . . . . 24

3.2 Teoria quântica de Transporte eletrônico . . . . . . . . . . . . . . 25

3.2.1 Quantização da Condutância . . . . . . . . . . . 26

3.2.2 Formalismo da Função de Green Fora do Equilíbrio (NEGF) . . . 31

3.2.3 Transporte eletrônico em sistemas desordenados . . . . . . . . . . . 36

4 Propriedades Estruturais e Energéticas $\quad 45$

4.1 Estudo do processo de dissociação de moléculas na região do defeito $N R \quad$. 45

4.1.1 Primeiro grupo de moléculas: Principais constituintes do ar atmosférico . . . . . . . . . . . . . . . . 47

4.1.2 Segundo grupo de moléculas: Alguns gases poluentes . . . . . . . . 53

4.1.3 Resumo dos resultados do estudo do processo de dissociação . . . . 57 
5 Propriedades de transporte eletrônico $\quad 60$

5.1 Resultados individuais para os sistemas nanotubo + molécula . . . . . . . 63

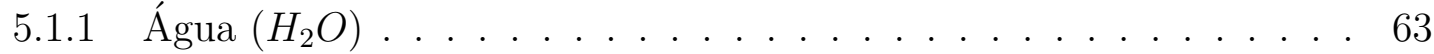

5.1 .2 Ácido Sulfídrico $\left(H_{2} S\right) \ldots \ldots \ldots \ldots$

5.1 .3 Oxigênio molecular $\left(\mathrm{O}_{2}\right) \ldots \ldots \ldots \ldots$. . . . . . . . 67

5.1.4 Hidrogênio $\left(H_{2}\right) \ldots \ldots \ldots \ldots$. . . . . . . . . . . . . 68

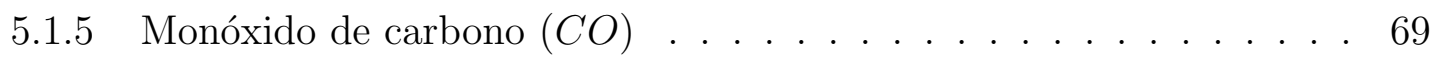

5.1 .6 Amônia $\left(\mathrm{NH}_{3}\right) \ldots \ldots \ldots \ldots$. . . . . . . . . . . 70

5.2 Como tratar a seletividade? . . . . . . . . . . . . . . . . 71

5.3 Propriedades de transporte de sistemas desordenados . . . . . . . . . . . . 74

5.4 Resumo dos resultados das propriedades de transporte . . . . . . . . . . . 78

6 Conclusões e Perspectivas $\quad 80$

A Teoria do funcional da densidade - DFT 85

A.1 Teoremas de Hohenberg-Kohn . . . . . . . . . . . . . . . . . . 86

A.2 Aproximação de Thomas-Fermi . . . . . . . . . . . . . . . . . . . . . . 89

A.3 Equações de Kohn-Sham . . . . . . . . . . . . . . . . . . . . . . . 90

A.4 Aproximações $L D A$ e $G G A \ldots \ldots \ldots$

B Otimização de parâmetros do SIESTA para o nanotubo $(5,5) \quad 94$

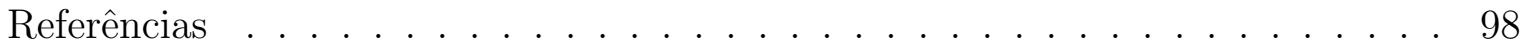




\section{Capítulo 1}

\section{Introdução: Sensores de Gás}

A possibilidade de detectar baixas concentrações de uma espécie química específica como moléculas gasosas, é fundamental para uma variedade de processos que vai desde a indústria, aplicações médicas até monitoramento e controle do meio ambiente [1]. Em particular, sensores de gás podem ser utilizados na prevenção e/ou indicação de possíveis incêndios, vazamentos de produtos químicos, sistemas de monitoramento e detecção da concentração de uma espécie química que apresente alta toxicidade.

\subsection{Aplicações}

$\mathrm{Na}$ indústria automobilística, sensores de gás têm sido empregados em sistemas de ventilação dos carros, os chamados auto-damper ventilation system (ADS) [2], que servem para controlar a qualidade do ar no interior do veículo dependendo se o ar externo está limpo ou poluído. Em geral, os sensores utilizados nos $A D S$ são construídos para detectar alguns tipos de hidrocarbonetos e $N O_{x}$.

Uma outra importante área de aplicação de sensores de gás é a medicina. Existe um consenso na comunidade médica da importância de se desenvolver métodos de diagnósticos e tratamentos não invasivos e uma metodologia que se destaca são as análises respiratórias [3]. Esta técnica se baseia no fato de que existem aproximadamente 200 compostos voláteis no ar exalado durante a nossa respiração, além dos principais constituites $\mathrm{H}_{2} \mathrm{O}, \mathrm{N}_{2}$ e $\mathrm{O}_{2}$. Pesquisas na área indicam que o aumento da concentração de determinadas espécies gasosas no ar exalado pelo paciente está correlacionado com doenças. Como exemplo, podemos citar a relação entre a acetona e diabetes $[4,5]$, alcanos e aromáticos que podem 
estar ligados ao cancêr de pulmão [6] dentre outras [7, 8]. Outras vantagens das análises respiratórias são a rapidez destas quando comparadas com coletas ambulatoriais, fácil intervenção e controle por parte do médico e, principalmente o ganho no tratamento pelo diagnóstico precoce. Desta forma, diversos tipos de sensores com diferentes mecanismos de funcionamento e condições de operação têm sido investigados e produzidos para esse fim.

A poluição de solos, fontes de água e ar devido ao aumento das atividades industriais, exploração de recursos naturais e crescimento da população, traz a necessidade de se fazer o controle e monitoramento do meio ambiente para preservação e desenvolvimento sustentável [9]. Em particular, as poluições atmosférica e da água podem causar desastres ambientais em um curto período de tempo devido à rápida difusão dos contaminantes para grandes áreas. Em relação à qualidade do ar, além do controle ambiental externo, observa-se também uma preocupação maior em se ter um ambiente saudável para viver e trabalhar, o que abre uma série de aplicações para monitoramento e controle do ar de interiores [10]. Por exemplo, detectores de umidade, de gases provenientes de cigarros e de gases produtos de combustão como $\mathrm{CO}_{2}$ e $\mathrm{CO}$ são usados em sistemas de purificação e/ou condicionamento de ar, exaustores e outros equipamentos para casas e escritórios $[11]$.

Na parte de segurança, os sensores conhecidos como nariz eletrônico são utilizados na detecção de compostos orgânicos voláteis ou odores gerados por alimentos e outros produtos utilizados em casas. Um resumo da vasta aplicação para os sensores de gás está mostrado na Tab.1.1.

Os tipos de sensores de gás mais empregados atualmente são os baseados em semicondutores devido a fatores como sensibilidade ${ }^{1}$, estabilidade e custo [12]. Entretanto, existem algumas limitações importantes, como por exemplo:

(i) a faixa de temperatura de operação dos sensores de semicondutores é de 200 a $500^{\circ} \mathrm{C}$, o que é muito acima da temperatura ambiente desejável [13].

(ii) a sensibilidade desses dispositivos é em geral limitada a concentrações de parte por milhão (ppm). Entretanto, alguns gases tóxicos podem causar danos à saúde em concentrações de partes por bilhão (ppb).

\footnotetext{
${ }^{1}$ A sensibilidade é definida como a razão entre a resistência do dispositivo antes da exposição ao gás e a resistência depois da exposição.
} 
Tabela 1.1: Resumo de algumas aplicações para sensores de gás em diversas áreas.

\begin{tabular}{ll}
\hline \hline \multirow{2}{*}{ Automotiva } & Detecção de gás combustível \\
& Testes de teor alcoólico no organismo \\
& Controle de consumo e rendimento do combustível \\
& Sistema de ventilação de carros \\
\hline Segurança & Alarmes de incêndio \\
& Vazamento de produtos químicos \\
& Detecção de gases inflamáveis/tóxicos \\
\hline Qualidade do ar de interiores & Controle de ventilação para exaustores \\
& Controle de cheiro de alimentos (narizes eletrônicos) \\
\hline Controle ambiental & Controle de poluição e monitoramento da qualidade do ar \\
& Monitoramento da umidade do ar \\
& Controle e tratamento de efluentes industriais \\
\hline Medicina & Diagnósticos e análises ambulatoriais \\
& Análises farmacológicas \\
\hline \hline
\end{tabular}

(iii) um outro aspecto é que, em geral, os sensores baseados em semicondutores, mesmo considerando os menores fabricados, estão na escala de micrometros. A fabricação de nano-sensores poderia, por exemplo, desempenhar um papel importante na detecção de moléculas in vivo em aplicações na medicina.

\subsection{Sensores de gás baseados em nanotubos de car- bono}

A nanotecnologia têm desempenhado um papel fundamental no desenvolvimento de novas tecnologias em diversas áreas do conhecimento. Em física de materiais e eletrônica, por exemplo, novos materiais mais resistentes e com propriedades intrigantes têm sido usados na fabricação de dispositivos cada vez menores e mais eficientes. Os sensores de gás fazem parte deste contexto e, portanto, têm-se investido em novas possibilidades de sensores cada vez mais sensíveis, de baixo custo e menores. 
Materiais nanoestruturados como nanotubos de carbono [14], nanofios semicondutores $[15,16]$ e nanofitas de carbono [17] têm sido estudados para possíveis aplicações como sensores de gás. Devido à grande razão entre a área superficial e volume desses materiais, podem ocorrer grandes mudanças em suas propriedades eletrônicas e de transporte eletrônico devido a pequenas quantidades de moléculas externas ao dispositivo. Em aplicações biológicas, esses materiais se mostram como excelentes candidatos a serem usados como sensores e que poderiam operar, inclusive, em temperatura ambiente.

Em relação à possibilidade de aplicação dos nanotubos de carbono como sensores de gás, o primeiro trabalho publicado foi por Kong et al. em 2000 [18]. Os autores demonstraram que nanotubos de carbono semicondutores de parede única ( $S W C N T s$ ) tinham sua condutividade modificada quando expostos aos gases $\mathrm{NO}_{2}$ e $\mathrm{NH}_{3}$ e apresentavam sensibilidade da ordem de $10^{3}$. Também observaram a seletividade do dispositivo; em uma atmosfera contendo $\mathrm{NO}_{2}$ a resistência diminuía, enquanto que na presença de $\mathrm{NH}_{3}$ a resistência do dispositivo aumentava. Ainda em 2000, Collins et al. [19], através de medidas de resistência elétrica e da sensibilidade de nanotubos semicondutores acoplados a eletrodos metálicos, eles observaram que essas propriedades sofriam modificações significativas quando o sistema era exposto a uma atmosfera rica em $\mathrm{O}_{2}$. Em outras palavras, o sistema mudava seu comportamento de semicondutor para metálico quando na presença do gás.

Outros trabalhos experimentais por Katayama et al. [20] e Myung et al. [21] mostraram que filmes de $S W C N T$ s poderiam detectar os gases $\mathrm{NO}_{2}$ e $\mathrm{NH}_{3}$, respectivamente, elevando ainda mais a sensibilidade dos dispositivos. Ainda para o $\mathrm{NH}_{3}$, os estudos também foram estendidos para os nanotubos de paredes múltiplas ( $M W C N T s$ ), e os resultados mostraram novamente que a resistência do sistema decai quando exposto a uma atmosfera contendo esse gás [22].

Estudos teóricos através de simulações computacionais têm sido de grande importância para o desenvolvimento de novos materiais e da nanoeletrônica. A física computacional ajuda a entender os fenômenos observados experimentalmente, a prever novas propriedades e a desenhar novos dispositivos para diversas aplicações. Dentro do contexto de nanosensores de gás, alguns trabalhos teóricos através de cálculos de primeiros princípios baseados na Teoria do Funcional da Densidade $(D F T)$ foram feitos para investigar a adsorção de moléculas nas superfícies de nanotubos de carbono. Por exemplo, Zhao et al. [23] es- 
tudaram a adsorção de várias moléculas $\left(\mathrm{NO}_{2}, \mathrm{O}_{2}, \mathrm{NH}_{3}, \mathrm{~N}_{2}, \mathrm{CO}_{2}, \mathrm{CH}_{4}, \mathrm{H}_{2} \mathrm{O}\right.$ e $\left.\mathrm{H}_{2}\right)$ na superfície de $S W C N T s$ e também em feixes. Os resultados obtidos dos cálculos de energia de adsorção e transferências de carga mostraram que apenas as moléculas $\mathrm{NO}_{2}$ e $\mathrm{O}_{2}$ poderiam ser detectadas pelos $S W C N T s$. Eles também concluíram que não há uma dependência clara entre a energia de adsorção das moléculas com o diâmetro e a quiralidade dos nanotubos.

Motivados pelos resultados experimentais [18] obtidos para o $\mathrm{NO}_{2}$, Dai et al. [24] investigaram teoricamente através de cálculos de DFT a adsorção desta molécula e seus derivados $\mathrm{NO}$ e $\mathrm{NO}_{3}$ na superfície de vários tipos de nanotubos semicondutores. Os resultados mostraram através dos cálculos das energias de adsorção do $\mathrm{NO}$ e do $\mathrm{NO}_{2}$ que estas moléculas não se ligariam e, portanto, não poderiam ser detectadas. O que parece acontecer é que, devido à pequena barreira de difusão destas na superfície do nanotubo, a reação para formação do $\mathrm{NO}_{3}$ é favorável e é este gás que possui energia de adsorção suficiente para ser detectado.

Em relação ao trabalho de Collins et al. [19], Tersoff et al. [25], através de simulações de um FET (Field Effect Transistor) utilizando um nanotubo semicondutor como canal de condução ligado a eletrodos metálicos, observaram que a resposta do dispositivo à variação da voltagem de "gate" para diferentes valores de função trabalho dos eletrodos é idêntica à observada quando o sistema é exposto ou não à atmosfera rica em $\mathrm{O}_{2}$. Ou seja, para certos valores de função trabalho dos eletrodos, o sistema se comportava como semicondutor ou metal. Isso sugere que o gás se ligaria aos eletrodos, uma vez que eles também são expostos ao gás durante o experimento. Isso causaria a modificação da função trabalho e, consequentemente, modificaria a resposta do sistema à voltagem de "gate" aplicada.

Em geral, as propriedades eletrônicas dos nanotubos de carbono puros são sensíveis à exposição a certos tipos de gases. Entretanto, as moléculas que esses sistemas poderiam detectar são aquelas que apresentam energias de adsorção altas suficientes para que possa ocorrer transferências de carga entre a molécula e o nanotubo. Desta maneira, moléculas tais como $\mathrm{CO}$ e $\mathrm{H}_{2} \mathrm{O}$ que possuem energias de adsorção da ordem da energia térmica, não poderiam ser detectadas pelos nanotubos puros. Um ponto importante é que, durante os processos de síntese desses nanotubos, defeitos estruturais podem ocorrer e acredita-se que esses defeitos na superfície dos nanotubos é que funcionariam como sítios para esses 
gases se ligarem [26].

Para tentar contornar essa limitação causada pela baixa afinidade entre as moléculas e os nanotubos puros, Peng et al. [27] propuseram teoricamente nanotubos contendo heteroátomos tais como boro e nitrogênio, o que já estava sendo realizado experimentalmente [28]. Os resultados mostraram que, enquanto os nanotubos puros não detectam as moléculas $\mathrm{CO}$ e $\mathrm{H}_{2} \mathrm{O}$, os nanotubos com os heteroátomos apresentaram energias de adsorção relativamente altas $\sim 0.4 \mathrm{eV}$. A combinação desses fatores poderia resultar em uma engenharia de nanotubos para detectar uma grande variedade de moléculas.

Além de heteroátomos, defeitos de vacâncias de átomos de carbono contendo metais de transição nesses defeitos foram estudados teoricamente por Jacobsen et al. [29]. Os resultados indicaram que a presença de um átomo de níquel, substituindo um átomo de carbono em um nanotubo $(6,6)$, poderia servir como sensor para detectar $C O$. Outros trabalhos teóricos também baseados na DFT estudaram feixes de nanotubos para detectar a presença de $\mathrm{O}_{2}$ e $\mathrm{N}_{2}$ [30] e nanotubos com defeitos de oxigênio [31] para detectar vários gases.

\subsubsection{Motivação e proposta deste trabalho}

A principal motivação para este trabalho vem de um artigo publicado por Terrones e colaboradores em 2002 [32], no qual apresentaram a síntese de nanotubos de carbono contendo defeitos de nitrogênio $\left(C N_{x}\right)$, Fig. 1.1(a). Nesse mesmo artigo, os autores propuseram uma possível configuração dos defeitos que os nanotubos poderiam apresentar, constituída de uma vacância e três nitrogênios substitucionais, conforme mostrado na Fig. 1.1(b). Em 2004 [33], o mesmo grupo fez experimentos em que os nanotubos dopados com nitrogênio foram inseridos em diferentes atmosferas contendo moléculas orgânicas tais como acetona, clorofórmio, gasolina e também amônia. Os resultados mostraram que o sistema nanotubo enriquecido com nitrogênio sofre variações significativas em sua condutividade, causadas pelas moléculas que se ligam nas regiões de defeito, indicando que tais sistemas poderiam ser usados como sensores de gás. Por exemplo, a Fig. 1.2 mostra os resultados experimentais para o sistema exposto a uma atmosfera rica em amônia. A resistência do sistema aumenta devido à presença do gás e volta a diminuir quando o gás é expelido, indicando um comportamente de sensoriamento. Também foram apresentados cálculos teóricos de primeiros princípios para estudar como a molécula de amônia poderia 


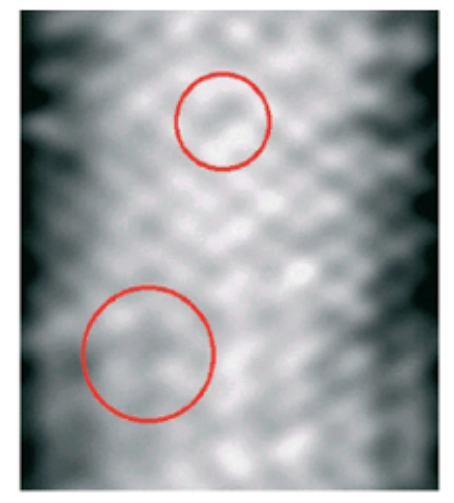

(a)

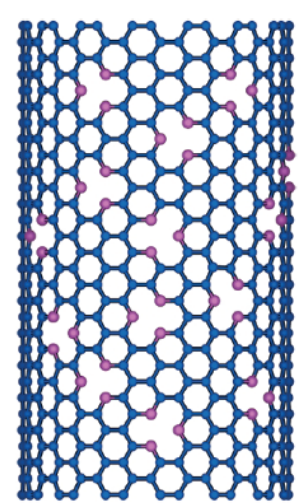

(b)

Figura 1.1: (a) Imagem de STM da superfície de um nanotubo de 20nm de diâmetro dopado com átomos de nitrogênio. Os círculos em vermelho destacam vacâncias. (b) Estrutura proposta para o defeito contendo 3 átomos de nitrogênio substitucionais e uma vacância de carbono. Figuras retiradas da referência [32].

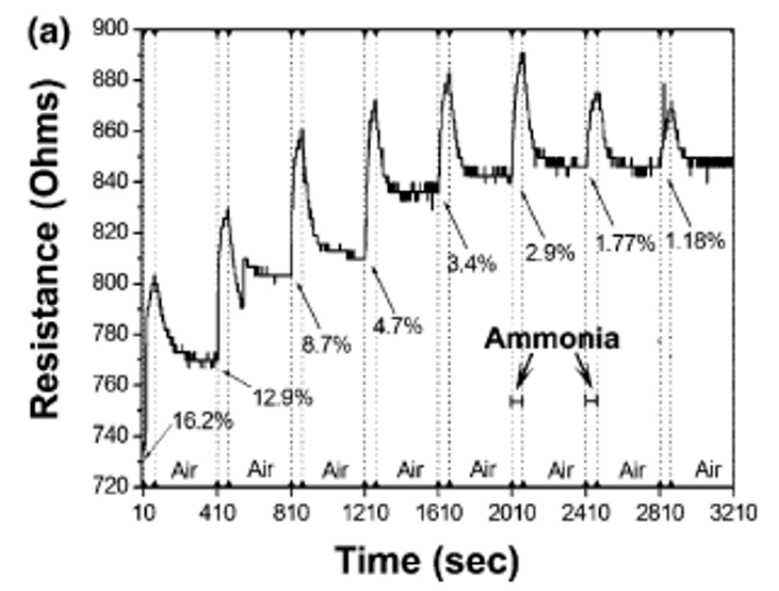

Figura 1.2: Resistência dos $C N_{x}$ em função do tempo de exposição à amônia para diferentes concentrações do gás. Figura retirada da referência [33].

se ligar na região do defeito de nitrogênios em um nanotubo $(5,5)$.

Todos esses resultados apresentados pelo trabalho publicado por Terrones et al. motivaram o estudo detalhado de qual tipo de defeito seria o mais provável no nanotubo $(5,5)$ e como seriam as propriedades de transporte do sistema devido à presença de uma molécula de $\mathrm{NH}_{3}$. Esse trabalho foi realizado no nosso grupo [34]. Nesse trabalho, foram investigados vários tipos de defeitos contendo átomos de nitrogênio, conforme Fig. 1.3. Através de cálculos da energia de formação (Fig. 1.4) em função do potencial químico 


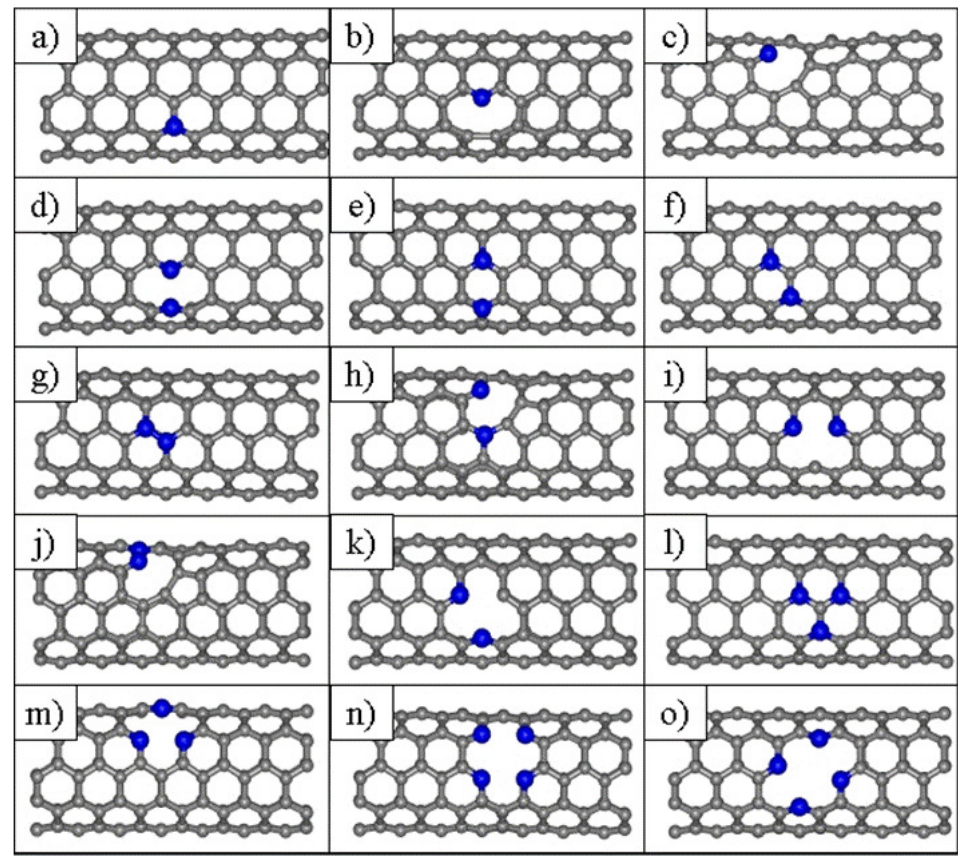

Figura 1.3: Diferentes tipos de defeito de nitrogênio em um nanotubo (5,5). (a) um átomo de nitrogênio substitucional $(1 \mathrm{~N})$; (b) e (c) uma vacância de carbono e um átomo de nitrogênio substitucional (1Nvac); (d) a (g) dois átomos de nitrogênio substitucionais $(2 \mathrm{~N})$; (h) a $(\mathrm{k})$ dois átomos de nitrogênios substitucionais e uma vacância de carbono (2Nvac); (l) três átomos de nitrogênio substitucionais $(3 \mathrm{~N}) ;(\mathrm{m})$ três átomos de nitrogênios substitucionais em torno de um vacância de carbono (3Nvac); (n) quatro átomos de nitrogênio substitucionais em torno de uma vacância de carbono e (o) quatro átomos de nitrogênio substitucionais em torno de uma vacância dupla de carbono (4Ndivac). Figura retirada da referência [35].

para os diferentes defeitos, verificou-se que o tipo de defeito mais provável é o mostrado na Fig. 1.5 (que corresponde à estrutura "o" da Fig. 1.3), já que para a maioria dos potenciais químicos testados, este foi o que apresentou energia de formação mais baixa.

Os resultados de cálculos das propriedades de transporte apresentados por Reily et al. [36] mostraram que o sistema é sensível na detecção da amônia, uma vez que a transmitância do sistema com a presença da molécula sofre variações expressivas. No entanto, os autores apresentaram cálculos das propriedades de transporte para os $C N_{x}$ com a molécula de amônia para dois tipos de defeitos: o primeiro para o tipo de defeito proposto por Terrones et al. [32] mostrado na Fig. 1.1(b) (que corresponde à estrutura "m" da Fig. 1.3) e o segundo para o mais estável, Fig. 1.5. 


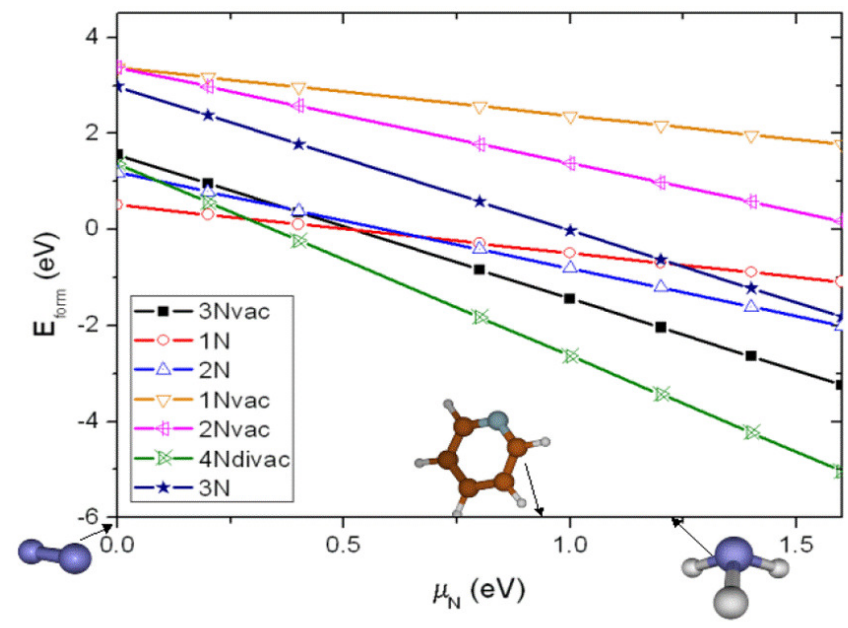

Figura 1.4: Energia de formação em função do potencial químico para diferentes tipos de defeitos mostrados na Fig. 1.3. Os potenciais químicos para diferentes fontes de nitrogênio é destacado. Figura retirada da referência [34].

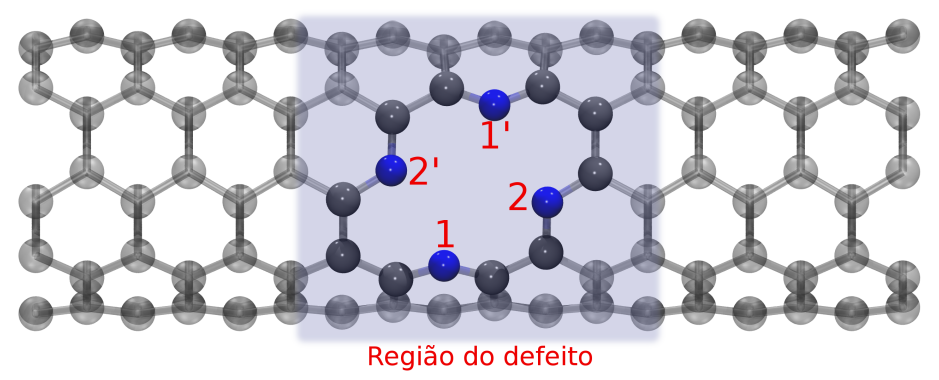

Figura 1.5: Sistema NR para o nanotubo $(5,5)$ contendo 178 átomos. O defeito é formado pela divacância de carbono e 4 átomos de nitrogênio substitucionais. Os rótulos nos átomos de nitrogênio indicam os sítios equivalentes, $1 \equiv 1^{\prime}$ e $2 \equiv 2^{\prime}$.

Através de cálculos de sistemas contendo várias unidades de defeito aleatoreamente distribuídas ${ }^{2}$, foram feitos vários cálculos das propriedades de transporte desses sistemas para diferentes concentrações de moléculas de amônia. Os resultados teóricos corroboraram os resultados experimentais [33] quando o tipo de defeito considerado é o que corresponde à Fig. 1.5.

Diante deste cenário, o presente trabalho tem como objetivo estudar as propriedades

\footnotetext{
${ }^{2}$ Discutiremos em detalhes no capítulo de metodologia como são feitos os cálculos quando consideramos vários defeitos distribuídos aleatoriamente.
} 
eletrônicas e de transporte para o sistema mostrado na Fig. 1.5 (o qual denominaremos $N R$ (nitrogen-rich) nessa dissertação) na presença de cada uma das moléculas mostradas na Fig. 1.6. Desejamos abordar as seguintes questões:

- Através do estudo do processo de dissociação, quais moléculas se ligam na região de defeito $N R$ do nanotubo e como se dá esse processo?

- Através do estudo das propriedades de transporte, é possível saber se uma molécula se dissociou/ligou na região de defeito do nanotubo de maneira que pudesse ser detectada?

- O sistema poderia ser utilizado como sensor de gás? Quais gases poderiam ser detectados se o sistema fosse inserido em uma atmosfera contendo uma mistura de gases?

- Qual a importância de se considerar os fatores de desordem para simulações do sistema $N R$ como sensor de gás?

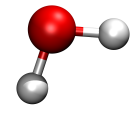

(a) $\mathrm{H}_{2} \mathrm{O}$

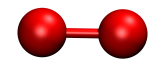

(b) $\mathrm{O}_{2}$

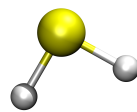

(f) $\mathrm{H}_{2} \mathrm{~S}$ (e) $\mathrm{CO}_{2}$

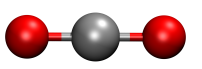

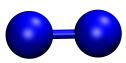

(c) $\mathrm{N}_{2}$

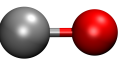

(g) $\mathrm{CO}$

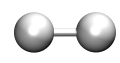

(d) $H_{2}$

Figura 1.6: Moléculas estudadas nesse trabalho: (a) água; (b) oxigênio; (c) nitrogênio; (d) hidrogênio; (e) dióxido de carbono; (f) ácido sulf́́drico; (g) monóxido de carbono e (h) amônia.

A motivação para a escolha das moléculas $\mathrm{H}_{2} \mathrm{O}, \mathrm{O}_{2}$ e $\mathrm{N}_{2}$ baseia-se no fato de que para uma possível aplicação do sistema $N R$ como sensor de gás seria importante que os principais constituintes do ar atmosférico não interferissem nas medidas, ou seja, que estas moléculas não fossem detectadas. Por outro lado, as moléculas $\mathrm{CO}, \mathrm{CO}_{2}, \mathrm{H}_{2} \mathrm{~S}, \mathrm{H}_{2}$ e $\mathrm{NH}_{3}$ são desejáveis de se detectar por várias razões, conforme exposto na seção 1.1. 
Através de cálculos de estrutura eletrônica (DFT implementada no código SIESTA [37]) dos sistemas propostos $N R+$ molécula, deseja-se obter as estruturas de mais baixas energias através da relaxação total do sistema. Uma vez feito este estudo, as estruturas relaxadas serão utilizadas para os cálculos das propriedades de transporte eletrônico para verificar como a dissociação das moléculas na região do defeito modificam a condutividade dos nanotubos.

\subsection{Estrutura da dissertação}

No capítulo 2, faremos uma introdução sobre as nanoestruturas de carbono, discutindo as propriedades eletrônicas do grafeno e como elas podem ser usadas para entendermos as propriedades eletrônicas dos nanotubos de carbono. Será apresentado um pequeno resumo de técnicas de crescimento de nanotubos para contextualizar os nanotubos enriquecidos com nitrogênio, já que estes são os objetos de estudo nesse trabalho.

Para a parte de metodologia, dedicaremos o capítulo 3 para a metodologia de transporte eletrônico através de nanoestruturas que será dividida em duas partes. Na primeira, trataremos das idéias básicas de transporte eletrônico em sistemas nanoscópicos e na segunda abordaremos o formalismo das funções de Green fora do equilíbrio (NEGF) e sistemas desordenados. A metodologia para tratar a estrutura eletrônica $(D F T)$ foi colocada no apêndice A.

Em relação aos resultados, dividiremos em dois capítulos: no capítulo 4 apresentaremos os resultados do estudo do processo de dissociação das moléculas na região do defeito do nanotubo; no capítulo 5, os resultados das propriedades de transporte eletrônico para sistemas com um único defeito e também levando em conta os fatores de desordem.

Finalmente, no capítulo 6, apresentaremos as conclusões do trabalho com os principais resultados retomando as questões que o motivaram. Terminaremos com algumas sugestões de estudos que poderiam ser feitos para corroborarem os resultados obtidos e aprofundar ainda mais no entendimento de mecanismos de detecção de gases pelos nanotubos de carbono. 


\section{Capítulo 2}

\section{Nanoestruturas de Carbono}

O carbono é um dos elementos mais abundantes na natureza e se apresenta em diversas formas alotrópicas. Dentro da área de física dos sólidos podemos citar o grafite e o diamante. Com 4 elétrons de valência, os orbitais $s$ e $p$ do carbono podem se hibridizar de diferentes maneiras resultando em estruturas distintas. A hibridização $s p^{3}$ é a encontrada na estrutura do diamante, enquanto que a $s p^{2}$ é a encontrada no grafite e grafeno. Nos fulerenos e nanotubos, devido à curvatura imposta pela geometria desses materiais, os átomos de carbono apresentam hibridização com carácter $s p^{2}$ e $s p^{3}$. Os fulerenos, nanotubos e grafeno são formas alotrópicas descobertas em 1985 [38], 1991 [14] e 2004 [39], respectivamente.

Existe um consenso na comunidade científica de que a microeletrônica baseada na tecnologia do silício está chegando ao seu limite de miniaturização. Desta maneira, têmse buscado novos materiais que possam ser empregados na fabricação de dispositivos eletrônicos em escala nanométrica. Nesse cenário, as nanoestruturas de carbono têm apresentado grande potencial. Nesse trabalho trataremos dos nanotubos de carbono e de sua possível aplicação como sensores de gás. Entretanto, é útil discutirmos sobre o grafeno para facilitar o entendimento da estrutura dos nanotubos.

\subsection{Grafeno}

O grafite (3D) é a forma alotrópica do carbono mais abundante na natureza e pode ser visto como o empilhamento de folhas de grafeno (2D). Na folha de grafeno, cada átomo de carbono está igualmente ligado a 3 carbonos vizinhos, formando assim, uma 
rede hexagonal bidimensional. Já os nanotubos de carbono, que são estruturas (1D), podem ser entendidos como folhas de grafeno enroladas. Finalmente os fulerenos (0D), através do corte específico de uma parte da folha de grafeno. Ver Fig. 2.1.

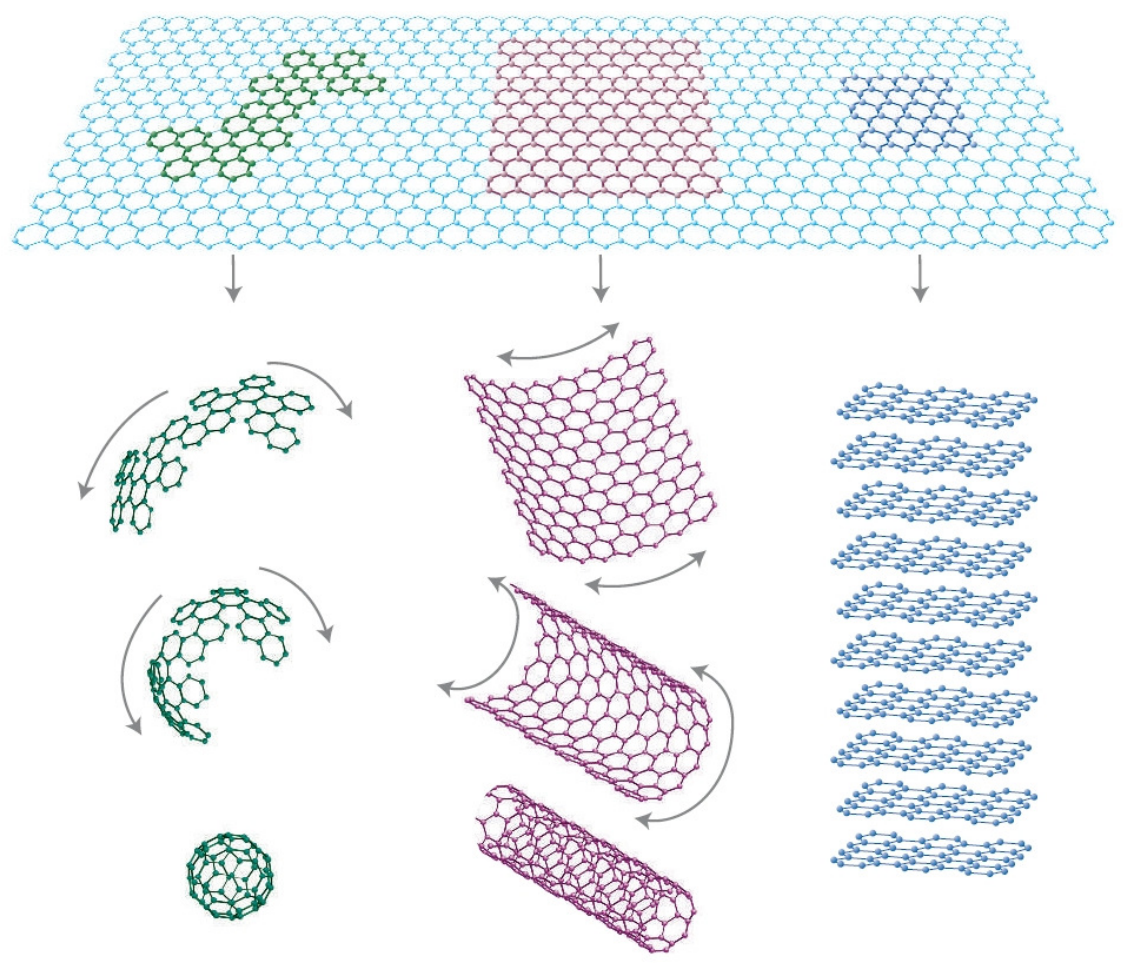

Figura 2.1: Grafeno [40]- constituinte fundamental do fulereno (0D), nanotubo (1D) e grafite (3D).

As primeiras tentativas de isolar o grafeno foram por meio de esfoliação química. Para isso, átomos ou moléculas são inseridos entre as camadas de grafeno de um cristal de grafite de tal forma que sejam obtidas camadas separadas de grafeno.

Também ocorreram algumas tentativas de fazer crescer grafeno, de maneira semelhante à que geralmente se faz para nanotubos de carbono. Porém, obtiveram-se somente filmes finos de grafite com $\sim 100$ camadas de grafeno. Por outro lado, já se obteve o crescimento de algumas folhas de grafeno epitaxialmente, por meio da deposição química de vapor de hidrocarbonetos em substratos metálicos e, também, através da decomposição térmica do SiC. Acredita-se que o processo de crescimento epitaxial de grafeno seja a forma mais promissora de utilização em aplicações eletrônicas [40]. No grafeno, os átomos de carbono apresentam hibridização $s p^{2}$ para os orbitais $2 s, 2 p_{x}, 2 p_{y}$ e $2 p_{z}$ em que o último fica perpendicular ao plano da folha de grafeno. Os orbitais $s, p_{x}$ e $p_{y}$ se combinam para 


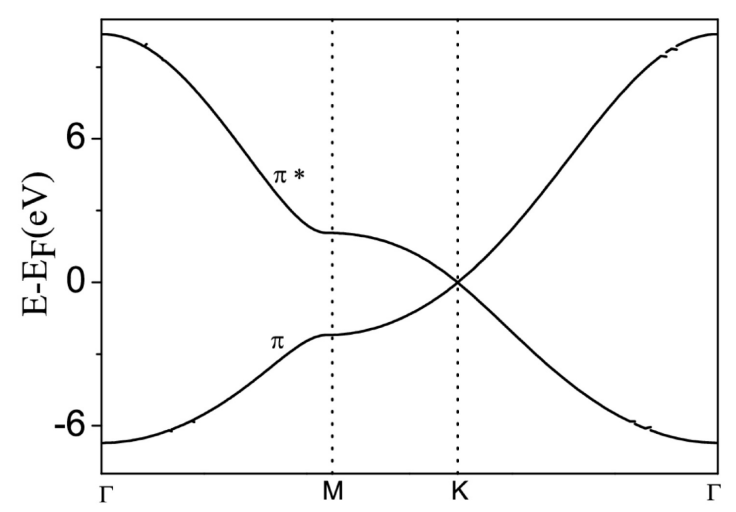

(a)

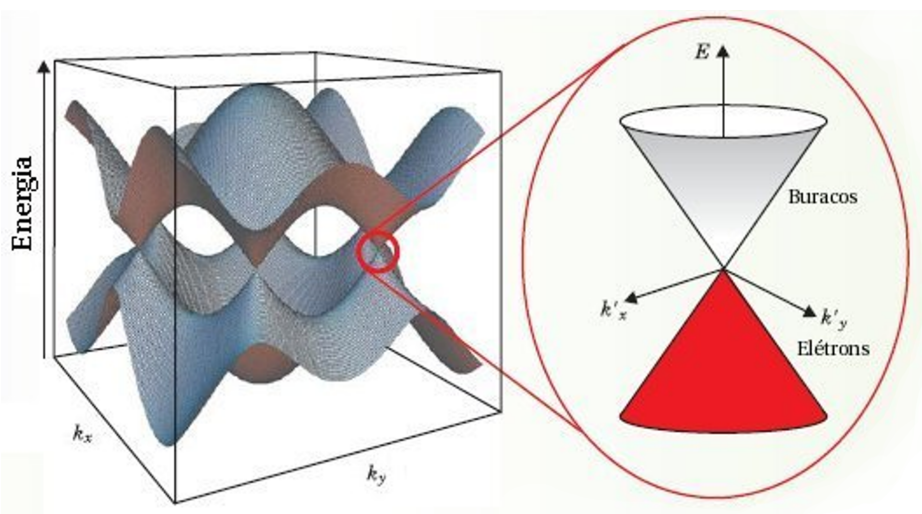

(b)

Figura 2.2: (a) Estrutura de bandas do grafeno calculada com método Tight-biding. (b) Bandas de valência e de condução do grafeno; a dispersão linear em torno do ponto de alta-simetria K no nível de Fermi é conhecida como cone de Dirac.

formarem os orbitais $\sigma$ ligante (ocupado) e $\sigma^{*}$ anti-ligante (desocupado) no plano da folha de grafeno. Esses orbitais são "simétricos" com respeito à simetria de reflexão no plano. As ligações $\sigma$ são ligações covalentes fortes responsáveis pela maior parte da energia de ligação e das propriedades elásticas da folha de grafeno. Já os orbitais $p_{z}$ perpendiculares ao plano da folha são "anti-simétricos " com respeito à simetria de reflexão no plano e não podem se acoplar com os estados $\sigma$. A interação lateral com os orbitais $p_{z}$ dos átomos vizinhos criam estados deslocalizados do tipo $\pi$ ligantes (ocupados) e $\pi^{*}$ antiligantes (desocupados). Quanto à energia desses orbitais, os do tipo $\sigma$ possuem valores característicos de energias muito abaixo do nível de Fermi e, portanto, não desempenham papel fundamental na estrutura eletrônica do material. Entretanto, os orbitais do tipo $\pi$ apresentam energias em torno do nível de Fermi. No ponto $K$ de alta-simetria da zona de Brillouin do grafeno, a banda de energia (orbital $\pi$ ) cruza o nível de Fermi com 
dispersão linear em torno deste ponto (Fig. 2.2). Portanto, dizemos que o grafeno é um semicondutor de gap nulo. O entendimento da estrutura eletrônica do grafeno nos dá o ponto de partida para entendermos a estrutura eletrônica de nanotubos de carbono e para mais detalhes sugerimos o artigo de revisão da referência [41].

\subsection{Nanotubos}

A síntese dos nanotubos de carbono foi anunciada pela primeira vez em 1991 por S. Iijima [14]. Naquele experimento, foram produzidas estruturas com 2 a 50 nanotubos coaxiais (MWNTs), que variavam de 2 a $30 \mathrm{~nm}$ de diâmetro e chegavam a $1 \mu \mathrm{m}$ de comprimento.

Imagens obtidas por microscopia eletrônica de transmissão, Fig. 2.3(a), mostravam a mesma quantidade de linhas em torno de um espaçamento central, o que sugeriu trataremse de tubos coaxiais. Verificou-se que a distância entre duas camadas era $\sim 0,35 \mathrm{~nm}$, semelhante à separação das folhas de grafeno no grafite, e, por meio de uma análise de padrões de difração de elétrons, observou-se que os hexágonos de carbono se arranjavam de forma helicoidal em torno do eixo do tubo.

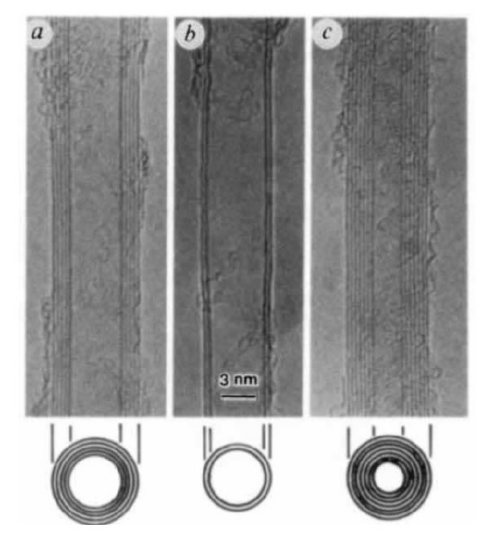

(a)

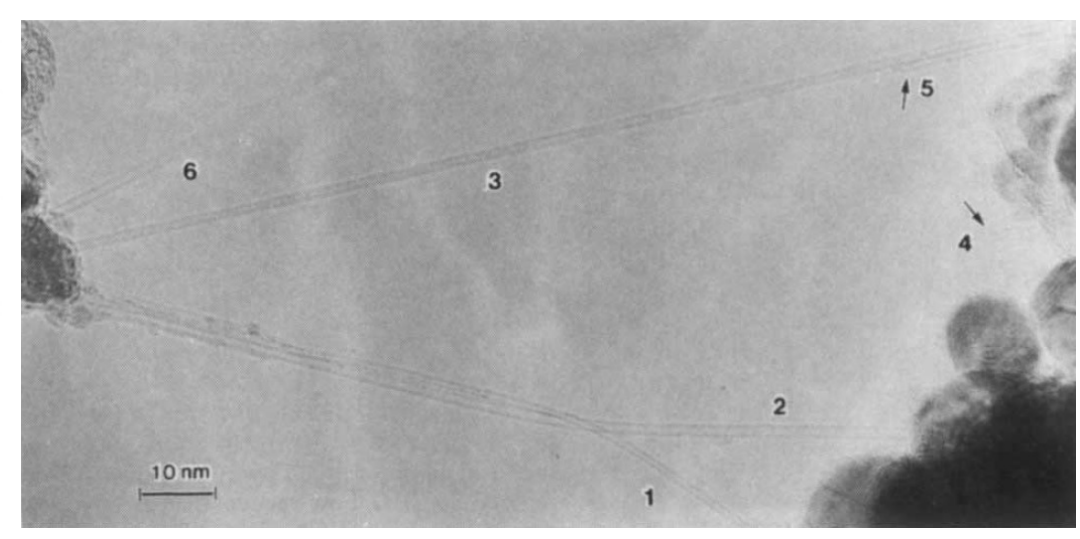

(b)

Figura 2.3: Imagens obtidas por microscopia eletrônica de transmissão: (a) MWNTs [14]; (b) SWNTs com diâmetros de $0.075 \mathrm{~nm}$ (nanotubo 1) e $1.37 \mathrm{~nm}$ (nanotubo 2) [42].

Em um outro artigo publicado em 1993, Iijima anunciou a obtenção de nanotubos de parede única (SWNTs) [42]. O aparato experimental era semelhante ao experimento anterior, porém com a utilização de metais para servirem como catalizadores. Verificouse que a maior parte dos nanotubos de parede única se juntavam formando feixes, mas 
alguns deles foram observados isoladamente, Fig. 2.3(b).

Devido ao fato de os nanotubos poderem ser entendidos como sendo folhas de grafeno enroladas, suas estruturas estão intimamente relacionadas com a estrutura do grafeno e em geral são descritas em termos dos vetores de rede do grafeno. A estrutura do nanotubo é descrita seguindo uma notação introduzida por Hamada [43], em que os nanotubos são representados por dois índices $(n, m)$.

Para representar a rede hexagonal do grafeno é necessária uma célula unitária com dois átomos, A e $\mathbf{B}$ na Fig. $2.4^{1}$, de modo que, ao repetí-la em todos os pontos dados pela combinação linear de múltiplos inteiros dos vetores unitários $\mathbf{a}_{1}$ e $\mathbf{a}_{2}$, recupera-se a rede original.

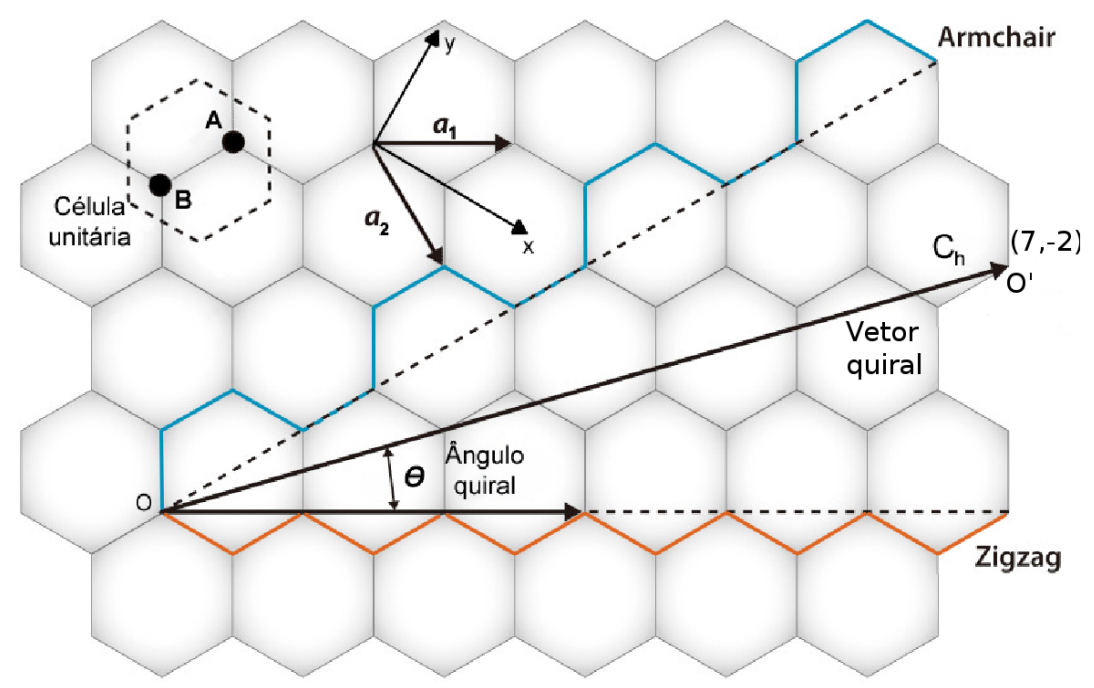

Figura 2.4: Rede hexagonal do grafeno com os vetores unitários $\left(\mathbf{a}_{1}\right.$ e $\left.\mathbf{a}_{2}\right)$ e a célula unitária formada por dois átomos A e B . O vetor quiral $\left(\mathbf{C}_{h}\right)$ mostra o exemplo de como se enrola o grafeno para formar o nanotubo $(7,-2)$. As direções armchair e zigzag estão destacadas em azul e vermelho, respectivamente.

Se a constante de rede do grafeno é $a=\sqrt{3} a_{c c}$ em que $a_{c c}$ é o comprimento de ligação entre átomos de carbono, os vetores unitários podem ser representados no plano $x y$ por $\mathbf{a}_{1}=a\left(\frac{\sqrt{3}}{2}, \frac{1}{2}\right)$ e $\mathbf{a}_{2}=a\left(\frac{\sqrt{3}}{2},-\frac{1}{2}\right)$. Os vetores de base no espaço recíproco $\left(\mathbf{b}_{1}, \mathbf{b}_{2}\right)$ se relacionam com os vetores de base do espaço real por $\mathbf{a}_{i} \cdot \mathbf{b}_{j}=2 \pi \delta_{i j}$; portanto, para a rede recíproca $\mathbf{b}_{1}=b\left(\frac{1}{2}, \frac{\sqrt{3}}{2}\right)$ e $\mathbf{b}_{2}=b\left(\frac{1}{2},-\frac{\sqrt{3}}{2}\right)$ em que $b=\frac{4 \pi}{a \sqrt{3}}$. O vetor quiral dado por $\mathbf{C}_{h}=n \mathbf{a}_{1}+m \mathbf{a}_{2}$, é definido de forma que ele conecte dois pontos equivalentes da

\footnotetext{
${ }^{1}$ Esta figura foi extraída de T. Ando, NPG Asia Materials, 1, 17-21 (2009), e adaptada a este trabalho.
} 
rede cristalina, por exemplo, os pontos $O$ e $O^{\prime}$. Na Fig. 2.4, o vetor quiral é dado por $\mathbf{C}_{h}=7 \mathbf{a}_{1}-2 \mathbf{a}_{2}$. Isto significa que o nanotubo será formado de modo que o átomo de carbono localizado no ponto $O$ seja o mesmo átomo do ponto $O^{\prime}$ após o enrolamento da folha de grafeno. O comprimento do vetor quiral define a circunferência do nanotubo e consequentemente seu diâmetro, que é dado por:

$$
d_{t}=\left|\mathbf{C}_{h}\right| / \pi=\frac{a}{\pi} \sqrt{n^{2}+n m+m^{2}} .
$$

Já a inclinação $\theta$ do vetor quiral define como será o enrolamento helicoidal do nanotubo, ou, equivalentemente, os coeficientes lineares $(n, m)$ tais que $\mathbf{C}_{h}=n \mathbf{a}_{1}+m \mathbf{a}_{2} \operatorname{com} n, m$ inteiros. O ângulo $\theta$ pode ser calculado por:

$$
\cos \theta=\frac{\mathbf{C}_{h} \cdot \mathbf{a}_{1}}{\left|\mathbf{C}_{h}\right|\left|\mathbf{a}_{1}\right|}=\frac{2 n+m}{2 \sqrt{n^{2}+n m+m^{2}}},
$$

em que este ângulo está restrito ao intervalo $0 \leq|\theta| \leq 30$, devido à simetria hexagonal da rede do grafeno. Ainda podemos definir o vetor de translação da rede do nanotubo ao longo do eixo de crescimento do mesmo. Esse vetor $\mathbf{T}$ é o menor vetor da rede do grafeno que é perpendicular ao vetor quiral $\mathbf{C}_{h}$. Em termos dos vetores da base temos que $\mathbf{T}=t_{1} \mathbf{a}_{1}+t_{2} \mathbf{a}_{2}$. Com a condição de perpendicularidade $\left(\mathbf{C}_{h} \cdot \mathbf{T}=0\right)$ temos que as componentes do vetor translação são dadas por:

$$
t_{1}=\frac{2 m+n}{N_{R}}, \quad t_{2}=-\frac{2 n+m}{N_{R}}
$$

em que $N_{R}$ é o máximo divisor comum dos números $(2 m+n)$ e $(2 n+m)$. Desta forma, o comprimento do vetor translação é dado por:

$$
|\mathbf{T}|=\sqrt{3} a \sqrt{n^{2}+n m+m^{2}} / N_{R}
$$

Os nanotubos são classificados em três classes quanto à quiralidade, definida pelos índices $n$ e $m$ : os nanotubos são denominados zigzag se $m=0$; armchair quando $|n|=|m|$ e quirais quando $|n| \neq|m|$. Além de serem extremamente resistentes e essencialmente materiais undimensionais, a estrutura eletrônica dos nanotubos está estreitamente relacionada com a geometria dos mesmos. Por exemplo, os nanotubos armchair são metálicos e uma parte dos zigzag são semicondutores, como discutiremos na seção 2.3. Na Fig. 2.5 estão mostrados exemplos das três classes de nanotubos citados e na Tab. 2.1 está o resumo dos principais parâmetros estruturais dos nanotubos. 
Tabela 2.1: Propriedades estruturais dos nanotubos de carbono.

\begin{tabular}{llll}
\hline \hline Simbolo & Nome & Fórmula & Valor \\
\hline$a$ & constante de rede & $a=\sqrt{3} a_{c c} \simeq 2.46 \AA$ & $a_{c c} \simeq 1.42 \AA$ \\
$\mathbf{a}_{1}, \mathbf{a}_{2}$ & vetores da base & $\left(\frac{\sqrt{3}}{2} ; \frac{1}{2}\right) a,\left(\frac{\sqrt{3}}{2} ;-\frac{1}{2}\right) a$ & \\
$\mathbf{b}_{1}, \mathbf{b}_{2}$ & vetores da rede recíproca & $\mathbf{b}_{1}=b\left(\frac{1}{2} ; \frac{\sqrt{3}}{2}\right), \mathbf{b}_{2}=b\left(\frac{1}{2} ;-\frac{\sqrt{3}}{2}\right)$ & $b=\frac{4 \pi}{a \sqrt{3}}$ \\
$\mathbf{C}_{h}$ & vetor quiral & $\mathbf{C}_{h}=n \mathbf{a}_{1}+m \mathbf{a}_{2} \equiv(n, m)$ & $(0 \leq|m| \leq n)$ \\
$d_{t}$ & diametro do tubo & $d_{t}=\left|\mathbf{C}_{h}\right| / \pi=\frac{a}{\pi} \sqrt{n^{2}+n m+m^{2}}$ & \\
$\theta$ & ângulo quiral & $\cos \theta=\frac{\mathbf{C}_{h} \cdot \mathbf{a}_{1}}{\left|\mathbf{C}_{h}\right|\left|\mathbf{a}_{1}\right|}=\frac{2 n+m}{2 \sqrt{n^{2}+n m+m^{2}}}$ & $0 \leq|\theta| \leq 30$ \\
$\mathbf{T}$ & vetor translação & $\mathbf{T}=t_{1} \mathbf{a}_{1}+t_{2} \mathbf{a}_{2} \equiv\left(t_{1}, t_{2}\right)$ & $t_{1}=\frac{2 m+n}{N_{R}}, t_{2}=-\frac{2 n+m}{N_{R}}$ \\
\hline
\end{tabular}

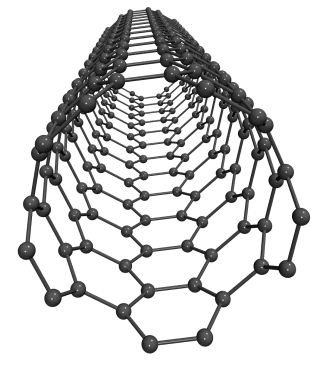

(a)

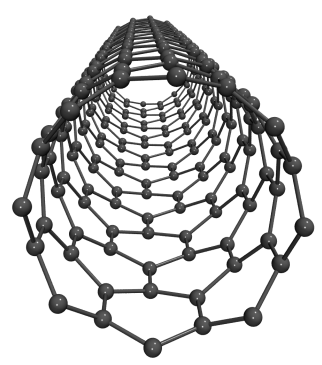

(b)

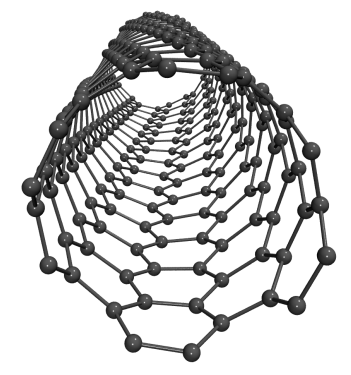

(c)

Figura 2.5: Estruturas geométricas de exemplos das três classes de nanotubos: (a) armchair $(5,5)$; (b) zigzag $(8,0)$ e (c) quiral $(6,4)$.

\subsection{Propriedades eletrônicas dos nanotubos de car- bono}

Devido às condições de contorno periódicas ao longo da circunferência do nanotubo impostas quando o grafeno é enrolado, os vetores de onda ficam quantizados nessa direção. Já os vetores de onda ao longo do eixo de crescimento do nanotubo permanecem contínuos se o nanotubo for infinito. Partindo da Zona de Brillouin do grafeno, os vetores de onda permitidos após quantização devido ao enrolamento da folha são aqueles sobre uma série de retas, conforme discutiremos abaixo.

A estrutura específica de um nanotubo de carbono é determinada pelo vetor quiral 
$\mathbf{C}_{h}=(n, m)$ expresso em termos dos vetores da base $\left(\mathbf{a}_{1}, \mathbf{a}_{2}\right)$ e cujo módulo é $\left|\mathbf{C}_{h}\right|=$ $a \sqrt{n^{2}+n m+m^{2}}$. Ele determina a simetria do nanotubo e o diâmetro. A quantização ao longo da circunferência gera restrições à fase da função de onda, de acordo com:

$$
\Psi_{\mathbf{k}}\left(\mathbf{r}+\mathbf{C}_{h}\right)=e^{i \mathbf{k} \cdot \mathbf{C}_{h}} \Psi_{\mathbf{k}}(\mathbf{r})=\Psi_{\mathbf{k}}(\mathbf{r})
$$

em que o vetor $\mathbf{r}$ é tomado na superfície do nanotubo e o vetor de onda $\mathbf{k}$ na direção perpendicular à direção de crescimento do nanotubo. Podemos perceber que a quantização dos vetores de onda dependem da simetria do nanotubo, que está relacionada com o vetor quiral.

Vamos iniciar a análise das propriedades eletrônicas dos nanotubos olhando as vizinhanças da superficie de Fermi. Façamos uma perturbação no vetor de onda $\mathbf{k}=\mathbf{K}+\delta \mathbf{k}$

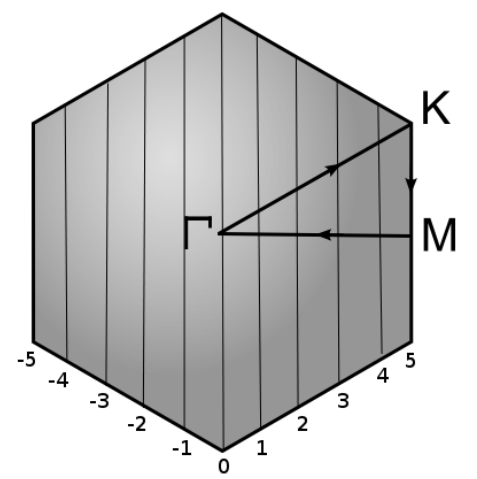

Figura 2.6: $1^{\underline{0} a}$ Zona de Brillouin do grafeno mostrando os pontos de alta-simetria $\Gamma$, $K$ e $M$. As famílias de retas e os números quânticos $q$ associados a essas retas para o nanotubo $(5,5)$.

com $\delta \mathbf{k}$ pequeno, em que $\mathbf{K}=\left(\mathbf{b}_{1}-\mathbf{b}_{2}\right) / 3=(0,4 \pi / 3 a)$. Essa é a condição para que os valores de $\mathbf{K}$ estejam sobre pontos equivalentes ao ponto $K$ de alta-simetria da zona de Brillouin do grafeno. Com a condição de que $e^{i \mathbf{K} \cdot \mathbf{C}_{h}}=1$, devemos ter:

$$
\begin{aligned}
\mathbf{K} \cdot \mathbf{C}_{h} & =2 \pi l \\
\frac{\left(\mathbf{b}_{1}-\mathbf{b}_{2}\right)}{3} \cdot\left(n \mathbf{a}_{1}+m \mathbf{a}_{2}\right) & =2 \pi l \\
(0,4 \pi / 3 a)\left[\frac{a \sqrt{3}}{2}(n+m), \frac{a}{2}(n-m)\right] & =2 \pi l \\
(n-m) & =3 l
\end{aligned}
$$

em que $l$ é inteiro. Assim, para $\mathbf{k}=\left(\delta k_{x}, 4 \pi / 3 a+\delta k_{y}\right)$ teremos o seguinte vínculo imposto 
na função de onda:

$$
\delta \mathbf{k} \cdot \mathbf{C}_{h}=2 \pi q
$$

com $q$ sendo um número inteiro. Cada índice $q$ define uma linha de vetores $\mathbf{k}$ permitidos e cada linha contritui com uma banda $\pi$ oupada e uma banda $\pi^{*}$ desocupada. A Fig. 2.6 mostra a família de retas para um nanotubo $(5,5)$. Neste caso, as retas identificadas por $q \pm 5$ contribuem com auto-valores de energia que cruzam o nível de Fermi, o que configura um material metálico. A condição de que $(n-m=3 l)$ é sempre satisfeita por nanotubos armchair e para um subconjunto de nanotubos zigzag $(n, 0)$ quando $n$ for múltiplo de 3 . Um nanotubo será semicondutor quando a condição $(n-m=3 l \pm 1)$ for obedecida, de maneira que as bandas de $\pi$ e $\pi^{*}$ não se tocam no nível de Fermi resultando num gap de energia. Para mais detalhes, ver [41].

A Fig. 2.7 mostra as estruturas de bandas dos nanotubos de carbono ilustrados nas Fig. 2.5(a) e Fig. 2.5(b). Como discutido anteriormente, no nível de Fermi o nanotubo $(5,5)$ possui duas bandas de energia que se cruzam, resultando em "gap" de energia zero. Já para o nanotubo $(8,0)$, as bandas de valência e de condução não se cruzam no nível de Fermi e o sistema apresenta um "gap" de energia diferente de zero.

\subsection{Técnicas de crescimento de nanotubos de car- bono $(C N T s)$}

Existem três técnicas principais para crescimento de nanotubos de carbono: (i) descarga por arco-votáico; (ii) ablação a laser e (iii) deposição por vapor químico ( $C V D)$ [44]. A técnica de descarga por arco-voltáico foi a pioneira para o crescimento de CNTs. O aparato experimental utilizado por Iijima em 1991 [14] consistiu de uma câmara de vácuo com dois eletrodos de carbono, utilizados como fonte de carbono para os CNTs, e um gás inerte (hélio) utilizado para acelerar o processo de deposição de carbono. O processo ocorre quando é aplicada uma diferença de potencial alta entre os eletrodos de maneira a formar um plasma; os átomos de carbono são evaporados e depositados na superfície do anodo formando os CNTs, geralmente de paredes múltiplas ( $M W N T s$ ). Os nanotubos de parade única $(S W N T s)$ requerem um processo catalítico para promover seu crescimento, conforme publicado por Iijima em 1993 [42].

O método de ablação a laser consiste em esfoliar a superfície da amostra sólida de 


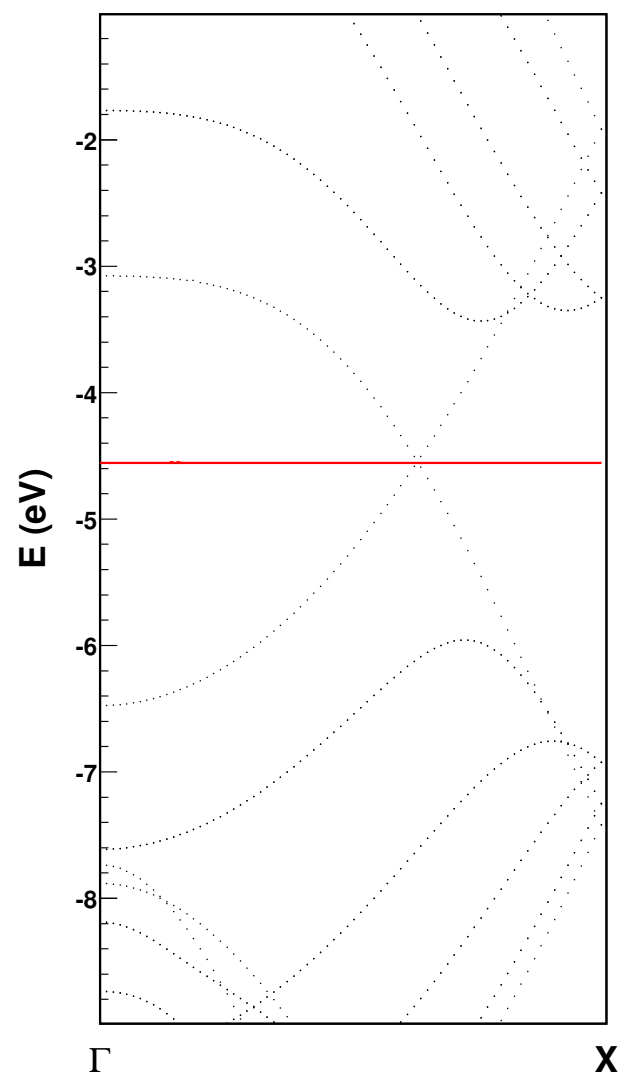

(a)

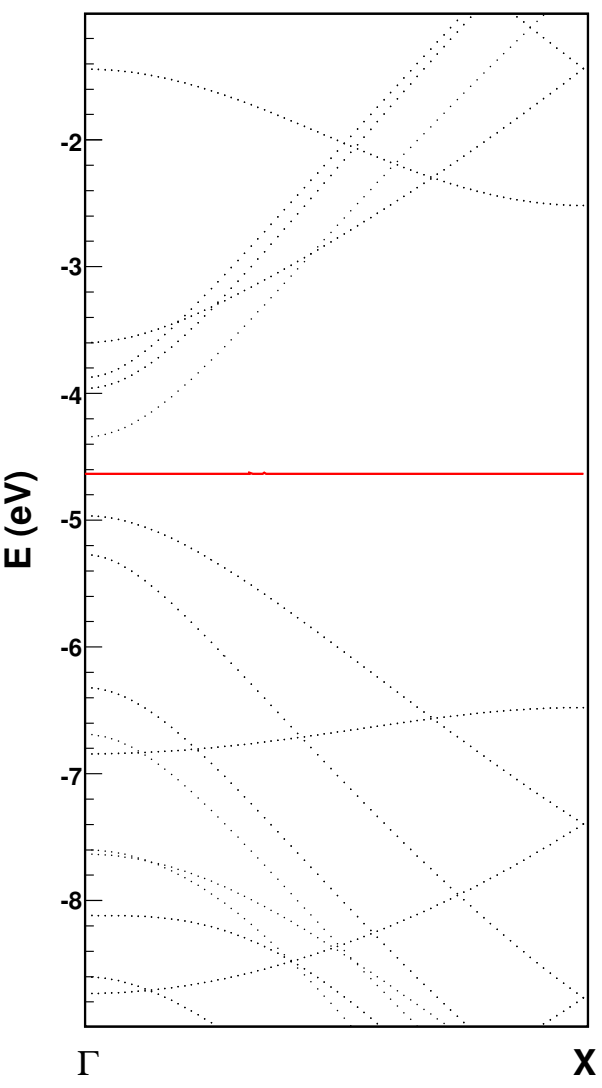

(b)

Figura 2.7: Estruturas de bandas: (a) nanotubo armchair (5,5) metálico;(b) nanotubo zigzag $(8,0)$ semicondutor.

carbono através de um feixe de laser intenso, que pode ser pulsado ou contínuo, em um forno a altas temperaturas preenchido com gás inerte. A amostra de grafite é geralmente misturada com pequenas quantidades de metais de transição que servem de catalizadores para o crescimento dos nanotubos. Essa técnica têm sido usada para produção de $S W N T s$ [45]. Em geral, a temperatura de crescimento dos nanotubos nessas duas técnicas, varia entre 3000-4000C ${ }^{\circ}$ já que a fonte de átomos de carbono é sólida.

Na técnica $C V D$, um gás de hidrocarbonetos (como por exemplo metano, acetileno ou etileno) é inserido numa câmara de reação. As moléculas de hidrocarbonetos são quebradas devido à temperatura elevada (em torno de 500-1000 $\mathrm{C}$ ) e essas espécies reagem com catalizadores (geralmente $\mathrm{Ni}, \mathrm{Fe}$ ou $\mathrm{Co}$ ) no substrato e os nanotubos são crescidos sobre esse substrato. Comparada com as duas técnicas anteriores, a técnica $C V D$ permite a síntese de nanotubos de carbono em temperaturas relativamente mais baixas. Com o controle dos parametros de catálise, pode-se crescer tanto MWNTs e SWNTs com 
alinhamento bastante controlado.

Em todas as técnicas mencionadas anteriormente, os nanotubos produzidos em geral apresentam defeitos, sejam eles estruturais ou até mesmo átomos contaminantes, o que pode produzir efeitos indesejáveis em suas propriedades. Desta forma, para a construção de dispositivos eficientes baseados em nanotubos de carbono é preciso investigar processos de purificação desses materiais. Geralmente, as impurezas mais comuns são alguns compostos de carbono que são subprodutos do processo de reação e algumas impurezas metálicas que são resíduos catalíticos. As impurezas constituintes de subprodutos de carbono podem ser removidas pelo aquecimento das amostras até atingir a temperatura de vaporização dessas impurezas. Já para remover as impurezas metálicas, em geral, os nanotubos são lavados com uma solução de ácido nítrico ou ácido sulfúrico e outros [46].

Com o desenvolvimento e modificação de diferentes processos de purificação, os nanotubos de carbono podem ser obtidos com pureza em torno de 99.6\% [47]. A qualidade estrutural e química dos nanotubos podem ser verificadas através de técnicas de microscopia tais como microscopia de força atômica $(A F M)$, microscopia de varredura e tunelamento $(S T M)$ e técnicas de espectrocopia tais como espectroscopia Raman.

As propriedades eletrônicas dos CNTs estão intimamente ligadas às propriedades estruturais (seção 2.3). As dificuldades experimentais de se obter nanotubos puros com quiralidade bem definida, ainda trazem problemas para a aplicação desses materias em nanodispositivos, devido à necessidade de se ter uma eletrônica bastante controlada. Nesse contexto, uma outra importante área que surge é o estudo dos processos de produção de nanotubos dopados com heteroátomos tais como nitrogênio e boro, o que permitiria um controle maior das propriedades eletrônicas do material através da composição química do mesmo e não especificamente pela estrutura geométrica [48].

A técnica $C V D$ descrita anteriomente tem sido uma das mais utilizadas para a produção de nanotubos dopados com heteroátomos. Entretanto, as tentativas de produção de nanotubos dopados com nitrogênio, por exemplo, mostram o crescimento de florestas de nanotubos parecidos com feixes de "bamboo" [32, 33]. Uma característica comum nesses experimentos é que as fontes de carbono (hidrocarbonetos) são misturadas com fontes de nitrogênio (acetonitrilas, piridinas, benzilaminas e etc) e esses compostos induzem desordem nas paredes dos nanotubos, o que parece limitar o crescimento de SWNTs. Entretanto, em um trabalho de 2006, Villalpando-Paez et al. [49] obtiveram SWNTs 
enriquecidos com nitrogênio $\left(C N_{x}\right)$ utilizando uma extensão da técnica $C V D$. Trabalhos recentes [50] mostram que é possível se ter um bom controle da concentração dos átomos dopantes, mas a distribuição destes defeitos ao longo do nanotubo ainda é desordenada. 


\section{Capítulo 3}

\section{Metodologia}

\subsection{Teoria do Funcional da Densidade - DFT}

Para a descrição das propriedades eletrônicas dos sistemas estudados nesse trabalho, utilizamos a Teoria do Funcional da Densidade (DFT). A descrição dos fundamentos da Teoria como os teoremas de Hohemberg-Kohn e as respectivas demonstrações; também o esquema proposto por Kohn-Sham para solução de problemas de muitos corpos baseado na DFT e as aproximações para os funcionais de troca e correlação, serão descritos no apêndice A deste trabalho. Decidimos por organizar o texto dessa maneira devido à DFT ser uma das teorias mais empregadas em estudos teóricos de sistemas de baixa dimensionalidade, de forma que acreditamos ser um assunto bastante conhecido e abordado pela grande maioria das dissertações e teses na área.

Os cálculos de DFT serão feitos com o programa SIESTA [37]. Ele utilizada conjunto de base localizadas e a teoria de pseudo potenciais ${ }^{1}$ para descrever os elétrons de "caroço ". Isso permite a descrição de sistemas maiores com centenas e até milhares de átomos. Além disso, o fato de se usar orbitais localizados o torna bastante adequado para ser acoplado com a teoria quântica de transporte em termos de funções de Green, como enfatizaremos ao abordar esse formalismo.

Para efetuarmos os cálculos de estrutura eletrônica dos sistemas pretendidos nesse trabalho, fizemos um estudo prévio da influência de alguns parâmetros exigidos pelo programa SIESTA sobre a energia total do nanotubo $(5,5)$, afim de determinar quais os valores ótimos destes parâmetros para a realização das simulações. Os valores dos parâmetros

\footnotetext{
${ }^{1}$ Ver capítulo 7 da referência [51].
} 
utilizados nos cálculos, dados relacionados ao conjunto de funções base, aproximações para o termo de troca e correlação dentro da DFT e outras informações relevantes para a simulação, estão descritas no apêndince B.

\subsection{Teoria quântica de Transporte eletrônico}

Quando nos referimos a transporte eletrônico em um dispositivo, estamos interessados em estudar o comportamento de algum material ligado a eletrodos, aos quais aplicamos um potencial externo, por exemplo o criado por uma bateria (Fig. 3.1). Nas últimas décadas

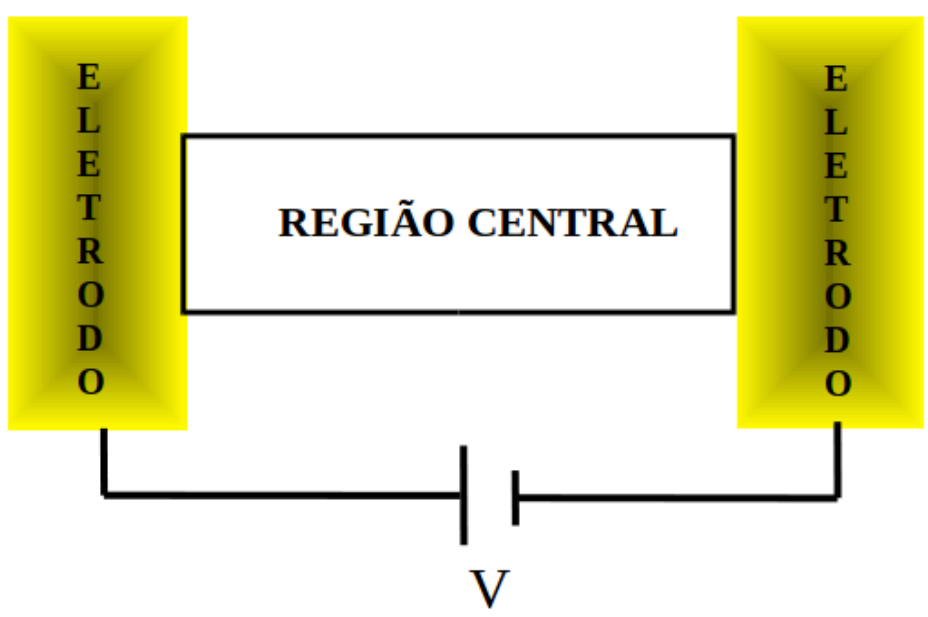

Figura 3.1: Esquema pictórico de um dispositivo eletrônico constituído de uma região central na qual desejamos investigar as propriedades de transporte de elétrons e os eletrodos. Todo sistema é submetido a um potencial externo, por exemplo, o criado por uma bateria.

ocorreram avanços importantes na área de semicondutores que possibilitou a fabricação de dispositivos cada vez menores em que efeitos quânticos, como por exemplo a quantização da condutância e efeito Hall quântico, podem ser observados diretamente por experimentos de transporte eletrônico. Com a miniaturização, alguns fatores limitantes surgiram como o da potência dissipada e tunelamento quântico que podem diminuir a eficiência dos dispositivos lógicos. Desta forma, para se avançar ainda mais na miniaturização de forma a construir dispositivos microscópicos, era preciso entender a influência desses efeitos.

Os primeiros passos no desenvolvimento de uma teoria de transporte que pudesse descrever o transporte eletrônico em sistemas nanométricos foram dados por R. Landauer [52]. Outras contribuições importantes para o desenvolvimento de um formalismo de 
transporte eletrônico quântico foi dada por Büttiker $[53,54]$ que estendeu as idéias de Landauer para sistemas de vários contatos, o qual ficou conhecido como formalismo de Landauer-Büttiker.

\subsubsection{Quantização da Condutância}

Nessa seção discutiremos a aproximação de Landauer, a qual tem sido amplamente utilizada para tratar transporte de elétrons nesses sistemas e como surge a quantização da condutância em dispositivos microscópicos. Nessa aproximação, a corrente através de um condutor (o qual corresponde à região central da Fig. 3.1) é expressa em termos da probabilidade que um elétron tem de ser transmitido através do condutor. Landauer [55] relacionou a resposta linear da condutância à probabilidade de transmissão através de um condutor e discutiu questões fundamentais que surgem quando aplicamos essa relação a condutores que apresentam probabilidade de transmissão próxima da unidade. Por exemplo, se aplicarmos uma diferença de potencial (ddp) entre dois eletrodos ligados a um condutor balístico ${ }^{2}$, a corrente é finita indicando que a resistência é diferente de zero. Mas pode um condutor balístico ter resistência? Se não, de onde vem essa resistência? Essas questões serão respondidas de maneira simples e serão apresentadas as mudanças que surgem ao tratar a condutância de sistemas nanoscópicos.

Considere uma região central de seção reta $W$ e comprimento $l$ ligado a dois eletrodos. Se as dimensões da região central forem da ordem das dimensões dos eletrodos (da ordem de micrometros), a condutância é dada por $G=\sigma W / l$ (lei de Ohm), em que a condutividade $\sigma$ é uma propriedade do material. À medida que reduzimos o comprimento $l$, se o comportamento ôhmico fosse obedecido, esperaríamos que a condutância aumentasse indefinidamente. Entretanto, experimentalmente, observa-se que a condutância tende a um valor limite quando o dispositivo é muito menor que o livre caminho médio do elétron. Essa resistência surge na interface entre os eletrodos e o dispositivo. Nos eletrodos existem infinitas sub-bandas ou modos normais que contribuem para a corrente, enquanto na região central existem poucas (supondo que $W$ é pequeno quando comparado às dimensões dos eletrodos). Isso requer uma redistribuição da corrente dos canais na interface entre os eletrodos e a região central, e é essa redistribuição a responsável pela resistência

\footnotetext{
${ }^{2}$ Condutor balístico: o livre caminho médio do elétron é muito maior que o comprimento do condutor. Para um condutor balístico ideal a probabilidade de transmissão é igual a 1.
} 
observada.

Consideramos nessa aproximação que os eletrodos são metálicos de forma que eles não são perturbados pela saída ou entrada de elétrons. Assim, pensamos nos eletrodos como reservatórios de elétrons e que a descrição dos mesmos possa ser feita pelos respectivos potenciais químicos $\mu$. Para simplificar a apresentação das idéias principais relacionadas à aproximação de Landauer, supomos temperatura igual a zero, de maneira que só há fluxo de corrente no intervalo de energia $\mu_{L}>E>\mu_{R}$, em que $\mu_{L(R)}$ é o potencial químico do eletrodo esquerdo(direito).

Para cada sub-banda na região central temos a relação de dispersão $E(N, k)$, em que $N$ é o índice da sub-banda e $k$ é o vetor de onda, conforme mostrado na Fig. 3.2. Definindo uma energia de corte $\epsilon_{N}=E(N, k=0)$ para cada sub-banda $N$, em que abaixo

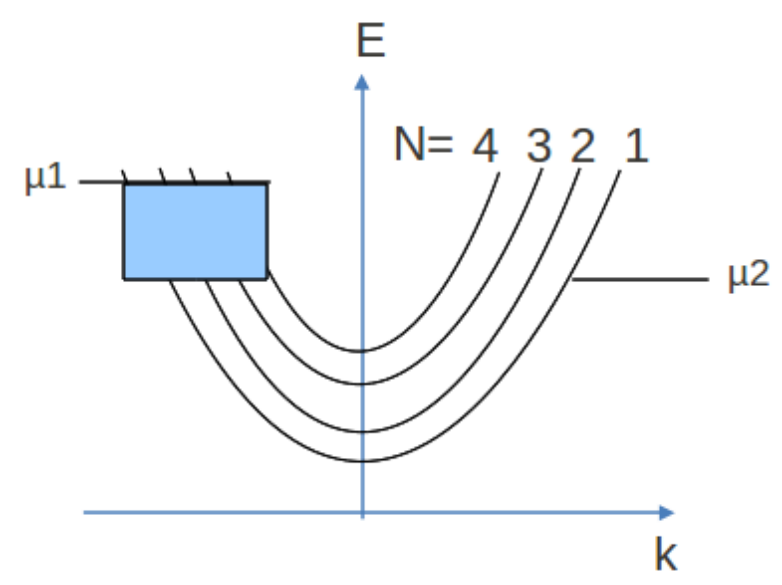

Figura 3.2: Esquema pictórico das relações de dispersão para diferentes sub-bandas da região central do dispositivo. O retângulo colorido mostra a região de energia dos elétrons que poderiam resultar em corrente elétrica.

dessa energia elétrons não podem se propagar, o número de sub-bandas $M(E)$ em uma determinada energia $E$ é obtido contando o número de sub-bandas que possuem energia de corte menor que $E$ :

$$
M(E)=\sum_{N} \vartheta\left(E-\epsilon_{N}\right),
$$

em que $\vartheta$ é a função de Heaviside com

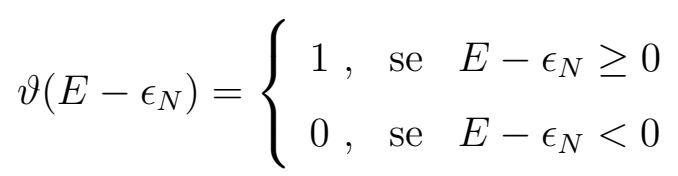


Consideremos primeiramente, uma única sub-banda de energia em que os estados $k$ são ocupados segundo à função de distribuição de Fermi-Dirac,

$$
f\left(E, \mu_{L, R}\right)=\frac{1}{e^{-\frac{E-\mu_{L, R}}{K_{B}}}+1},
$$

em que $\Theta$ é a temperatura. Um gás de elétrons uniforme com $n$ elétrons por unidade de comprimento que se movem com velocidade igual a $v$ corresponde a uma corrente igual a $I=e n v$. Desta forma a corrente através dos estados $k$ é dada por

$$
I=\frac{e}{l} \sum_{k} v f(E)=\frac{e}{l} \sum_{k} \frac{1}{\hbar} \frac{\partial E}{\partial k} f(E) .
$$

Supondo condições de contorno periódicas para as soluções da Equação de Schröedinger, temos a seguinte correspondência

$$
\sum_{k} \rightarrow 2(\operatorname{spin}) \times \frac{l}{2 \pi} \int d k,
$$

o que nos permite escrever (fazendo uma mudança de variáveis)

$$
I=\frac{2 e}{h} \int_{-\infty}^{\infty} f(E) d E
$$

A corrente devido às várias sub-bandas no intervalo de energia é calculada somando sobre todas as sub-bandas $M(E)$,

$$
I=\frac{2 e}{h} \int_{-\infty}^{\infty} M(E) f(E) d E .
$$

Supondo que o número de sub-bandas $M$ é independente da energia no intervalo $\mu_{L}>E>\mu_{R}$, podemos escrever $(f(E)=1$ em $\Theta=0 K)$

$$
\begin{aligned}
& I=\frac{2 e^{2}}{h} M \frac{\left(\mu_{L}-\mu_{R}\right)}{e} \quad \Longrightarrow \quad G_{c}=\frac{2 e^{2}}{h} M \\
& G_{c}^{-1} \equiv \frac{\left(\mu_{L}-\mu_{R}\right) / e}{I} \quad \Longrightarrow \quad G_{c}^{-1} \approx \frac{12.8 k \Omega}{M},
\end{aligned}
$$

em que $G_{c}^{-1}$ é a resistência entre o eletrodo e a região central e $\left(\mu_{L}-\mu_{R}\right) / e$ é o potencial externo aplicado nos eletrodos esquerdo e direito. Como estamos considerando eletrodos metálicos, o potencial aplicado aos eletrodos serve para modificar os potenciais químicos dos eletrodos esquerdo e direito por:

$$
\mu_{L}=\mu_{0}+\frac{e V}{2}
$$

e

$$
\mu_{R}=\mu_{0}-\frac{e V}{2}
$$


respectivamente, em que $\mu_{0}$ é o potencial químico na situação de equilíbrio.

Toda discussão até agora foi feita para um dispositivo balístico ideal com probabilidade de transmissão igual a 1. Para um dispositivo balístico qualquer, devemos incluir um fator multiplicativo T na Eq. 3.8 que é a probabilidade média de transmissão que um elétron vindo de um eletrodo, por exemplo o esquerdo, tem de atravessar a região central e chegar ao eletrodo direito. Desta forma, a Eq. 3.8 pode ser reescrita como ${ }^{3}$

$$
G_{c}=\frac{2 e^{2}}{h} M T
$$

que é conhecida como relação de Fischer-Lee [56]. Deve-se observar a quantização da condutância em unidades da constante $G_{0} \equiv 2 e^{2} / h$, uma vez que $G_{c}$ depende discretamente do número de sub-bandas $M$.

Resumindo, sistemas pertencentes à escala macroscópica obedecem à $G=\sigma W / l$. Entretanto quando vamos para a escala nanométrica, algumas correções são necessárias: (1) Existe uma resistência na interface entre o eletrodo e a região central que é independente do comprimento $l$ da mesma. (2) A condutância não diminui linearmente com a largura $W$ da região central mas depende discretamente do número de sub-bandas desta. (3) A condutância depende linearmente da probabilidade de transmissão $T$ através do dispositivo.

Com a temperatura $0 K$, elétrons vindo do eletrodo esquerdo $\left(\mu_{L}\right)$ podem ser transmitidos através do dispositivo e ocuparem estados no eletrodo direito $\left(\mu_{R}\right)$, desde que tenham energia entre $\mu_{L}$ e $\mu_{R}$. Entretanto, elétrons vindo do eletrodo direito não podem ser transmitidos através do dispositivo e ocuparem estados no eletrodo esquerdo, uma vez que seus estados estão todos ocupados devido à distribuição de Fermi-Dirac em $0 K$, como mostrado na Fig. 3.3(a).

Entretanto, para o caso de temperatura diferente de zero, os elétrons que podem ser transmitidos não são apenas aqueles com energia entre os potenciais químicos dos eletrodos depois da aplicação do potencial externo. Devemos levar em conta as distribuições de Fermi-Dirac para os elétrons em cada eletrodo, conforme Fig. 3.3(b). Assim, fazemos uma correção na Eq. 3.7 de forma que a corrente resultante através do dispositivo para

\footnotetext{
${ }^{3}$ Aqui consideramos que a probabilidade de transmissão é igual para todas as sub-bandas. No caso de probabilidades diferentes, podemos generalizar a equação tomando $M T=\sum_{i=1}^{M} T_{i}$.
} 


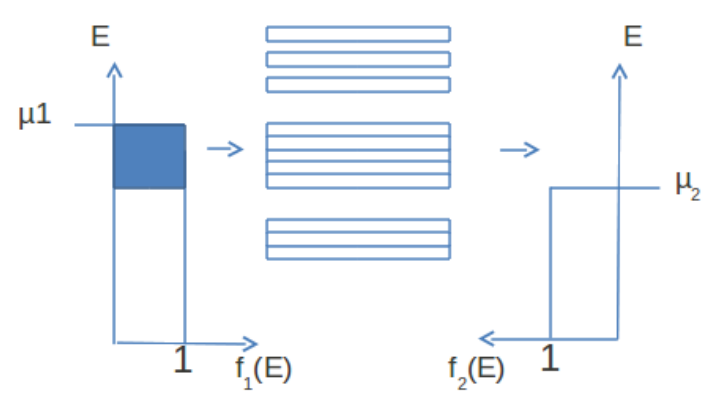

(a)

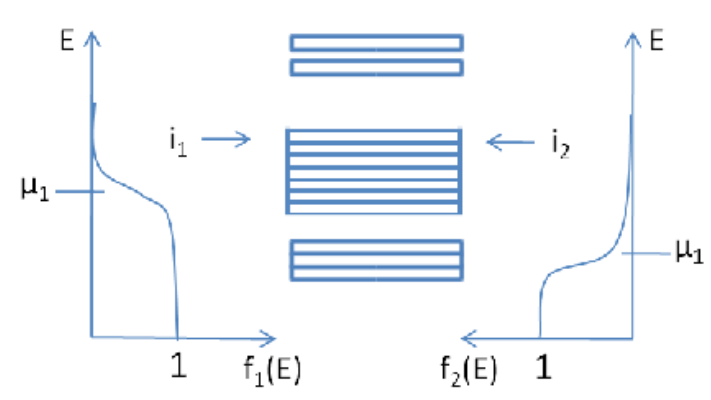

(b)

Figura 3.3: (a)Função distribuição dos elétrons incidentes em temperatura 0K. A região colorida representa o intervalo de energias possíveis para os elétrons vindo do eletrodo esquerdo. (b) Função distribuição dos elétrons em temperatura diferente de zero.

temperatura diferente de zero é dada portanto

$$
I=\frac{2 e}{h} \int_{-\infty}^{+\infty} \tilde{T}(E)\left(f_{L}(E)-f_{R}(E)\right) d E
$$

em que $\tilde{T}(E)=M(E) T(E)$, em que consideramos a soma sobre toda a energia, já que na realidade as sub-bandas são dependentes da energia.

\section{Regime linear}

Na situação de equilíbrio em que não há potencial externo aplicado, temos que $I=0$ quando

$$
\begin{cases}\mu_{L} \rightarrow \mu_{R}, & \text { para } 0 K \\ f_{L}(E) \rightarrow f_{R}(E), & \text { para outras temperaturas. }\end{cases}
$$

Para pequenas flutuações em torno do equilíbrio, a corrente é proporcional ao potencial externo aplicado.

$$
\left.d I=\frac{2 e}{h} \int\{[\tilde{T}(E)]_{e q} d\left[\left(f_{L}(E)-f_{R}(E)\right)\right]+\underbrace{0}_{\left[\left(f_{L}(E)-f_{R}(E)\right]_{e q}\right.} d[\tilde{T}(E))_{e q}]\right\}
$$

Expandindo o primeiro termo da Eq. 3.13 em séries de Taylor, obtemos

$$
\begin{gathered}
d f_{\mu_{i}} \approx\left(\frac{\partial f_{\mu_{i}}}{\partial \mu_{i}}\right)_{\mu_{i}=\mu_{0}} d \mu_{i}, \quad \text { com } d \mu_{i}=\mu_{i}-\mu_{0} \\
d\left[f_{L}-f_{R}\right] \approx\left(\frac{\partial f_{\mu}}{\partial \mu}\right)_{\mu=\mu_{e q}}\left[\mu_{L}-\mu_{R}\right]=\left(-\frac{\partial f_{0}}{\partial E}\right)_{\mu=\mu_{e q}}\left[\mu_{L}-\mu_{R}\right]
\end{gathered}
$$


onde $f_{0}$ é a função de Fermi-Dirac no equilíbrio $\left(\mu=E_{f}\right)$. Desta forma podemos escrever:

$$
G=\frac{d I}{\left(\mu_{L}-\mu_{R}\right) / e}=\frac{2 e^{2}}{h} \int \tilde{T}(E)\left(-\frac{\partial f_{0}}{\partial E}\right) d E .
$$

Para baixas temperaturas, temos a seguinte correspondência

$$
\begin{gathered}
f_{0} \approx \vartheta\left(E_{f}-E\right) \quad \Longrightarrow \quad-\frac{\partial f_{0}}{\partial E} \approx \delta\left(E_{f}-E\right) \\
\vartheta\left(E_{f}-E\right)=\left\{\begin{array}{ll}
1, & \text { se } E_{f}-E \geq 0 \\
0, & \text { se } E_{f}-E<0
\end{array} \quad, \quad \delta\left(E_{f}-E\right)=\left\{\begin{array}{lll}
1, & \text { se } & E_{f}=E \\
0, & \text { se } & E_{f} \neq E
\end{array}\right.\right.
\end{gathered}
$$

em que $\vartheta\left(E_{f}-E\right)$ e $\delta\left(E_{f}-E\right)$ são as funções de Heaviside e delta de Dirac, respectivamente.

Finalmente, substituindo a Eq. 3.16 na Eq. 3.15, chegamos à fórmula de regime linear dada por

$$
G=\frac{2 e^{2}}{h} \int \tilde{T}(E) \delta\left(E_{f}-E\right) d E \quad \Longrightarrow \quad G=\frac{2 e^{2}}{h} \tilde{T}\left(E_{f}\right) .
$$

Uma condição suficiente para a validade da aproximação de regime linear é quando o potencial externo aplicado $\left(\mu_{L}-\mu_{R}\right)$ é muito menor que $k_{B} \Theta$, o que garante que a aproximação de $1^{0}$ ordem da série de Taylor (Eq. 3.14) possa ser aplicada.

\subsubsection{Formalismo da Função de Green Fora do Equilíbrio (NEGF)}

O tipo de sistema para o qual desejamos investigar as propriedades de transporte consiste de uma região central que chamamos de região de espalhamento, a qual é ligada a dois eletrodos nas duas extremidades, conforme mostrado na Fig. 3.4. Nesse tipo de sistemas, onde a região de espalhamento bem como os eletrodos têm dimensões atômicas, o sistema como um todo configura um sistema quântico com condições de contorno abertas, ou seja, o sistema é infinito e sem periodicidade.

A descrição do problema de transporte eletrônico pode ser vista por três perspectivas distintas: do ponto de vista termodinâmico, eletrostático e de mecânica quântica ${ }^{4}$. Do ponto de vista termodinâmico, os eletrodos esquerdo(L) e direito(R) são entendidos como dois reservatórios de elétrons em equilíbrio termodinâmico e são descritos pelos respectivos potenciais químicos $\mu_{L, R}$. Quando o sistema está em equilíbrio termodinâmico, todo

\footnotetext{
${ }^{4} \mathrm{~A}$ apresentação que seguiremos para essa parte da metodologia está detalhada nas referencias [57, 58].
} 


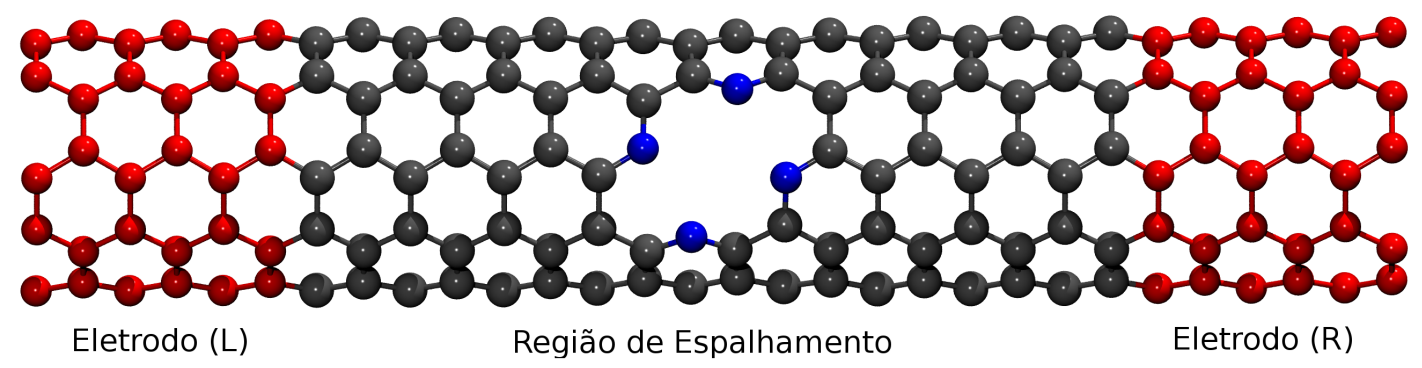

Figura 3.4: Tipo de sistema que desejamos investigar as propriedades de transporte eletrônico. Região central de espalhamento e os eletrodos esquerdo (L) e direito (R).

o sistema (região de espalhamento e eletrodos) estão em um mesmo potencial químico. Se uma fonte externa de potencial é aplicada ao sistema, ocorrerá o desnivelamento dos potenciais químicos e consequentemente transferências de carga entre os eletrodos e uma corrente elétrica será estabelecida. Isso ocorre pois o sistema tenta retornar ao equilíbrio termodinâmico. Ao mantermos fixa a diferença entre os potenciais (simulando uma bateria) obteremos um estado estacionário.

Quando o sistema é submetido a uma diferença de potencial, haverá uma redistribuição de carga no sistema. Pelo fato de os eletrodos serem bons condutores, supõe-se que a queda de potencial ocorra apenas na região de espalhamento, que deve ser resolvida autoconsistentemente, levando em conta a redistribuição de cargas. Por outro lado, a mudança nos eletrodos é dada pela variação por um constante nos potenciais químicos, de acordo com as Eqs. 3.9 e 3.10 .

Da ótica da mecânica quântica, o sistema pode ser dividido em três regiões: eletrodos esquerdo e direito e a região de espalhamento. Entende-se por região de espalhamento qualquer estrutura que quebre a periodicidade dos eletrodos. Podem ser: defeitos, uma molécula, nanofios, nanoestruturas de carbono etc; no presente caso, é o defeito NR. Os eletrodos podem ser vistos como cadeias semi-infinitas e periódicas na direção de transporte. Cada unidade dessas cadeias é chamada de camada principal $(C P)$ e é descrita por um hamiltoniano $H_{0}$. Esse operador é representado por uma matriz que depende das dimensões da célula unitária do sistema. Utilizando conjunto base de funções localizadas nos átomos, cada camada principal é escolhida para ter apenas interação com a camada adjacente. Desta forma, a dimensão da matriz que representa o operador $H_{0}$ será $N \times N$, em que $N=\sum_{i}^{N_{\text {espécies }}} N_{\text {átomos }} \times N_{\text {orbitais }}$ é o número de graus de liberdade de cada $C P$. 
Como o programa SIESTA usa esse tipo de funções base, ele é atrativo para esse tipo de abordagem. O operador $H_{1}$ descreve o acoplamento entre as $C P s$ adjacentes, e o operador $H_{D}$ descreve a região de espalhamento. Por fim, o acoplamento entre a região de espalhamento e o eletrodo esquerdo(direito) é dado por $H_{L D}\left(H_{D R}\right)$.

O hamiltoniano do sistema completo pode ser escrito pela matriz:

$$
\mathcal{H}=\left(\begin{array}{cccccccccc}
. & . & . & . & . & . & . & . & . & . \\
. & 0 & H_{-1} & H_{0} & H_{1} & 0 & . & . & . & . \\
\cdot & . & 0 & H_{-1} & H_{0} & H_{L D} & 0 & . & . & . \\
. & . & . & 0 & H_{D L} & H_{D} & H_{D R} & 0 & . & . \\
\cdot & . & . & . & 0 & H_{R D} & H_{0} & H_{1} & . & . \\
. & . & . & . & 0 & H_{-1} & H_{0} & H_{1} & . \\
. & . & . & . & . & . & . & . & .
\end{array}\right)
$$

em que $H_{-1}=H_{1}^{\dagger}, H_{D L}=H_{L D}^{\dagger}$ e $H_{D R}=H_{R D}^{\dagger}$. Esse operador é infinito e não periódico, uma vez que a periodicidade foi quebrada devido à presença do defeito entre os eletrodos. A solução do problema seria, em princípio, diagonalizar essa matriz que é infinita.

O Formalismo das Funções de Green Fora do Equilíbrio (NEGF) é uma maneira alternativa para tratar o problema de transporte quântico [59]. Dentro desse formalismo, a equação de Schröedinger pode ser escrita em termos de funções de Green por:

$$
\left[\epsilon^{+} S-\mathcal{H}\right] \mathcal{G}=\mathcal{I}
$$

em que $\epsilon^{+}=\lim _{\delta \rightarrow 0^{+}} E+i \delta$ é a energia e $S$ é a matriz "overlap" que aparece devido à não ortogonalidade das funções base. De forma matricial, a Eq. 3.19 é escrita por:

$$
\left(\begin{array}{ccc}
\epsilon^{+} S_{L}-\mathcal{H}_{L} & \epsilon^{+} S_{L D}-\mathcal{H}_{L D} & 0 \\
\epsilon^{+} S_{D L}-\mathcal{H}_{D L} & \epsilon^{+} S_{D}-H_{D} & \epsilon^{+} S_{D R}-\mathcal{H}_{D R} \\
0 & \epsilon^{+} S_{R D}-\mathcal{H}_{R D} & \epsilon^{+} S_{R}-\mathcal{H}_{R}
\end{array}\right)\left(\begin{array}{ccc}
\mathcal{G}_{L} & \mathcal{G}_{L D} & \mathcal{G}_{L R} \\
\mathcal{G}_{D L} & G_{D} & \mathcal{G}_{D R} \\
\mathcal{G}_{R L} & \mathcal{G}_{R D} & \mathcal{G}_{R}
\end{array}\right)=\left(\begin{array}{ccc}
\mathcal{I} & 0 & 0 \\
0 & \mathcal{I} & 0 \\
0 & 0 & \mathcal{I}
\end{array}\right)
$$

em que as matrizes $\mathcal{H}_{L}, \mathcal{H}_{R}, \mathcal{H}_{L D}$ e $\mathcal{H}_{D R}$ são:

$$
\mathcal{H}_{L}=\left(\begin{array}{ccccc}
\ddots & \ddots & \ddots & \ddots & \vdots \\
0 & H_{-1} & H_{0} & H_{1} & 0 \\
\cdots & 0 & H_{-1} & H_{0} & H_{1} \\
\cdots & \cdots & 0 & H_{-1} & H_{0}
\end{array}\right),
$$




$$
\begin{gathered}
\mathcal{H}_{R}=\left(\begin{array}{ccccc}
H_{0} & H_{1} & 0 & \ldots & \ldots \\
H_{-1} & H_{0} & H_{1} & 0 & \ldots \\
0 & H_{-1} & H_{0} & H_{1} & 0 \\
\vdots & \ddots & \ddots & \ddots & \ddots
\end{array}\right) \\
\mathcal{H}_{R}=\left(\begin{array}{c}
\vdots \\
0 \\
H_{L D}
\end{array}\right)
\end{gathered}
$$

$\mathrm{e}$

$$
\mathcal{H}_{D R}=\left(\begin{array}{lll}
H_{R D} & 0 & \cdots
\end{array}\right) .
$$

A Eq. 3.20 pode ser resolvida fazendo o produto matricial convencional, o que resulta em 9 equações. Entretanto, se supusermos que os eletrodos são metálicos, o acoplamento do dispositivo a esses não deve influenciar a estrutura eletrônica dos mesmos. Isso permitenos focar apenas na região do dispositivo e resolver explicitamente 3 equações. Assim, a partir da Eq. 3.19, a função de Green da parte central pode ser determinada resolvendo as seguintes equações:

$$
\begin{aligned}
\left(\epsilon^{+} S_{L}-\mathcal{H}_{L}\right) \mathcal{G}_{L D}+\left(\epsilon^{+} S_{L D}-\mathcal{H}_{L D}\right) G_{D} & =0 \\
\left(\epsilon^{+} S_{D L}-\mathcal{H}_{D L}\right) \mathcal{G}_{L D}+\left(\epsilon^{+} S_{D}-H_{D}\right) G_{D}+\left(\epsilon^{+} S_{D R}-\mathcal{H}_{D R}\right) \mathcal{G}_{R D} & =\mathcal{I} \\
\left(\epsilon^{+} S_{R D}-\mathcal{H}_{R D}\right) \mathcal{G}_{L D}+\left(\epsilon^{+} S_{R}-\mathcal{H}_{R}\right) \mathcal{G}_{R D} & =0
\end{aligned}
$$

em que fizemos o produto das 3 linhas da primeira matriz pela $2^{\underline{O}}$ coluna da $2^{\underline{O}}$ matriz. Isolando $\mathcal{G}_{L D}$ e $\mathcal{G}_{R D}$ nas Eq. 3.25 e Eq. 3.27, respectivamente, temos:

$$
\begin{gathered}
\mathcal{G}_{L D}=\left(\epsilon^{+} S_{L}-\mathcal{H}_{L}\right)^{-1}\left(\mathcal{H}_{L D}-\epsilon^{+} S_{L D}\right) G_{D} \\
\mathcal{G}_{R D}=\left(\epsilon^{+} S_{R}-\mathcal{H}_{R}\right)^{-1}\left(\mathcal{H}_{R D}-\epsilon^{+} S_{R D}\right) G_{D} .
\end{gathered}
$$

Definindo as funções de Green de superfície $g_{L}=\left(\epsilon^{+} S_{L}-\mathcal{H}_{L}\right)^{-1}$ e $g_{R}=\left(\epsilon^{+} S_{R}-\mathcal{H}_{R}\right)^{-1}$, que estão relacionadas com as $C P s$ adjacentes à região do dispositivo, podemos reescrever as Eq. $\quad 3.28$ e Eq. 3.29 por:

$$
\begin{gathered}
\mathcal{G}_{L D}=g_{L}\left(\mathcal{H}_{L D}-\epsilon^{+} S_{L D}\right) G_{D} \\
\mathcal{G}_{R D}=g_{R}\left(\mathcal{H}_{R D}-\epsilon^{+} S_{R D}\right) G_{D} .
\end{gathered}
$$

Substituindo as Eq. 3.30 e Eq. 3.31 em Eq. 3.26 e resolvendo $G_{D}$, temos:

$$
G_{D}=\left[\left(\epsilon^{+} S_{D}-H_{D}\right)-\Sigma_{L}-\Sigma_{R}\right]^{-1},
$$


em que $\Sigma_{L}$ e $\Sigma_{R}$ são dadas por,

$$
\begin{gathered}
\Sigma_{L}=\left(\mathcal{H}_{L D}-\epsilon^{+} S_{L D}\right) g_{L}\left(\mathcal{H}_{D L}-\epsilon^{+} S_{D L}\right) \\
\Sigma_{R}=\left(\mathcal{H}_{R D}-\epsilon^{+} S_{R D}\right) g_{R}\left(\mathcal{H}_{D R}-\epsilon^{+} S_{D R}\right) .
\end{gathered}
$$

Essas quantidades são as auto-energias dos eletrodos esquerdo(L) e direito(R) e descrevem os efeitos causados por estes na região de espalhamento. As quantidades que descrevem as taxas de transição dos elétrons entrarem ou saírem da região de espalhamento são:

$$
\Gamma_{L, R}=i\left(\Sigma_{L, R}-\Sigma_{L, R}^{\dagger}\right)
$$

Considerando as funções de Green retardadas $\left(G_{D}\right)$ e avançadas $\left(G_{D}^{\dagger}\right)$ para a solução do problema de transporte (ver capítulo 3 da referência [60]), a transmitância pode ser calculada por:

$$
T=\operatorname{Tr}\left[\Gamma_{L} G_{D}^{\dagger} \Gamma_{R} G_{D}\right]
$$

Finalmente, fazendo uso da Eq. 3.37 e da discussão da seção 3.2.1, a corrente elétrica é calculada por:

$$
I=\frac{2 e}{h} \int_{-\infty}^{+\infty} \sum_{\sigma=\uparrow, \downarrow} \operatorname{Tr}\left[\Gamma_{L}^{\sigma} G_{D}^{\dagger \sigma} \Gamma_{R}^{\sigma} G_{D}^{\sigma}\right]\left[f_{L}\left(E, \mu_{L}\right)-f_{R}\left(E, \mu_{R}\right)\right] d E,
$$

em que consideramos a transmitância em diferentes estados de $\operatorname{spin}(u p=\uparrow$ e down $=\downarrow)$.

Para resolver o problema de transporte eletrônico, unimos o formalismo (NEGF) com cálculos de estrutura eletrônica. Dentro do formalismo da DFT em que o hamiltoniano do sistema é tido como funcional da densidade eletrônica $\mathcal{H}[n]$, pode-se resolver o problema sujeito a um potencial externo aplicado através de um processo auto-consistente $[57,58]$. Em princípio, o formalismo descrito acima, poderia ser usado para calcular as propriedades de transporte de qualquer sistema unidimensional, desde que o hamiltoniano $\mathcal{H}$ seja conhecido em função da densidade eletrônica.

Ainda através do formalismo das funções de Green, pode-se determinar grandezas físicas como a densidade de estados (DOS) e a densidade de estados projetada (PDOS) do sistema aberto. Pode-se focar na função de Green da região central, que leva em conta o efeito dos eletrodos através das auto-energias, e definir a DOS como:

$$
\operatorname{DOS}(E)=\frac{1}{2 \pi} \operatorname{Tr}\left[\operatorname{Im}_{D}(E) S_{D}\right]
$$


Analogamente, pode-se definir a PDOS sobre os orbitais da base. Como o formalismo das funções de Green foi construído sobre uma base de orbitais atômicos, os termos da projeção correspondem aos elementos da diagonal da Eq. 3.39:

$$
\operatorname{PDOS}(E)=\frac{1}{2 \pi} \operatorname{Im}\left[G_{D}(E) S_{D}\right]_{\mu \mu}
$$

Os métodos convencionais de DFT (por exemplo o SIESTA) considera condições periódicas de contorno para solução do problema eletrônico, ou seja, no caso do nanotubo com defeito, esse defeito é periodicamente espaçado. Entretanto, o sistema real para o qual desejamos investigar as propriedades de transporte, é um nanotubo infinito com um único defeito (ou um número finito deles aleatoriamente distribuídos). Em um sistema metálico, a presença de um potencial periódico fraco faz surgir uma banda de defeito que por sua vez pode gerar anti-cruzamentos na estrutura de bandas e, consequentemente, o surgimento de mini-gaps. Desta forma, como o formalismo das funções de Green trata o sistema aberto, o cálculo da DOS e PDOS pelas Eq. 3.39 e Eq. 3.40, respectivamente, elimina esse problema [61].

\subsubsection{Transporte eletrônico em sistemas desordenados}

Quando estamos tratando de sensores de gás de nanotubos de carbono, devemos ter em mente que esses nanotubos podem ter centenas de nanometros de comprimento, contendo dezenas de milhares de átomos. Resolver um sistema destas dimensões considerando cálculos atomísticos ainda é impraticável computacionalmente. A Fig. 3.5(a) mostra um trecho de um nanotubo contendo defeitos aleatoriamente distribuídos. Obviamente, os sistemas que desejamos simular são de aproximadamente $\sim 200 \mathrm{~nm}$ e 20000 átomos. Pela metodologia apresentada na seção 3.2.2 sabemos como encontrar a função de Green da região de espalhamento (Fig. 3.4). Gostaríamos de solucionar o problema de transporte considerando que a região de espalhamento agora seja um sistema conforme o mostrado na Fig. 3.5(a), então precisamos encontrar a função de Green do mesmo. Como consequência do sistema ser longo e unidimensional, supomos que este seja constituído de vários blocos, Fig. 3.6(b). Cada bloco é descrito por um hamiltoniano $H_{i}$ e o acoplamento entre um bloco com seu vizinho mais próximo é dado por $V_{i j}=V_{i j} \delta_{i j \pm 1}$, conforme esquema da Fig. 3.6.

Semelhantemente ao que foi feito para o caso da região de espalhamento conectada a 
(a)

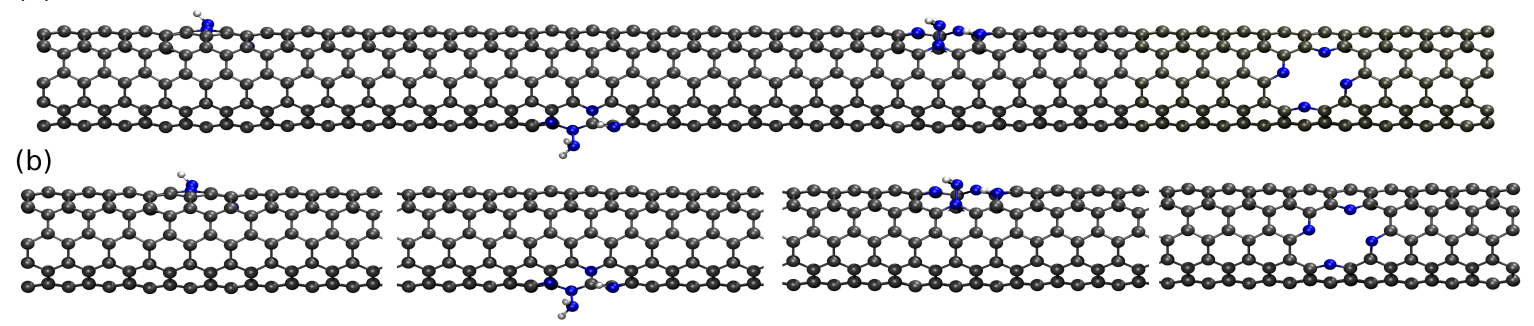

Figura 3.5: (a) Segmento de nantotubo com defeitos aleatoriamente distribuídos (região de espalhamento). O nanotubo apresentado tem $\sim 9 \mathrm{~nm}$ de comprimento. Os nanotubos realmente utilizados nos nossos trabalhos tem $\sim 220 \mathrm{~nm}$. (b) Blocos de defeitos que constituem o nanotubo.

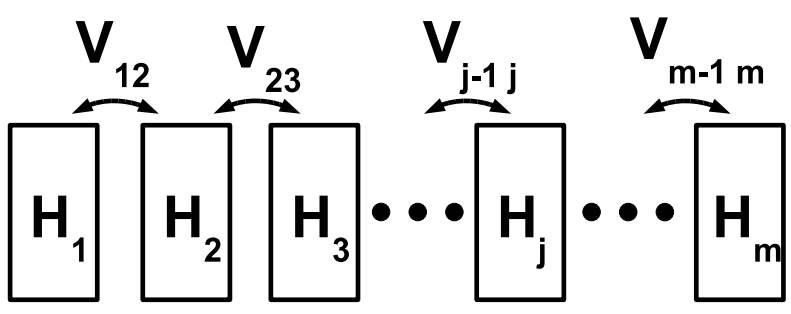

Figura 3.6: Esquema mostrando uma região de espalhamento constituída de m blocos. Cada bloco é descrito por um hamiltoniano $H_{i}$ e a interação entre blocos vizinhos é feita por $V_{i j}=V_{i j} \delta_{i, j \pm 1}$.

eletrodos, podemos escrever a própria hamiltoniana da região de espalhamento em uma forma tridiagonal. Isso porque supomos que só há interação entre blocos vizinhos, o que de fato proporciona um ganho computacional ao resolver o problema. ${ }^{5}$ :

$$
H_{\mathrm{D}}=\left(\begin{array}{ccccccc}
H_{1} & V_{1,2} & 0 & 0 & 0 & \cdots & 0 \\
V_{2,1} & H_{2} & V_{2,3} & \cdots & 0 & \cdots & 0 \\
0 & V_{3,2} & \ddots & V_{j-1, j} & 0 & \cdots & 0 \\
\vdots & \vdots & V_{j, j-1} & H_{j} & V_{j, j+1} & \cdots & \vdots \\
0 & 0 & 0 & V_{j+1, j} & \ddots & \ddots & 0 \\
\vdots & \vdots & \vdots & \vdots & \ddots & H_{m-1} & V_{m-1, m} \\
0 & 0 & 0 & 0 & 0 & V_{m, m-1} & H_{m}
\end{array}\right) .
$$

\footnotetext{
${ }^{5}$ É importante notar que o hamiltoniano para a região central é finito, diferentemente do hamiltoniano apresentado na Eq. 3.18.
} 
Escrevendo a equação para a função de Green temos que:

$$
\left(\begin{array}{ccccccc}
\bar{H}_{1}-\Sigma_{\mathrm{L}} & \bar{V}_{1,2} & 0 & 0 & 0 & \cdots & 0 \\
\bar{V}_{2,1} & \bar{H}_{2} & \bar{V}_{2,3} & \ldots & 0 & \ldots & 0 \\
0 & \bar{V}_{3,2} & \ddots & \bar{V}_{j-1, j} & 0 & \ldots & 0 \\
\vdots & \vdots & \bar{V}_{j, j-1} & \bar{H}_{j} & \bar{V}_{j, j+1} & \cdots & \vdots \\
0 & 0 & 0 & \bar{V}_{j+1, j} & \ddots & \ddots & 0 \\
\vdots & \vdots & \vdots & \vdots & \ddots & \bar{H}_{m-1} & \bar{V}_{m-1, m} \\
0 & 0 & 0 & 0 & 0 & \bar{V}_{m, m-1} & \bar{H}_{m}-\Sigma_{\mathrm{R}}
\end{array}\right) \mathcal{G}=I,
$$

em que introduzimos as notações $\bar{H}_{i}=\left[E S_{i}-H_{i}\right]$ e $\bar{V}_{i, j}=\left[E S_{i, j}-V_{i, j}\right]$ para simplificar a escrita da matriz. Vale ressaltar que os blocos das extremidades $H_{1}$ e $H_{m}$ são os que estão acoplados aos eletrodos esquerdo e direito, respectivamente. Desta maneira, assim como fizemos na seção anterior, descrevemos os efeitos desses eletrodos na região de espalhamento (que agora é composta por $m$ blocos) pelas auto-energias, dadas pelas Eq. 3.34 e Eq. 3.35 .

A matriz que representa a função de Green (calculada pela Eq. 3.19) é dada por ${ }^{6}$ :

$$
\mathcal{G}=\left(\begin{array}{cccccccc}
\mathcal{G}_{1} & \mathcal{G}_{1,2} & \mathcal{G}_{1,3} & \ldots & \mathcal{G}_{1, j} & \ldots & \mathcal{G}_{1, m-1} & \mathcal{G}_{1, m} \\
\mathcal{G}_{2,1} & \mathcal{G}_{2} & \mathcal{G}_{2,3} & \ldots & \mathcal{G}_{2, j} & \ldots & \mathcal{G}_{2, m-1} & \mathcal{G}_{2, m} \\
\mathcal{G}_{3,1} & \mathcal{G}_{3,2} & \mathcal{G}_{3} & \ddots & \vdots & \mathcal{G}_{3, j+1} & \ldots & \mathcal{G}_{3, m} \\
\vdots & \vdots & \ddots & \ddots & \mathcal{G}_{j-1, j} & \mathcal{G}_{j-1, j+1} & \vdots & \vdots \\
\mathcal{G}_{j, 1} & \mathcal{G}_{j, 2} & \ldots & \mathcal{G}_{j, j-1} & \mathcal{G}_{j} & \mathcal{G}_{j, j+1} & \ldots & \mathcal{G}_{j, m} \\
\vdots & \vdots & \mathcal{G}_{j+1,3} & \mathcal{G}_{j+1, j-1} & \mathcal{G}_{j+1, j} & \ddots & \ddots & \vdots \\
\mathcal{G}_{m-1,1} & \mathcal{G}_{m-1,2} & \mathcal{G}_{m-1,3} & \ldots & \vdots & \ddots & \mathcal{G}_{m-1} & \mathcal{G}_{m-1, m} \\
\mathcal{G}_{m, 1} & \mathcal{G}_{m, 2} & \mathcal{G}_{m, 3} & \ldots & \mathcal{G}_{m, j} & \ldots & \mathcal{G}_{m, m-1} & \mathcal{G}_{m}
\end{array}\right)
$$

As matrizes de acoplamento $\Gamma$ entre os eletrodos e a região de espalhamento são dadas

\footnotetext{
${ }^{6}$ Apesar do hamiltoniano efetivo mostrado na Eq. 3.42 ser tridiagonal, a função de Green, em geral,
} não o será. 
por:

$$
\Gamma_{\mathrm{L}}=\left(\begin{array}{ccccccc}
i\left[\Sigma_{\mathrm{L}}-\Sigma_{\mathrm{L}}^{\dagger}\right] & 0 & 0 & 0 & 0 & \cdots & 0 \\
0 & 0 & 0 & \cdots & \cdots & \cdots & 0 \\
0 & 0 & \ddots & 0 & 0 & \cdots & 0 \\
\vdots & \vdots & 0 & 0 & 0 & \cdots & \vdots \\
0 & 0 & 0 & 0 & \ddots & \ddots & 0 \\
\vdots & \vdots & \vdots & \vdots & \ddots & 0 & 0 \\
0 & 0 & 0 & 0 & 0 & 0 & 0
\end{array}\right)
$$

e

$$
\Gamma_{\mathrm{R}}=\left(\begin{array}{ccccccc}
0 & 0 & 0 & 0 & 0 & \cdots & 0 \\
0 & 0 & 0 & \cdots & 0 & \cdots & 0 \\
0 & 0 & \ddots & 0 & 0 & \cdots & 0 \\
\vdots & \vdots & 0 & 0 & 0 & \cdots & \vdots \\
0 & 0 & 0 & 0 & \ddots & \ddots & 0 \\
\vdots & \vdots & \vdots & \vdots & \ddots & 0 & 0 \\
0 & 0 & 0 & 0 & 0 & 0 & i\left[\Sigma_{\mathrm{R}}-\Sigma_{\mathrm{R}}^{\dagger}\right]
\end{array}\right)
$$

Considerando os coeficientes de transmissão dados pela Eq. 3.37 e a estrutura das matrizes nas Eq. 3.43, Eq. 3.44 e Eq. 3.45, podemos chegar com um pouco de álgebra em:

$$
T(E)=\operatorname{Tr}\left[\Gamma_{\mathrm{L}} \mathcal{G}_{1 m} \Gamma_{\mathrm{R}} \mathcal{G}_{1 m}^{\dagger}\right]
$$

Isso significa que precisaríamos apenas dos elementos das extremidades $\left(\mathcal{G}_{1, m}\right)$ da matriz da Eq. 3.43, os quais são os termos da função de Green que conectam os dois eletrodos considerando o efeito da região de espalhamento.

O procedimento consiste em eliminar cada grau de liberdade (elementos da matriz da Eq. 3.43). Isso pode ser feito, considerando que cada bloco possa ser uma $C P$ (não são necessariamente iguais como na seção anterior). Assim, remove-se recursivamente cada grau de liberdade, renormalizando os acoplamentos e as energias de sítio até que se tenha apenas os elementos das extremidades desejados. Esse procedimento também é conhecido como decimação e é semelhante ao método de eliminação de Gauss. O processo de eliminação dos graus de liberdade se inicia pelo bloco com índice 2, que é o descrito 
pelo hamiltoniano $\bar{H}_{2}$,

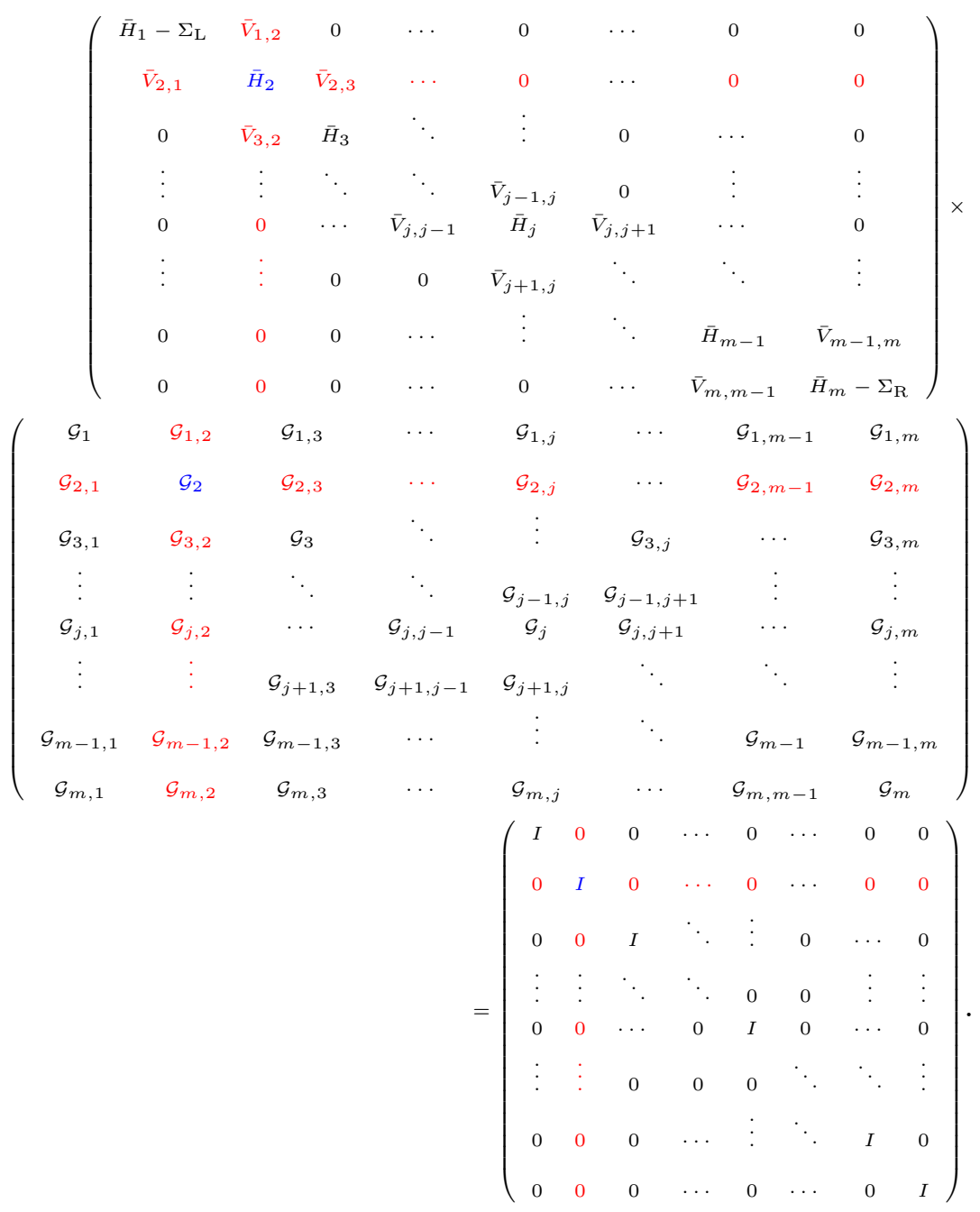

Após o primeiro processo de eliminação que denotamos com o superescrito 1 , temos os novos termos do hamiltoniano efetivo:

$$
\begin{aligned}
\bar{H}_{1}^{1} & =\bar{H}_{1}-\bar{V}_{1,2}\left[\bar{H}_{2}\right]^{-1} \bar{V}_{2,1}, \\
\bar{H}_{3}^{1} & =\bar{H}_{3}-\bar{V}_{3,2}\left[\bar{H}_{2}\right]^{-1} \bar{V}_{2,3} e \\
\bar{V}_{1,3}^{1} & =0-\bar{V}_{1,2}\left[\bar{H}_{2}\right]^{-1} \bar{V}_{2,3} ;
\end{aligned}
$$


desta forma obtemos uma nova forma para a Eq. 3.47 escrita por:

$$
\begin{aligned}
& \left(\begin{array}{ccccccc}
\bar{H}_{1}^{1}-\Sigma_{\mathrm{L}} & \bar{V}_{1,3}^{1} & \cdots & 0 & \cdots & 0 & 0 \\
\bar{V}_{3,1}^{1} & \bar{H}_{3}^{1} & \ddots & \vdots & 0 & \cdots & 0 \\
\vdots & \ddots & \ddots & \bar{V}_{j-1, j} & 0 & \vdots & \vdots \\
0 & \cdots & \bar{V}_{j, j-1} & \bar{H}_{j} & \bar{V}_{j, j+1} \cdots & 0 & \\
\vdots & 0 & 0 & \bar{V}_{j+1, j} & \ddots & \ddots & \vdots \\
0 & 0 & \cdots & \vdots & \ddots & \bar{H}_{m-1} & \bar{V}_{m-1, m} \\
0 & 0 & \cdots & 0 & \cdots & \bar{V}_{m, m-1} & \bar{H}_{m}-\Sigma_{\mathrm{R}}
\end{array}\right) \times
\end{aligned}
$$

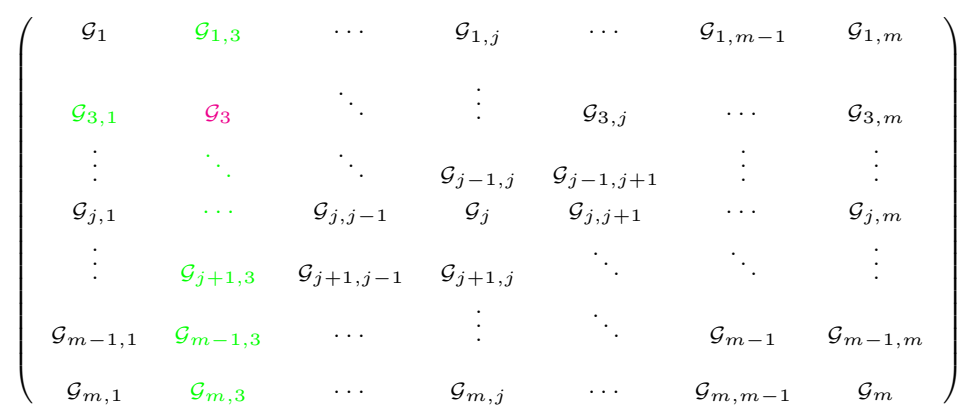

$$
\begin{aligned}
& =\left(\begin{array}{ccccccc}
I & 0 & \cdots & 0 & \cdots & 0 & 0 \\
& & \ddots & \vdots & 0 & \cdots & 0 \\
0 & I & \ddots & & & \vdots & \vdots \\
0 & \ddots & \ddots & 0 & 0 & \vdots & \vdots \\
0 & \cdots & 0 & I & 0 & \cdots & 0 \\
\vdots & 0 & 0 & 0 & \ddots & \ddots & \vdots \\
0 & 0 & \cdots & \vdots & \ddots & I & 0 \\
0 & 0 & \cdots & 0 & \cdots & 0 & I
\end{array}\right) .
\end{aligned}
$$

O procedimento é repetido $(m-2)$ vezes (lembrando que $m$ é o número de blocos da região de espalhamento), até que chegamos ao elemento $\bar{H}_{m-1}$. Após a eliminação de $(m-3)$ graus de liberdade, temos a seguinte forma para a Eq. 3.47:

$$
\left(\begin{array}{ccc}
\bar{H}_{1}^{(m-3)}-\Sigma_{\mathbf{L}} & \bar{V}_{1, m-1}^{(m-3)} & 0 \\
\bar{V}_{1, m-1}^{(m-3)} & \bar{H}_{m-1}^{(m-3)} & \bar{V}_{m-1, m} \\
0 & \bar{V}_{m, m-1} & \bar{H}_{m}-\Sigma_{\mathbf{R}}
\end{array}\right) \times\left(\begin{array}{ccc}
\mathcal{G}_{1} & \mathcal{G}_{1, m-1} & \mathcal{G}_{1, m} \\
\mathcal{G}_{m-1,1} & \mathcal{G}_{m-1} & \mathcal{G}_{m-1, m} \\
\mathcal{G}_{m, 1} & \mathcal{G}_{m, m-1} & \mathcal{G}_{m}
\end{array}\right)=\left(\begin{array}{ccc}
I & 0 & 0 \\
0 & I & 0 \\
0 & 0 & I
\end{array}\right) .
$$

A Fig. 3.7 mostra um resumo do esquema de decimação descrito acima. Depois do último processo de eliminação, temos:

$$
\left(\begin{array}{cc}
H_{1,1}^{\mathrm{eff}}-\Sigma_{\mathrm{L}} & H_{1, m}^{\mathrm{eff}} \\
H_{m, 1}^{\mathrm{eff}} & H_{m, m}^{\mathrm{eff}}-\Sigma_{\mathrm{R}}
\end{array}\right)\left(\begin{array}{cc}
\mathcal{G}_{1,1} & \mathcal{G}_{1, m} \\
\mathcal{G}_{m, 1} & \mathcal{G}_{m, m}
\end{array}\right)=\left(\begin{array}{cc}
I & 0 \\
0 & I
\end{array}\right) .
$$

Todo esse procedimento nos permite obter um hamiltoniano efetivo para a região de espalhamento (de um sistema contendo milhares de átomos) acoplada a dois eletrodos. 
a)

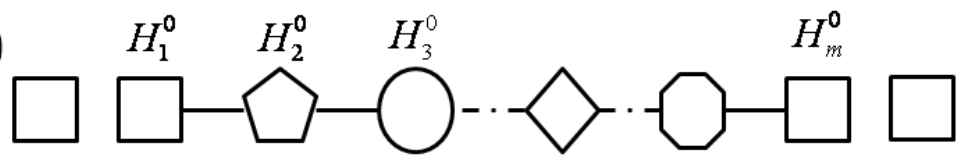

b)

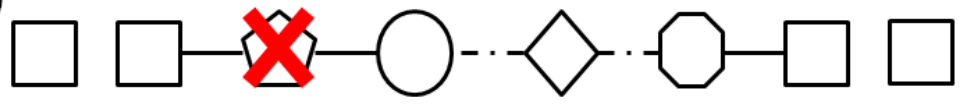

c)

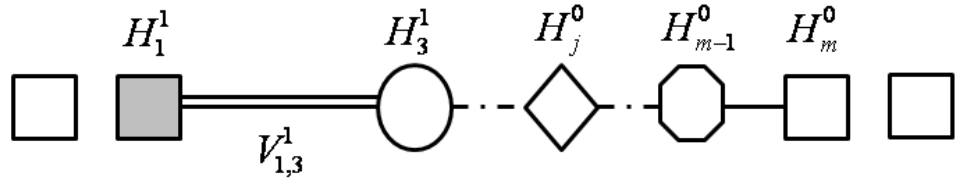

d) $\square \square$

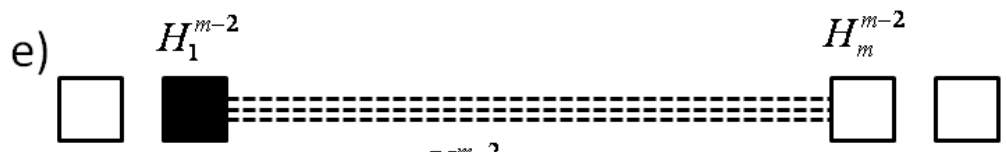

$V_{1, m-1}^{m-2}$

Figura 3.7: Esquema pictórico do processo de decimação: (a) m blocos da região de espalhamento; (b) o bloco descrito pelo hamiltoniano $\bar{H}_{2}$ é eliminado e o superescrito 1 é inserido para indicar a primeira etapa do processo; (c) a interação entre os blocos vizinhos é normalizada; (d) repete-se os passos (m-2) vezes até que (e) se chegue ao bloco que está acoplado ao eletrodo direito. Esta figura foi retirada da referência [34].

Os termos deste hamiltoniano efetivo são:

$$
\begin{aligned}
H_{1,1}^{\mathrm{eff}} & =\bar{H}_{1}^{m-2}=\bar{H}_{1}-\sum_{i=1}^{m-2} \bar{V}_{1, i+1}^{i-1}\left[\bar{H}_{i+1}^{i-1}\right]^{-1} \bar{V}_{i+1,1}^{i-1} \\
H_{m, m}^{\mathrm{eff}} & =\bar{H}_{m}^{m-2}=\bar{H}_{m}-\bar{V}_{m, m-1}^{0}\left[\bar{H}_{m-1}^{m-3}\right]^{-1} \bar{V}_{m-1, m}^{0} \\
H_{1, m}^{\mathrm{eff}} & =\bar{V}_{1, m}^{m-2}=-\bar{V}_{1, m-1}^{m-3}\left[H_{m-1}^{m-3}\right]^{-1} \bar{V}_{m-1, m}^{0}
\end{aligned}
$$

Podemos fazer mais uma simplificação ao considerarmos que os acoplamentos entre dois blocos quaisquer seja igual ao acoplamento entre dois blocos de nanotubos puros, ou seja, igual a interação entre duas $C P$ no caso de um único defeito na região de espalhamento. Isso pode ser feito pelo seguinte motivo: cada hamiltoniano $\bar{H}_{i}$ de cada bloco é obtido por um cálculo de DFT separadamente. Quando fazemos esse cálculo para um bloco contendo um defeito, esse bloco contém a parte central do defeito onde a 
molécula irá se dissociar mais uma parte de nanotubo puro de cada lado. Isso é feito para que não exista interação entre o defeito e sua imagem devido às condições periódicas de contorno. Consequentemente, cada bloco de defeito tem as extremidades idênticas a um bloco de nanotubo puro, de forma que podemos considerar que os acoplamentos entre eles sejam realmente iguais aos acoplamentos entre blocos estritamente de nanotubos puros. Portanto, a densidade de defeitos não pode ser alta, como será o caso neste trabalho.

Sendo assim, o cálculo da função de Green do sistema contendo milhares de átomos pode ser reduzido, após o procedimento de decimação, à solução de uma equação idêntica à Eq. 3.32 com as auto-energias dadas pelas Eq. 3.34 e Eq. 3.35. Finalmente, os coeficientes de transmissão podem ser calculados pela Eq. 3.37 e a condutância, no limite de $V \rightarrow 0$, pela Eq. 3.15.

Na realidade, a grandeza que estamos interessados em calcular é a condutância diferencial $g=d I / d V$. No regime linear, podemos utilizar as equações (3.37) e (3.38) para obtermos $g$ :

$$
g=\lim _{V \rightarrow 0} \frac{\partial I}{\partial V}=-\left.\frac{e^{2}}{h} \int T(E) \frac{\partial f\left(E^{\prime}, \mu_{0}\right)}{\partial E^{\prime}}\right|_{E^{\prime}=E} d E .
$$

Se aplicarmos a derivada, podemos escrever a condutância diferencial por:

$$
g=\frac{e^{2}}{4 k_{B} \Theta h} \int T(E) \operatorname{sech}^{2}\left(\frac{E-\mu_{0}}{2 k_{B} \Theta}\right) d E .
$$

Em geral, a transmitância pode apresentar variações drásticas em função da energia, principalmente para sistemas com um número muito grande de defeitos na região de espalhamento. Desta forma, para efetuarmos a integração da Eq. 3.58, seria necessário um número muito grande de pontos. Entretanto, se usarmos o fato de que

$$
\frac{d \tanh (x)}{d(x)}=\operatorname{sech}^{2}(x)
$$

podemos fazer uma mudança de variável $y=\tanh \left(\frac{E-\mu_{0}}{2 k_{B} \Theta}\right)$ e escrever:

$$
g=\frac{e^{2}}{2 h} \int_{-1}^{1} T\left(2 k_{B} \Theta \operatorname{arctanh}(y)+\mu_{0}\right) d y .
$$

Além da mudança dos limitites de integração que sai de um intervalo aberto $(-\infty,+\infty)$ para um intervalo fechado $[-1,1]$, a principal vantagem que essa mudança de variável trás é que quando tomamos pontos igualmente espaçados no eixo vertical, a maior concentração de pontos se encontra para energias em torno do nível de Fermi, conforme mostrado na Fig. 3.8. Desta maneira, se utilizássemos a variável $E$ como variável de integração, 


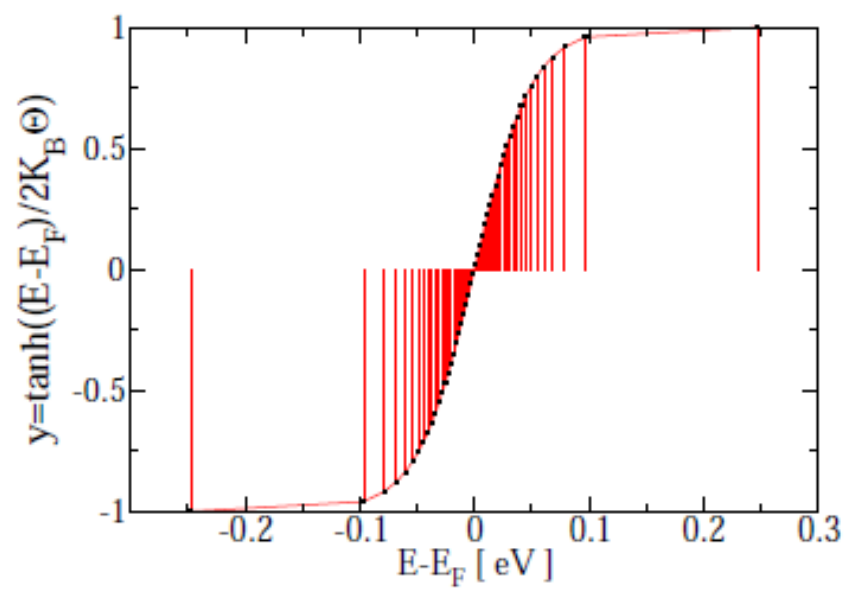

Figura 3.8: Plote da função transformação $y$ em função da energia para $k_{B} \Theta=50 \mathrm{meV}$. Os pontos de integração estão igualmente espaçados. A maior concentração de pontos fica em torno do nível de Fermi. Esta figura foi retirada da referência [34].

teríamos que tomar muito mais pontos para descrever com a mesma precisão quando efetuamos a integração na variável $y$. Como a quantidade de pontos de integração está intimamente ligada com o custo computacional, com essa mudança de variável podemos calcular sistemas muito maiores. 


\section{Capítulo 4}

\section{Propriedades Estruturais e}

\section{Energéticas}

\subsection{Estudo do processo de dissociação de moléculas na região do defeito $N R$}

Antes de iniciarmos os cálculos de relaxação dos sistemas (nanotubo com defeito + molécula), cada uma das moléculas mostradas na Fig. 1.6 teve a geometria otimizada utilizando os parâmetros listados no apêndice B. Algumas propriedades como comprimento de ligação $\left(d_{a-b}\right)$ entre os átomos $a$ e $b$, e ângulos $\operatorname{diedros}(\theta)$ são apresentadas na Tab. 4.1. Em seguida, as estruturas otimizadas das moléculas isoladas foram usadas para

Tabela 4.1: Propriedades estruturais das moléculas isoladas: comprimento de ligação $\left(d_{a-b}\right)$, ângulos diedros $(\theta)$.

\begin{tabular}{ccr}
\hline \hline Molécula & $d_{a-b}(\AA)$ & $\theta$ \\
\hline $\mathrm{H}_{2} \mathrm{O}$ & 0.98 & $104.2^{\circ}$ \\
$\mathrm{N}_{2}$ & 1.11 & - \\
$\mathrm{O}_{2}$ & 1.24 & - \\
$\mathrm{H}_{2}$ & 0.79 & - \\
$\mathrm{H}_{2} \mathrm{~S}$ & 1.37 & $91.2^{\circ}$ \\
$\mathrm{CO}$ & 1.19 & $180^{\circ}$ \\
$\mathrm{CO}$ & 1.16 & - \\
$\mathrm{NH}_{3}$ & 1.03 & $105.7^{\circ}$ \\
\hline \hline
\end{tabular}

montar as geometrias dos sistemas (nanotubo com defeito + molécula).

Partindo da estrutura modelo da Fig. 4.2, estudamos a possibilidade das moléculas 


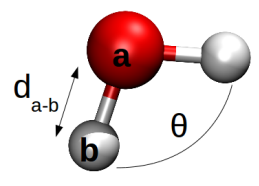

Figura 4.1: Ilustração das grandezas comprimento de ligação $d_{a-b}$ entre os átomos $a$ e $b$ e ângulo diêdro $\theta$ para a molécula de água.

mostradas na Fig. 1.6 se ligarem na região de defeito do nanotubo $(N R)$. O procedimento ideal para se estudar o processo de dissociação das moléculas na superfície do nanotubo seria determinar a superfície de potencial de cada um dos sistemas $N R+$ molécula, o que possibilitaria identificar onde e como a molécula se ligaria. Entretanto, é um procedimento complicado e computacionalmente muito caro. O critério obedecido para montagem das estruturas foi posicionar a molécula próxima ao defeito de maneira que a(s) distância(s) do(s) átomo(s) da molécula mais próximo(s) aos átomos do defeito fosse(m) aproximadamente $10 \%$ a $15 \%$ maiores que as distâncias de ligação entre os átomos da molécula. Para

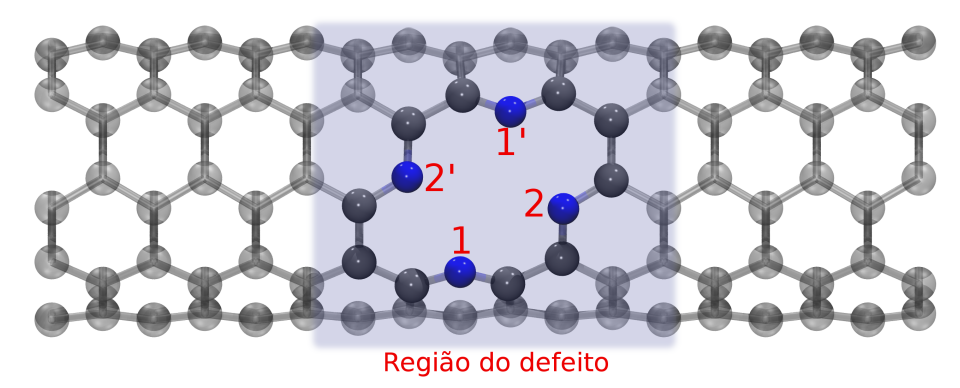

Figura 4.2: Sistema $N R$ para o nanotubo $(5,5)$ contendo 178 átomos. O defeito é formado pela divacância de carbono e 4 átomos de nitrogênio substitucionais. Os rótulos nos átomos de nitrogênio indicam os sítios equivalentes: $1 \equiv 1^{\prime}$ e $2 \equiv 2^{\prime}$.

cada sistema $N R+$ molécula foram montadas de 3 a 5 destas possíveis configurações e em seguida foi feita a relaxação pelo método de gradiente conjugado de cada sistema a fim de se obter a estrutura de mais baixa energia. Foram investigadas as propriedades estruturais e energéticas para essas configurações.

Com o intuito de diminuir o custo computacional do processo de relaxação, foram feitos testes em que relaxamos todos os átomos e comparamos com o caso em que fixamos 3 células unitárias de cada lado nas extremidades do $N R$ permitindo a relaxação apenas 
da região central. Observamos que a perturbação causada pela molécula fica concentrada na região do defeito de maneira que os átomos de carbono mais distantes praticamente não se movimentam. Desta forma, fizemos todas as relaxações seguindo esse procedimento.

\subsubsection{Primeiro grupo de moléculas: Principais constituintes do ar atmosférico}

O ar atmosférico, em condições normais de temperatura e pressão (CNTP), é constituído basicamente por $78.08 \%$ de nitrogênio, $20.94 \%$ de oxigênio, $0.93 \%$ de argônio, $0.03 \%$ de dióxido de carbono, vapor de água em torno de $1 \%$ próximo à superfície e porcentagens muito menores de outros gases nobres. Conforme discutido no final da seção 1.2.1, a motivação para a escolha das moléculas $\mathrm{H}_{2} \mathrm{O}, \mathrm{O}_{2}$ e $\mathrm{N}_{2}$ baseia-se no fato de que para uma possível aplicação do sistema $N R$ como sensor de gás seria importante que os principais constituintes do ar atmosférico não interferissem nas medidas, ou seja, que estas moléculas não fossem detectadas.

\section{Água $\left(\mathrm{H}_{2} \mathrm{O}\right)$}

Para a molécula de $\mathrm{H}_{2} \mathrm{O}$ fizemos a relaxação de 5 estruturas conforme mostra a Fig. 4.3. Nos quatro casos apresentados na Fig. 4.3(b-e) não observamos dissociação da molécula na região de defeito. Desta maneira, foi feito um cálculo de relaxação em que a molécula estava inicialmente dissociada, conforme a estrutura mostrada na Fig. 4.3(a). A Tab. 4.2 mostra as energias de ligação ${ }^{1}$ das estruturas estudadas nesse trabalho. Os resultados mostram que todas os sistemas tem energia de ligação variando de $-0.45 \mathrm{eV}$ a $-0.26 \mathrm{eV}$. Nossos resultados indicam que o processo de dissociação da molécula pode apresentar barreira de potencial. Para as estruturas mostradas nas Figs. 4.3(b-e), vemos que as energias de ligação são muito próximas. Isso indica que a superfície de potencial possui mínimos rasos. Em particular as estruturas relaxadas das figuras 4.3(c) e 4.3(d)

\footnotetext{
${ }^{1}$ O SIESTA usa conjunto de bases numéricas e localizadas nos átomos, o que faz aparecer uma complicação para calcular as energias de ligação uma vez que o conjunto de bases não é completo. Desta forma, faz-se necessária a correção do "erro de superposição de base" (BSSE)[62] que é feita da seguinte maneira: seja um sistema $A B$ constituído de dois sub-sistemas $A$ e $B$; seja $E^{X}(Z)$ a energia total do sub-sistema $Z$ relaxada com a base $X$. A energia de ligação desse sistema considerando a correção BSSE é $E_{b}(A B)=$ $\left[E^{A B}(A B)-E^{A}(A)-E^{B}(B)\right]+\delta_{A B}^{B S S E}$, em que $\delta_{A B}^{B S S E}=\left[\left(E^{A}(A)-E^{A B}(A)\right)+\left(E^{B}(B)-E^{A B}(B)\right)\right]$.
} Em outras palavras, todas as partes devem ser calculadas com o mesmo tamanho de base. 


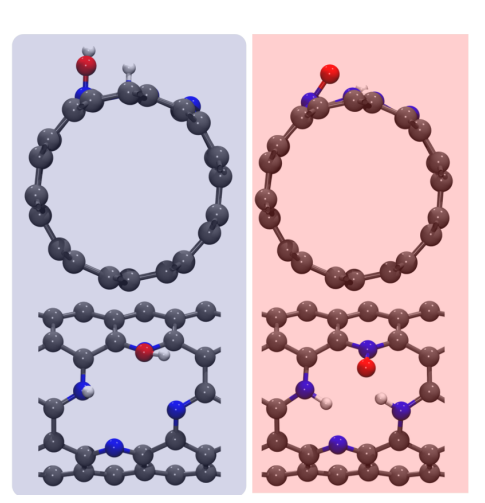

(a)

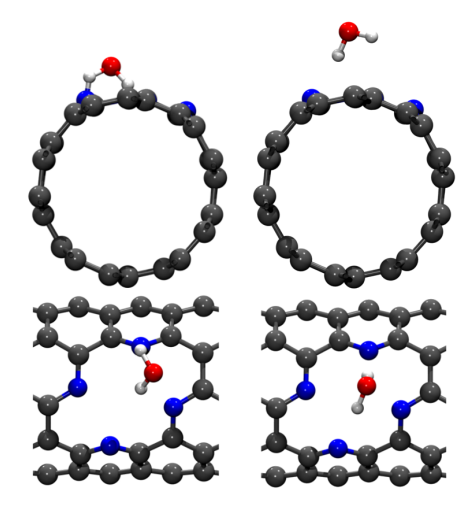

(b)

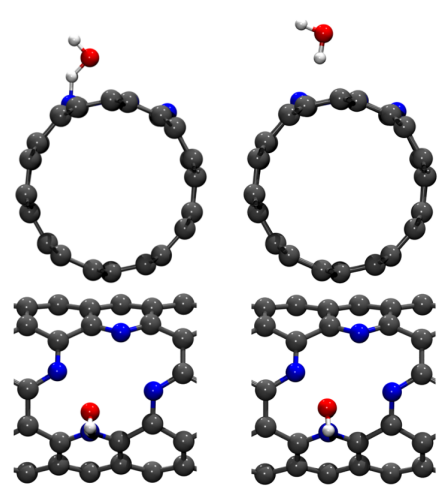

(c)

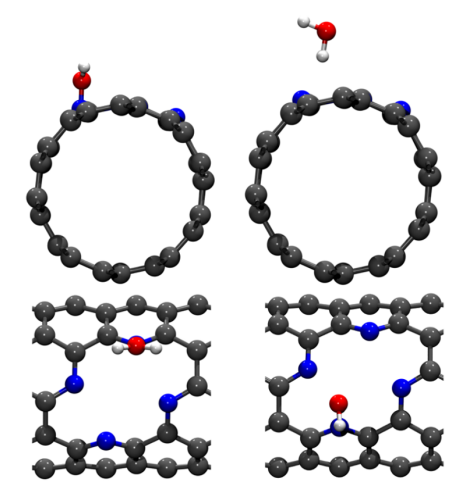

(d)

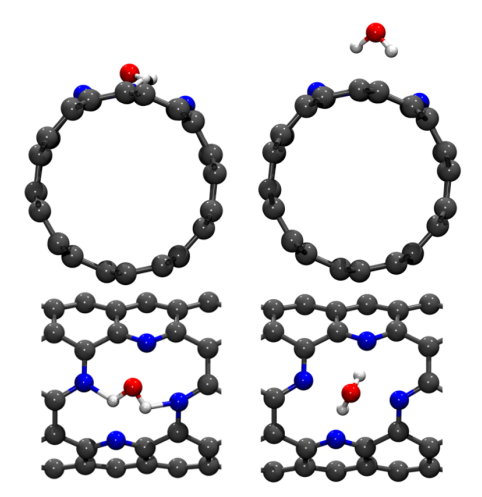

(e)

Figura 4.3: Estruturas iniciais (painel esquerdo - coluna azul) e relaxadas (painel direito - coluna vermelha) para os 5 sistemas testados $\mathrm{NR}+\mathrm{H}_{2} \mathrm{O}$. Em (a) a molécula foi posicionada inicialmente dissociada. Painéis superiores e inferiores de cada coluna correspondem à mesma estrutura sob diferentes pontos de vista.

são muito parecidas, e que possivelmente seriam exatamente iguais se os critérios de convergência de forças fossem mais refinados. As energias de ligação são calculadas pela Eq.

Tabela 4.2: Energia de ligação/dissociação $\left(E_{b}\right)$ em unidades de $e V$ das estruturas relaxadas.

\begin{tabular}{cccccc}
\hline \hline Molécula & \multicolumn{5}{c}{ Estruturas } \\
& $(a)$ & $(b)$ & $(c)$ & $(d)$ & $(e)$ \\
\hline $\mathrm{H}_{2} \mathrm{O}$ & -0.45 & -0.33 & -0.26 & -0.26 & -0.34 \\
$\mathrm{~N}_{2}$ & 2.95 & 2.95 & $\simeq 0$ & $\simeq 0$ & - \\
$\mathrm{O}_{2}$ & $\simeq 0$ & -0.68 & $\simeq 0$ & $\simeq 0$ & - \\
$\mathrm{H}_{2}$ & -2.66 & $\simeq 0$ & $\simeq 0$ & $\simeq 0$ & - \\
$\mathrm{H}_{2} \mathrm{~S}$ & -1.11 & -0.19 & -0.17 & -0.19 & - \\
$\mathrm{CO}_{2}$ & 3.59 & $\simeq 0$ & 0.35 & - & - \\
$\mathrm{CO}$ & -0.41 & -0.41 & -0.41 & - & - \\
$\mathrm{NH}_{3}$ & -0.59 & -0.26 & -0.16 & -0.18 & -0.17 \\
\hline \hline
\end{tabular}


4.1, e é definida como sendo a diferença de energia entre a energia total do sistema composto $(N R+$ molécula $)$ pela soma das energias totais dos sub-sistemas ( $N R$ e molécula) isolados.

$$
E_{b}=E_{\text {total }}(\mathrm{NR}+\text { molécula })-E_{\text {total }}(N R)-E_{\text {total }}(\text { molécula }) .
$$

É interessante observar que as energias de ligação resultantes para as estruturas da água são altas quando comparadas às energias de ligação de sistemas fisisorvidos que são da ordem de $k_{B} \Theta \sim 0.025 \mathrm{eV}$. No caso da primeira estrutura, esta constatação pode ser explicada pelo simples fato da molécula estar dissociada. Por outro lado, as quatro últimas estruturas estão interagindo por meio de ligações de hidrogênio, em que o(s) hidrogênio(s) da molécula estão direcionados para o(s) átomo(s) de nitrogênio da região de defeito. A distância entre os átomos de hidrogênio da molécula e os átomos de nitrogênio do nanotubo variam entre $1.95 \AA$ a $2.3 \AA$. O comprimento das ligações de hidrogênio varia de sistema para sistema e com as condições que cada sistema está submetido. Para citar alguns exemplos de comprimentos de ligação de hidrogênio em diferentes sistemas, ver as referências $[63,64,65]$.

\section{Nitrogênio $\left(N_{2}\right)$}

Para a molécula de $N_{2}$ fizemos a relaxação de 4 estruturas conforme a Fig. 4.4. Em particular, as estruturas das Figs. 4.4(a-b) apresentaram a mesma configuração após a relaxação. Entretanto, a energia de ligação calculada para essas configurações foi de $2.95 \mathrm{eV}$. Esses resultados permitem-nos concluir que, apesar dessa configuração ser um mínimo local, a molécula de $N_{2}$ não se liga à região do defeito, pois a energia que o sistema ganha para qua a molécula fique nessa configuração é muito alta. Esse resultado é desejável para uma possível aplicação deste sistema como sensor de gás, já que o nitrogênio é o principal constituinte do ar atmosférico.

Para as duas últimas estruturas mostradas nas Figs. 4.4(c-d), a molécula de $N_{2}$ se afasta do nanotubo e as energias de ligação associadas à essas estruturas são aproximadamente zero. Elas não são exatamente iguais e isso pode estar associado aos critérios de convergências escolhidos para as forças. 


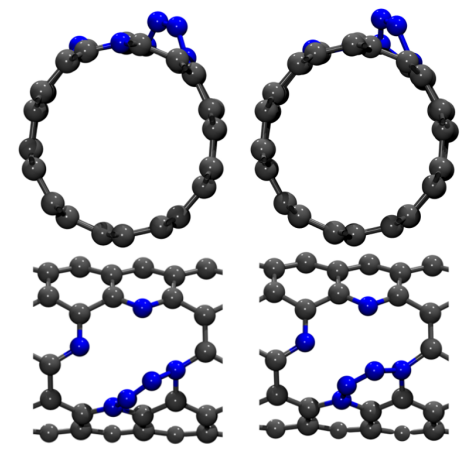

(a)

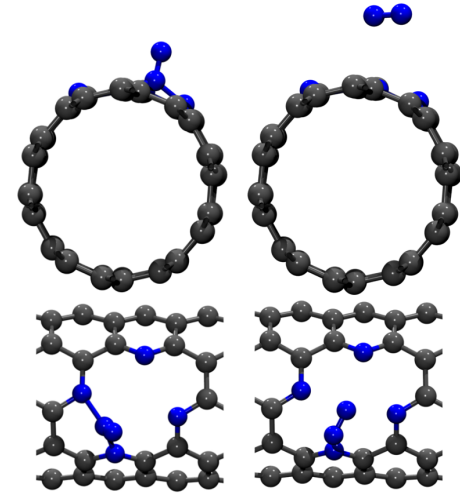

(c)

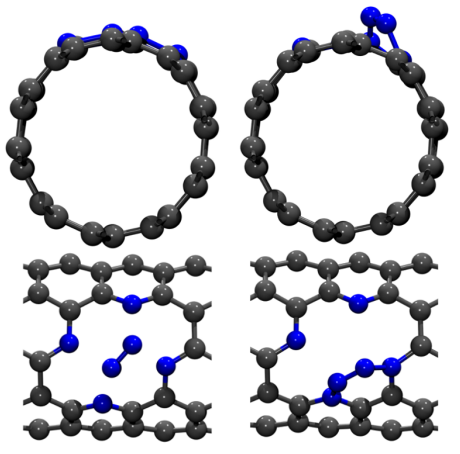

(b)

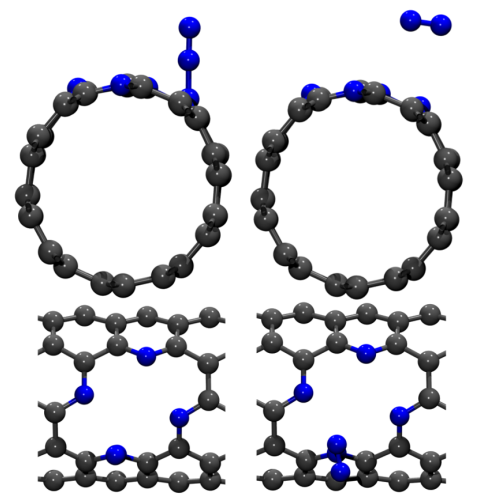

(d)

Figura 4.4: Estruturas iniciais (painel esquerdo) e relaxadas (painel direito) para os 4 sistemas testados $N R+N_{2}$. Painéis superiores e inferiores de cada coluna correspondem à mesma estrutura sob diferentes pontos de vista.

\section{Oxigênio molecular $\left(\mathrm{O}_{2}\right)$}

As principais estruturas estudadas para a molécula de $O_{2}$ estão mostradas na Fig. 4.5; duas delas (Fig. 4.5(a) e Fig. 4.5(b)) dissociaram e as energias envolvidas são $\simeq 0$ e $-0.68 \mathrm{eV}$, respectivamente. Para o primeiro caso, a energia associada ao processo de dissociação é menor do que a energia térmica à temperatura ambiente, consequentemente, essa configuração provavelmente não seria observada. Em particular para a última, também foram feitos cálculos de relaxação de outras possibilidades em que variamos a altura da molécula em relação ao plano do defeito. Em todos esses casos em que a molécula foi posicionada acima do plano ela se afastou do nanotubo, o que indica que possivelmente existe uma barreira de potencial a ser vencida para que a dissociação ocorra. Para as demais estruturas mostradas nas Figs. 4.5(c-d), vemos que a molécula se afastou do nanotubo e 


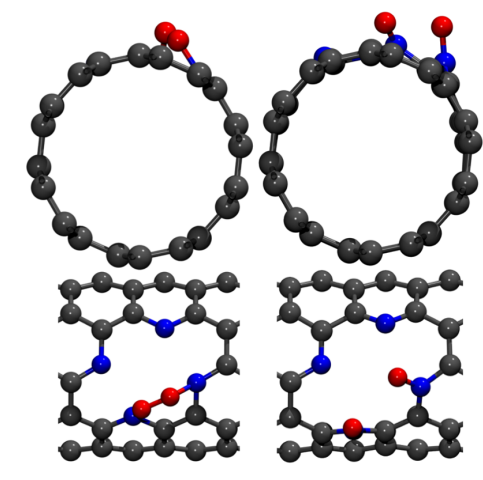

(a)

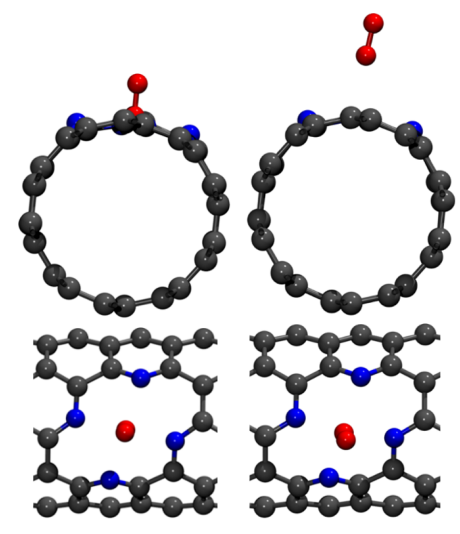

(c)

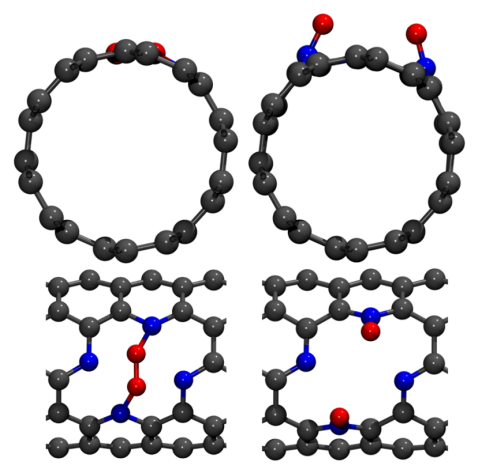

(b)

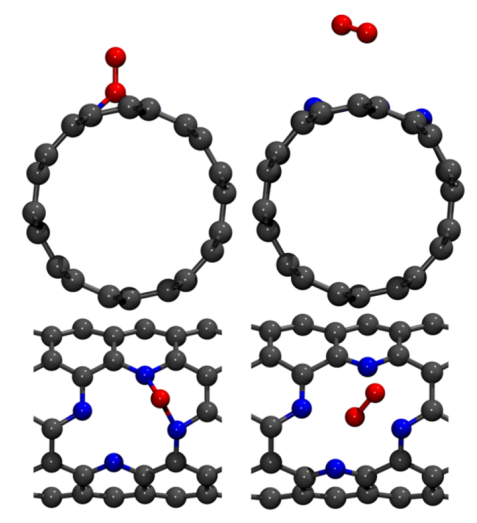

(d)

Figura 4.5: Estruturas iniciais (painel esquerdo) e relaxadas (painel direito) para 4 sistemas $\mathrm{NR}+\mathrm{O}_{2}$. Painéis superiores e inferiores de cada coluna correspondem à mesma estrutura sob diferentes pontos de vista.

o fato de as configurações relaxadas serem diferentes pode, novamente, estar relacionado com os critérios de convergências para as forças.

O $\mathrm{O}_{2}$ é o único entre todos os gases investigados neste trabalho que apresenta estado tripleto como estado fundamental. Entretanto, quando a molécula se dissocia, o sistema final não apresenta magnetização total ou local $(\operatorname{spin}[\uparrow]-\operatorname{spin}[\downarrow]=0)$ e isso sugere que a molécula deve mudar de estado tripleto para singleto durante o processo de aproximação. Seria interessante, como perspectivas futuras, estudarmos as barreiras de potencial em função da distância de aproximação da molécula à região de defeito, tanto para o caso singleto como para o tripleto. Nesse caso, a barreira de potencial deve incluir a interação spin-órbita a fim de capturar corretamente esta transição tripleto-singleto. 
Hidrogênio $\left(H_{2}\right)$

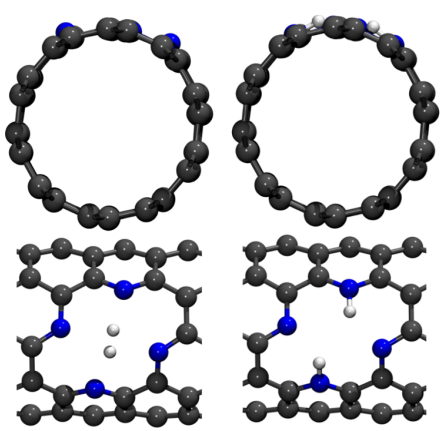

(a)

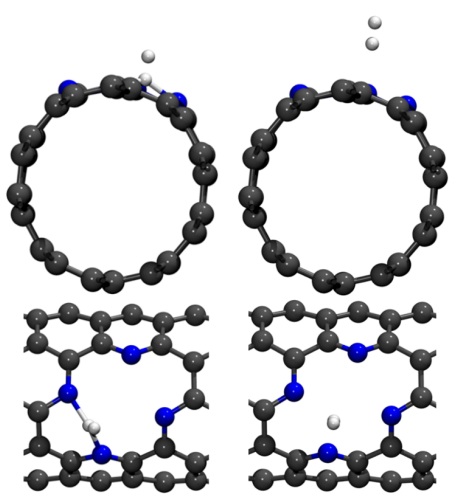

(c)

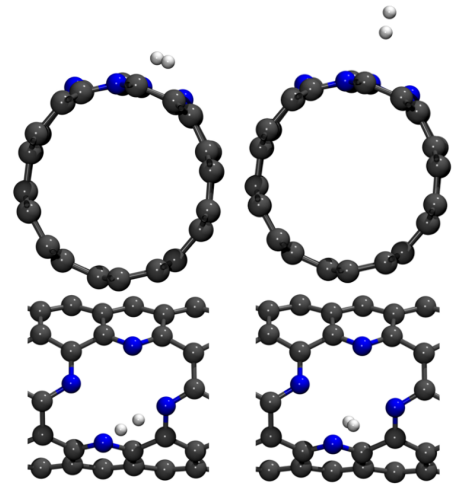

(b)

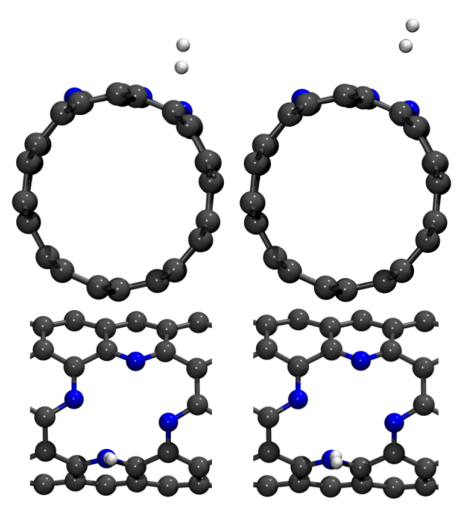

(d)

Figura 4.6: Estruturas iniciais (painel esquerdo) e relaxadas (painel direito) para os 4 sistemas testados $\mathrm{NR}+\mathrm{H}_{2}$. Painéis superiores e inferiores de cada coluna correspondem à mesma estrutura sob diferentes pontos de vista.

Para a molécula de $H_{2}$ fizemos a relaxação de 4 estruturas conforme mostrado na Fig. 4.6 e apenas a estrutura mostrada na Fig. 4.6(a) dissociou. É importante observar que a energia do sistema abaixa de $-2.66 \mathrm{eV}$ após a dissociação da molécula quando comparada com a soma dos sub-sistemas calculados isoladamente. No entanto, ao posicionarmos a molécula de $H_{2}$ arbitrariamente acima do plano do defeito, observamos em todos os casos, que a molécula se afasta do nanotubo.

Novamente o que podemos concluir é que possivelmente existe uma barreira para a dissociação da molécula $\mathrm{H}_{2}$ e que precisaria ser determinada para inferir qual a probabilidade dessa configuração dissociada ocorrer. As demais estruturas relaxadas mostradas nas Figs. 4.7(b-d) são muito parecidas e, novamente, as pequenas diferenças podem estar 
relacionadas com o critério de convergência escolhido para as forças.

\subsubsection{Segundo grupo de moléculas: Alguns gases poluentes}

\section{Ácido sulfídrico $\left(\mathrm{H}_{2} \mathrm{~S}\right)$}

Para a molécula de $\mathrm{H}_{2} S$ foram estudadas 4 estruturas, as quais foram montadas de maneira análoga às estruturas inicias da água, conforme observamos na Fig. 4.7. As estruturas apresentadas nas Fig. 4.7(b-d) foram relaxadas e, como aconteceu para a molécula de água, em nenhuma delas a molécula se dissociou na região de defeito. As

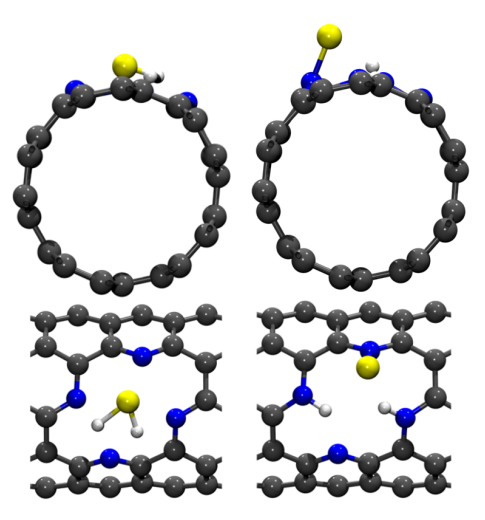

(a)

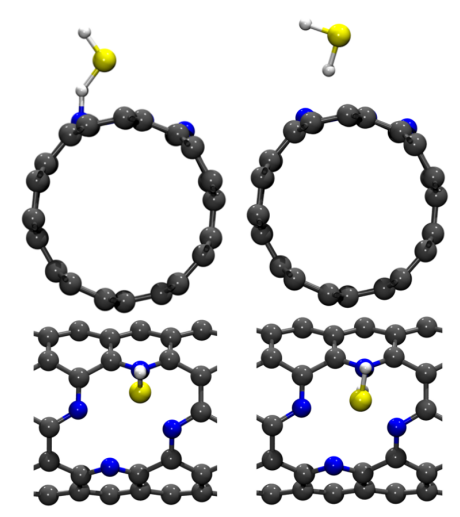

(c)

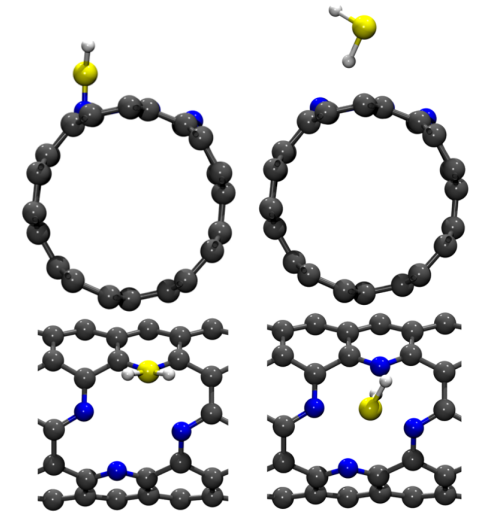

(b)

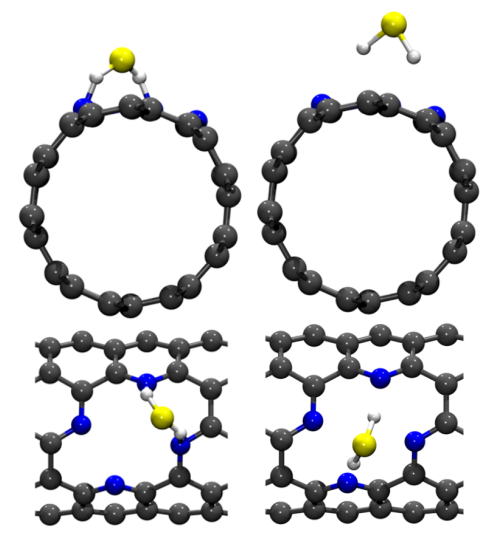

(d)

Figura 4.7: Estruturas iniciais (painel esquerdo) e relaxadas (painel direito) para os 4 sistemas $\mathrm{NR}+\mathrm{H}_{2} \mathrm{~S}$. Painéis superiores e inferiores de cada coluna correspondem à mesma estrutura sob diferentes pontos de vista.

energias de ligação calculadas para essas estruturas foram de $\sim-0.19 \mathrm{eV}$. Como ocorreu para a molécula de água, há a formação de ligações de hidrogênio entre os átomos de 
hidrogênio da molécula e os átomos de nitrogênio da região de defeito do nanotubo que mantém a molécula a uma distância típica de aproximadamente $2.3 \AA$. Em particular, as estruturas relaxadas mostradas nas Figs. 4.7(b-c) são muito parecidas, o que indica uma superfície de potencial com mínimos rasos. Se os critérios de convergências das forças fossem mais refinados, possivelmente estas estruturas seriam idênticas.

Para a estrutura mostrada na Fig. 4.7(a), iniciamos a relaxação com a molécula já dissociada e observamos que um segundo hidrogênio da molécula é também removido. A estrutura final obtida é semelhante à Fig. 4.3(a) para a molécula de água. A energia de ligação associada a esse processo é de $-1.19 \mathrm{eV}$.

\section{Dióxido de carbono $\left(\mathrm{CO}_{2}\right)$}

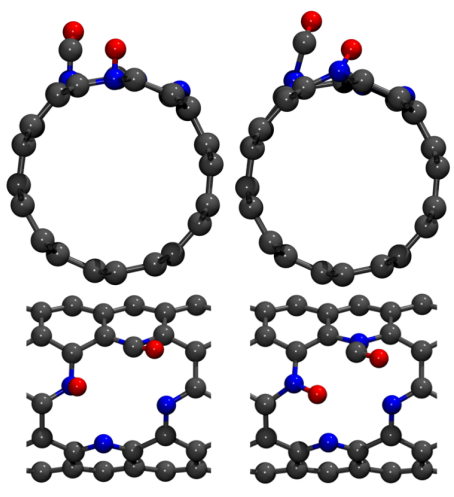

(a)

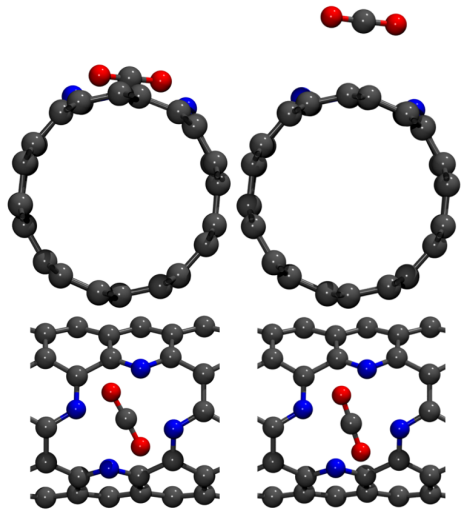

(b)

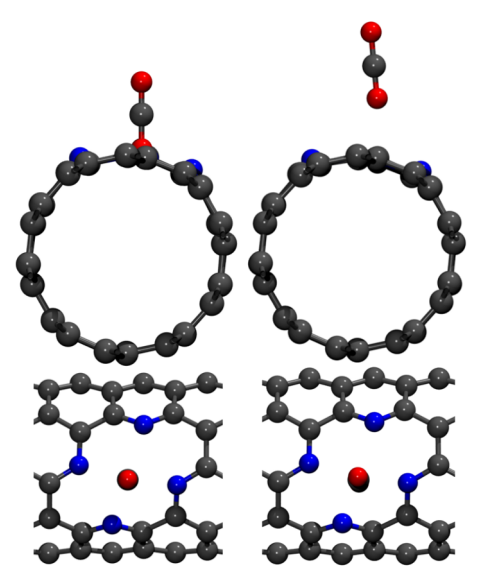

(c)

Figura 4.8: Estruturas iniciais (painel esquerdo) e relaxadas (painel direito) para os sistemas $\mathrm{NR}+\mathrm{CO}_{2}$. Painéis superiores e inferiores de cada coluna correspondem à mesma estrutura sob diferentes pontos de vista.

Por ser uma molécula grande (quando comparada com as apresentadas anteriormente) e linear, foram feitos cálculos de relaxação de apenas 3 estruturas, as quais estão mostradas na Fig. 4.8; em nenhum dos casos a molécula se ligou/dissociou na região de defeito do nanotubo. Seguindo o procedimento adotado para as anteriores que também não apresentaram dissociação, foi feita a relaxação de uma estrutura já inicialmente dissociada, Fig. 4.8(a). A energia de ligação para essa estrutura foi de $3.59 \mathrm{eV}$, conforme mostrado na Tab. 4.2. Isso permite-nos concluir que a molécula de $\mathrm{CO}_{2}$ não se dissociaria na região de defeito do nanotubo e consequentemente esse gás não seria detectado pelo 
sistema.

\section{Monóxido de carbono $(C O)$}

Para a molécula de $C O$ fizemos cálculos de relaxação de várias estruturas (Fig. 4.9) e esta foi a única que se ligou à região do defeito do nanotubo em todos os casos testados. Mesmo quando iniciamos os cálculos com a molécula dissociada (Fig. 4.9(a)), os átomos se recombinaram e a estrutura final foi a mesma em todos os casos, com o átomo de carbono da molécula ligado a dois nitrogênios da região do defeito. Para esse sistema, fizemos o

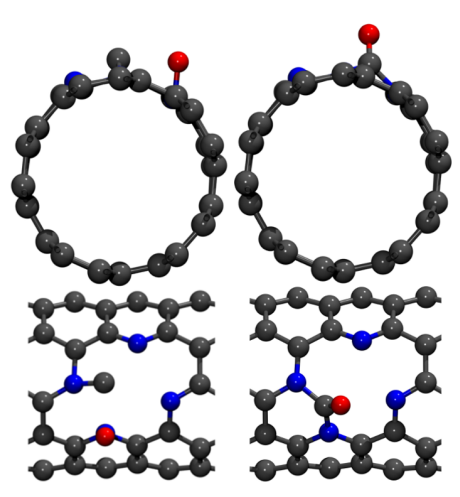

(a)

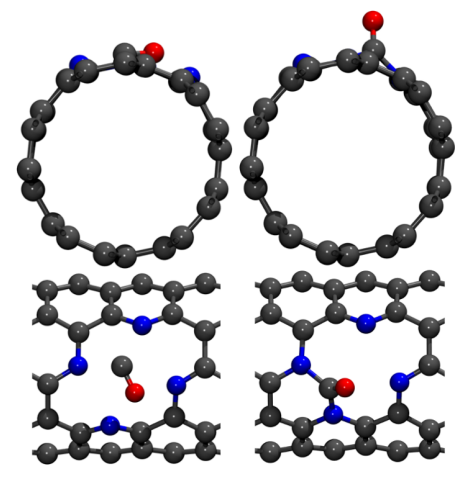

(b)

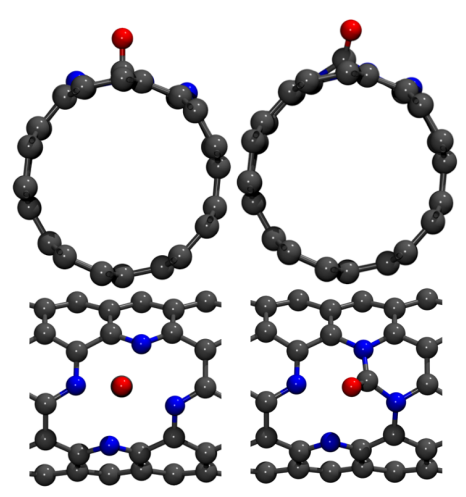

(c)

Figura 4.9: Estruturas iniciais (painel esquerdo) e relaxadas (painel direito) para os sistemas $N R+C O$. Painéis superiores e inferiores de cada coluna correspondem à mesma estrutura sob diferentes pontos de vista.

cálculo da energia de ligação e o resultado foi de $E_{b}=-0.41 \mathrm{eV}$. Estes resultados destacam o $C O$ dos demais gases estudados até agora pois ele se liga ao defeito sem barreira de potencial, o que em princípio seria interessante para a detecção deste gás pelo nanotubo. Nota-se que para a estrutura mostrada na Fig. 4.9(c), a molécula de $C O$ se liga em um sítio equivalente àquele da Fig. 4.9(a), de forma que a energia de ligação é a mesma em todos os casos.

\section{Amônia $\left(\mathrm{NH}_{3}\right)$}

Para a molécula de $\mathrm{NH}_{3}$ fizemos cálculos de relaxação das 5 estruturas mostradas na Fig. 4.10. Para as duas estruturas apresentadas nas Fig. 4.10(a) e Fig. 4.10(b), a molécula se dissociou e as energias envolvidas nesses processos foram $-0.59 \mathrm{eV}$ e $-0.26 \mathrm{eV}$, 
respectivamente. Em particular, a primeira estrutura foi a mesma encontrada nos cálculos

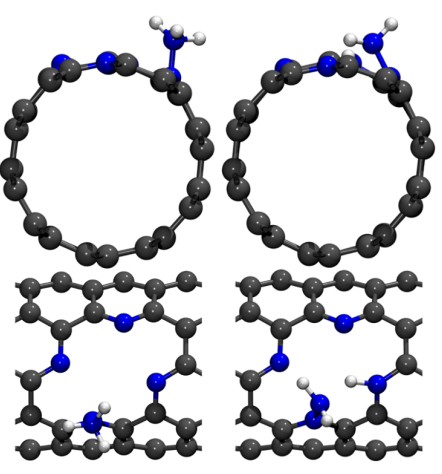

(a)

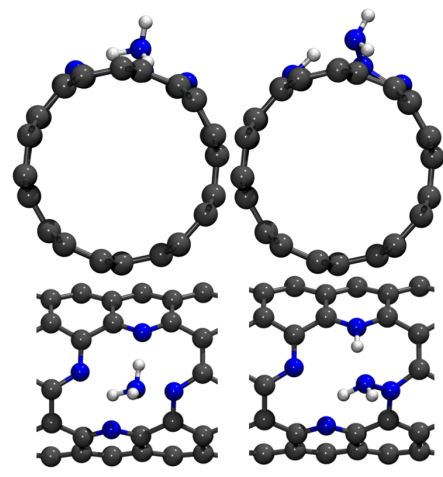

(b)

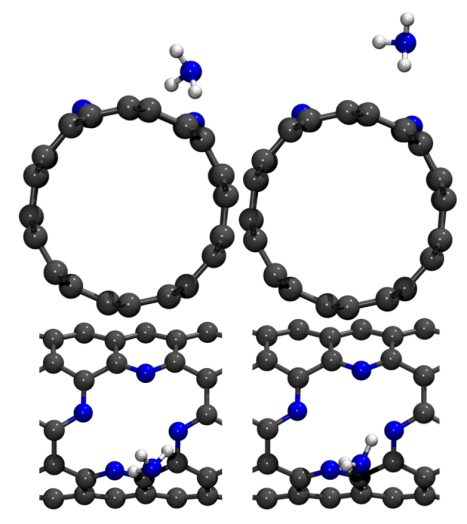

(c)

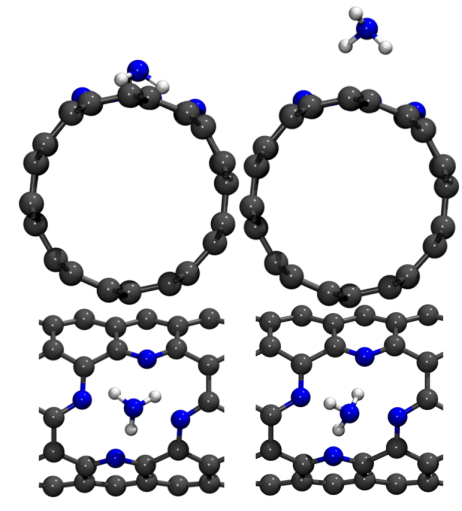

(d)

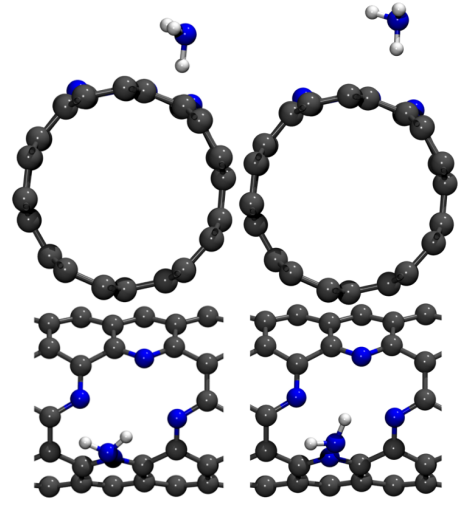

(e)

Figura 4.10: Estruturas iniciais (painel esquerdo) e relaxadas (painel direito) para os sistemas testados $\mathrm{NR}+\mathrm{NH}_{3}$. Painéis superiores e inferiores de cada coluna correspondem à mesma estrutura sob diferentes pontos de vista.

anteriores realizados no nosso grupo [35] utilizando ondas planas ${ }^{2}$. Para as demais estruturas apresentadas nas Fig. 4.10(c-e), os cálculos de energia de ligação mostraram valores que variam entre $-0.18 \mathrm{eV}$ a $-0.16 \mathrm{eV}$. Isso pode ser atribuído à formação de ligações de hidrogênio entre os átomos de hidrogênio da molécula e os átomos de nitrogênio da região de defeito do nanotubo. O fato das energias de ligação serem muito próximas, indica que a superfície de potencial do sistema possui mínimos rasos.

Devido à amônia exibir dissociação sem barreira faz dela outra candidata em potencial a ser detectada pelo sistema $N R$, como observado experimentalmente por H. Terrones et

\footnotetext{
${ }^{2}$ Os cálculos citados foram realizados com o programa VASP que utiliza ondas planas. O fato de se usar esse tipo de conjunto de funções base elimina a necessidade de se fazer a correção de erro BSSE.
} 
al. [33] e corroborado teoricamente pelo trabalho realizado em nosso grupo [36].

\subsubsection{Resumo dos resultados do estudo do processo de disso- ciação}

Como dito anteriormente, devido à dificuldade de se obter as superfícies de potencial dos sistemas, adotamos o procedimento de amostragem, escolhendo configurações que julgamos razoáveis para o estudo do processo de dissociação das moléculas. Na Tab. 4.2 estão mostradas as energias de ligação $E_{b}$ para todas as estruturas apresentadas neste trabalho.

Os resultados obtidos até aqui permite-nos dividir as moléculas estudadas em três grupos, conforme Tab. 4.3:

Tabela 4.3: Resumo dos resultados obtidos do estudo do processo de dissociação.

\begin{tabular}{llc}
\hline \hline & O que ocorre? & moléculas \\
\hline Grupo 1 & Pode dissociar (possivelmente com barreira) & $\mathrm{H}_{2} \mathrm{O}, \mathrm{H}_{2} \mathrm{~S}_{\mathrm{O}} \mathrm{O}_{2}$ e $\mathrm{H}_{2}$ \\
& e algumas estruturas se ligaram na região de defeito & \\
Grupo 2 & Não dissocia ou liga na região de defeito & $\mathrm{CO}_{2}$ e $\mathrm{N}_{2}$ \\
Grupo 3 & A molécula se dissocia/liga sem barreira de potencial & $\mathrm{CO}$ e $\mathrm{NH}_{3}$ \\
\hline
\end{tabular}

Em relação ao Grupo 1, os resultados apresentados mostraram que as moléculas poderiam se dissociar, uma vez que a energia da estrutura dissociada é menor quando comparada com a soma das energias dos sub-sistemas isolados. Entretanto, os resultados são insuficientes para inferir qual seria a probabilidade que cada uma dessas espécies teria para se dissociar, já que não conhecemos a superfície de potencial e nem a barreira de potencial a ser vencida.

Para o Grupo 2, concluímos que as moléculas $\mathrm{CO}_{2}$ e $\mathrm{N}_{2}$ não se ligam na região do defeito do nanotubo uma vez que apresentaram energias de ligação positivas para todos os casos estudados. Em relação ao $N_{2}$ é um resultado interessante, já que é o principal constituinte do ar atmosférico, o que em princípio poderia interferir na detecção de outros gases.

Finalmente, no Grupo 3, temos as moléculas que se ligaram ou dissociaram sem barreira de potencial. Para o $C O$, em todos os casos testados, a molécula se ligou da mesma 
maneira e o processo ocorre sem barreira, com energia de ligação de $-0.41 e V$. Para o $\mathrm{NH}_{3}$, duas configurações apresentaram dissociação, sendo uma mais estável (Fig. 4.10(a)) com energia de ligação de $-0.59 \mathrm{eV}$. Essas duas espécies são as que apresentam maior potencial para serem detectadas pelo sistema $N R$.

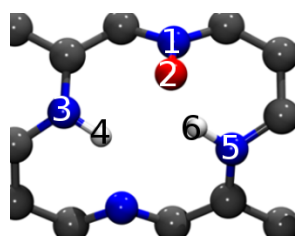

(a) $\mathrm{H}_{2} \mathrm{O}(a)$

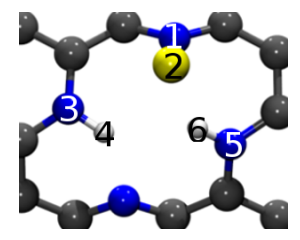

(e) $\mathrm{H}_{2} S(a)$

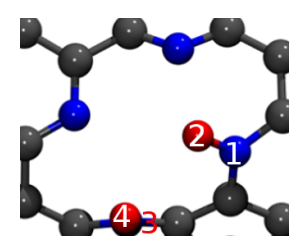

(b) $\mathrm{O}_{2}(a)$

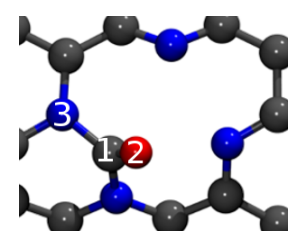

(f) $\mathrm{CO}$

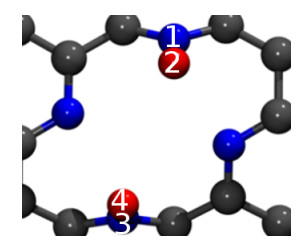

(c) $\mathrm{O}_{2}(b)$

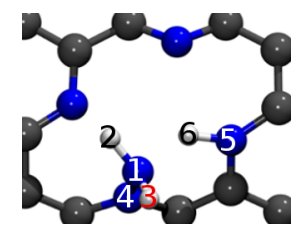

(g) $\mathrm{NH}_{3}(a)$

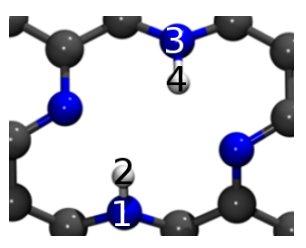

(d) $\mathrm{H}_{2}$

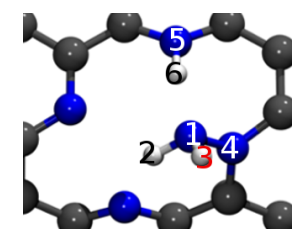

(h) $\mathrm{NH}_{3}(b)$

Figura 4.11: Figuras esquemáticas com os rótulos dos átomos de cada estrutura dissociada que se relaciona com os dados da Tab. 4.4.

A Tab. 4.4 mostra os comprimentos de ligação entre os átomos da molécula e os átomos da região de defeito e também para algumas ligações entre átomos do defeito que estão mais próximos à molécula; os números dos átomos estão relacionados com os rótulos mostrados na Fig. 4.11.

Tabela 4.4: Comprimentos de ligações entre os átomos da molécula e os átomos da região de defeito.

\begin{tabular}{ccccccccc}
\hline Átomos & \multicolumn{7}{c}{ Comprimento de ligação $(\AA)$} \\
& $\mathrm{H}_{2} \mathrm{O}(\mathrm{A})$ & $\mathrm{O}_{2}(a)$ & $\mathrm{O}_{2}(b)$ & $\mathrm{H}_{2}$ & $\mathrm{H}_{2} S(a)$ & $\mathrm{CO}$ & $\mathrm{NH}_{3}(a)$ & $\mathrm{NH} H_{3}(b)$ \\
\hline $1-2$ & 1.33 & 1.27 & 1.27 & 1.04 & 1.76 & 1.21 & 1.04 & 1.05 \\
$1-3$ & - & - & - & - & - & 1.47 & 1.03 & 1.03 \\
$1-4$ & - & - & - & - & - & 1.45 & 1.42 & 1.41 \\
$3-4$ & 1.03 & 1.26 & 1.27 & 1.03 & 1.04 & - & & - \\
$5-6$ & 1.08 & - & - & - & 1.07 & - & 1.07 & 1.04 \\
\hline \hline
\end{tabular}

A idéia principal por trás da possibilidade de nanoestruturas tais como nanotubos de carbono poderem ser usados como sensores de gás, está no fato de que uma pequena modificação em sua estrutura eletrônica, por exemplo devido à adsorção de uma molécula na superfície do nanotubo, poderia causar variações significativas em suas propriedades 
de transporte. Trabalhos teóricos através de cálculos de estrutura eletrônica de primeiros princípios indicam que as propriedades eletrônicas desses sistemas são modificadas mais significativamente quando há uma maior transferência de carga entre a molécula e o nanotubo. Desta forma, as moléculas que se dissociariam na superfície do nanotubo seriam mais facilmente detectadas (devido à maior transferência de carga) quando comparadas com as moléculas fisisorvidas [23, 24, 27, 31].

A Tab. 4.5 mostra a carga líquida $\left(Q_{\text {líquida }}\right)$ que os átomos de nitrogênio da região do defeito (correspondência com os rótulos na Fig. 4.2) apresentaram após o processo de ligação/dissociação das moléculas. Definimos a carga líquida do átomo $A$ por:

$$
Q_{\text {líquida }}^{A}=Q_{N R}^{A}-Q_{N R+\text { molécula }}^{A}
$$

em que $Q_{N R}^{A}$ é a carga (em unidades de $|e|$ ) do átomo $A$ antes da adsorção da molécula e $Q_{N R+\text { molécula }}^{A}$ é a carga do átomo $A$ depois da adsorção da molécula. Para as moléculas

Tabela 4.5: Análise populacional de Mulliken. Carga líquida (em unidades de $|e|$ ) em cada nitrogênio da região de defeito após o processo de ligação/dissociação. Os rótulos dos átomos de nitrogênio são os mesmos da Fig. 4.2.

\begin{tabular}{ccccccccccc}
\hline \hline átomo & $\mathrm{H}_{2} \mathrm{O}(\mathrm{a})$ & $\mathrm{H}_{2} \mathrm{O}(\mathrm{b})$ & $\mathrm{O}_{2}(\mathrm{a})$ & $\mathrm{O}_{2}(\mathrm{~b})$ & $\mathrm{H}_{2}$ & $\mathrm{CO}$ & $\mathrm{NH}_{3}(\mathrm{a})$ & $\mathrm{NH}_{3}(\mathrm{~b})$ & $\mathrm{H}_{2} \mathrm{~S}(\mathrm{a})$ & $\mathrm{H}_{2} \mathrm{~S}(\mathrm{~b})$ \\
\hline $\mathrm{N}(1)$ & -0.065 & -0.017 & 0.149 & 0.141 & 0.327 & 0.211 & 0.147 & -0.055 & -0.069 & -0.010 \\
$\mathrm{~N}(2)$ & 0.280 & 0.040 & 0.020 & 0.122 & -0.084 & 0.008 & 0.284 & 0.064 & 0.267 & 0.036 \\
$\mathrm{~N}\left(1^{\prime}\right)$ & 0.163 & -0.011 & 0.151 & 0.015 & 0.327 & -0.025 & 0.141 & 0.313 & -0.041 & 0.013 \\
$\mathrm{~N}\left(2^{\prime}\right)$ & 0.286 & 0.028 & 0.018 & -0.002 & -0.084 & 0.200 & 0.004 & -0.042 & 0.282 & 0.014 \\
\hline
\end{tabular}

$\mathrm{H}_{2} \mathrm{O}$ e $\mathrm{H}_{2} \mathrm{~S}$, mostramos o caso dissociado e um caso em que a molécula está ligada por meio de ligação de hidrogênio. Para as demais moléculas, mostramos os casos que apresentaram dissociação. Por exemplo, para a estrutura final da molécula de $C O$ mostrada na Fig. 4.11(f) observamos que os nitrogênios rotulados por $\mathrm{N}(1)$ e $\mathrm{N}(2$ ') (que são os que participam da ligação com o carbono da molécula) adiquirem uma carga líquida de 0.211|e| e $0.200|e|$, respectivamente. Em outras palavras, esses átomos doam elétrons para o carbono da molécula. Para as outras estruturas dissociadas, também há maior transferência de carga entre os átomos que participam das ligações com os átomos da molécula. Quando comparamos os casos dissociados com os fisisorvidos, por exemplo $\mathrm{H}_{2} \mathrm{~S}(\mathrm{a})$ e $\mathrm{H}_{2} \mathrm{~S}(b)$, vemos que a transferência de carga é $\sim 10$ vezes maior no caso dissociado. 


\section{Capítulo 5}

\section{Propriedades de transporte eletrônico}

Os cálculos das propriedades de transporte consistem em investigar como a interação e transferências de cargas entre as moléculas e o nanotubo (devido ao processo de ligação/dissociação) modificam a estrutura eletrônica do sistema e consequentemente a probabilidade dos elétrons serem transmitidos. Esse estudo fundamenta-se no fato de que, em princípio, diferentes espécies químicas perturbam a região do defeito de diferentes maneiras resultando em propriedades de transporte características de cada sistema. Desta forma, espera-se que seja possível identificar a molécula que está ligada ao nanotubo analisando a condutância daquele sistema.

Partindo dos resultados obtidos no estudo do processo de dissociação (seção 4.1), foram feitos cálculos das propriedades de transporte dos sistemas $(N R+$ molécula $)$ para aquelas espécies que poderiam ser detectadas pelo nanotubo com defeito, conforme metodologia descrita na seção 3.2. Como as moléculas de $\mathrm{CO}_{2}$ e $\mathrm{N}_{2}$ apresentaram energias de ligação positiva e, portanto, não se ligariam à região de defeito do sistema $N R$, não foram investigadas as suas propriedades de transporte. Como dito no final da seção 4.1.3, espera-se que sistemas em que a molécula esteja distante do nanotubo não apresentem mudanças significativas em suas propriedades de transporte, uma vez que praticamente não ocorre transferência de carga entre a molécula e o nanotubo. Por essa razão, seria interessante realizar os cálculos apenas para os casos em que a molécula está ligada/dissociada na região de defeito. Entretanto, vimos que para as moléculas de $\mathrm{H}_{2} \mathrm{O}, \mathrm{H}_{2} \mathrm{~S}$ e $\mathrm{NH}_{3}$, algumas estruturas, apesar de não se dissociarem, ficam ligadas através de ligações de hidrogênio. 


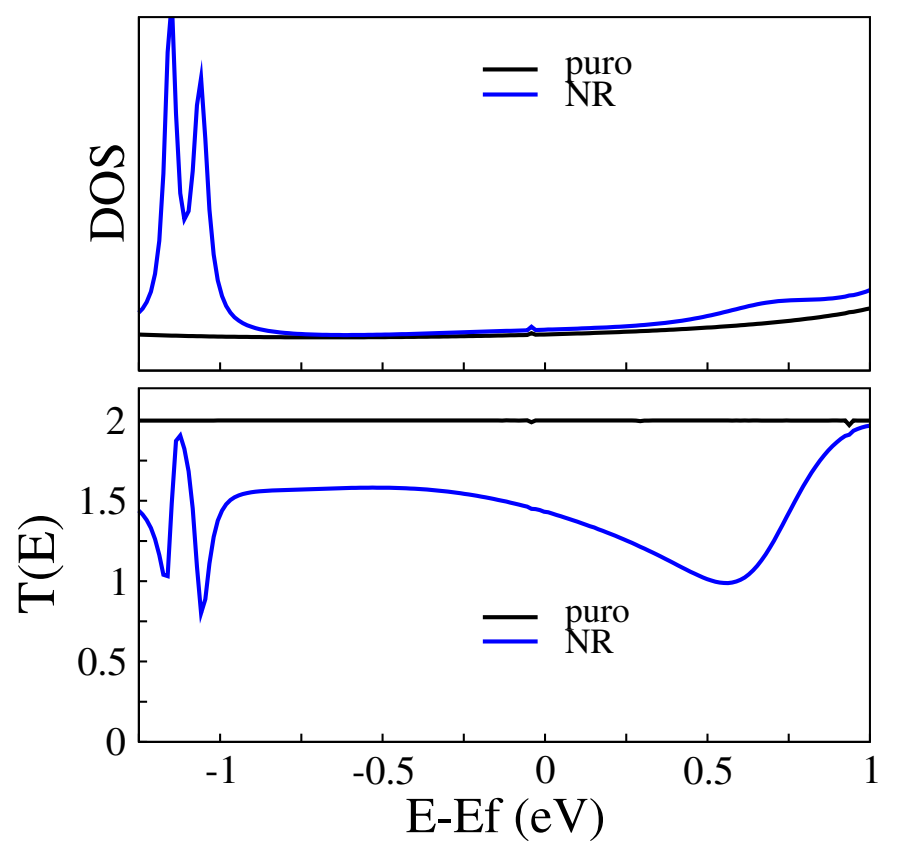

Figura 5.1: Propriedades de transporte do nanotubo com defeito $(N R)$ e do nanotubo puro. O painel superior(inferior) mostra a DOS(Transmitância) em função da energia, calculadas com o formalismo das funções de Green.

Portanto, também foram feitos cálculos das propriedades de transposte para algumas destas estruturas em que a molécula está distante do nanotubo, para verificar se, nestas condições, elas poderiam ser detectadas.

O nanotubo $(5,5)$ é metálico com 2 sub-bandas passando pelo nível de Fermi (seção 2.3). Desta forma, a transmissão através deste sistema na região do nível de Fermi é igual a $2 G_{0}$, já que cada sub-banda de um condutor balístico ideal apresenta probabilidade de transmissão igual a 1. Devido aos defeitos criados no nanotubo, a transmitância diminui por causa do espalhamento sofrido pelos elétrons na região do defeito. A Fig. 5.1 compara a DOS e a transmitância em função da energia para o nanotubo com defeito e para o nanotubo puro. No intervalo de energia mostrado, a transmitância do nanotubo com defeito diminui e as maiores variações ocorrem para energias $\simeq-1 \mathrm{eV}$ em relação ao nível de Fermi e para energias $\simeq 0.5 \mathrm{eV}$ acima do nível de Fermi. Essas variações estão associadas aos picos na DOS para o mesmo intervalo de energia.

Para exemplificar a discussão sobre a diferença dos cálculos de PDOS utilizando o formalismo NEGF e condições periódicas de contorno feita no final da seção 3.2.2, a Fig. 5.2 mostra o cálculo (SIESTA) da densidade de estados projetada (PDOS) sobre os 4 


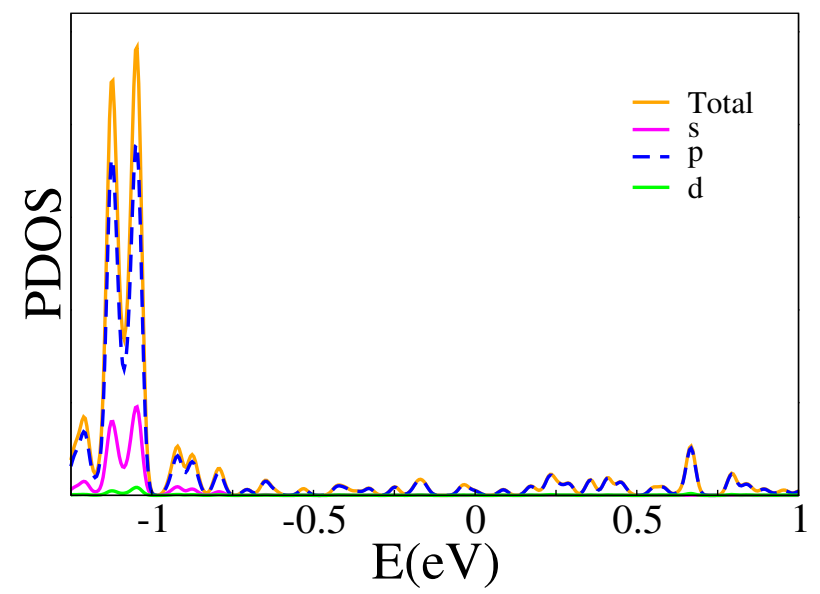

Figura 5.2: Densidade de estados projetada (PDOS) em função da energia sobre os 4 átomos de nitrogênio do nanototubo com defeito.

átomos de nitrogênio do nanotubo com defeito. Nessa abordagem, o sistema é resolvido considerando condições periódicas de contorno. Pode-se observar que há o aparecimento de mini-gaps [61], o que não acontece quando utilizamos o formalismo das funções de Green. Isso porque o sistema é aberto e resolvido para um único defeito, enquanto que no SIESTA o sistema é resolvido como se fosse infinito. Contudo, o que é importante, é verificarmos que os picos na DOS para energias $\simeq-1 e V$ abaixo do nível de Fermi (Fig. 5.1), são aqueles relacionados com as contribuições principalmente dos orbitais "p" dos átomos de nitrogênio da região de defeito do nanotubo (Fig. 5.2). Quando fazemos a PDOS apenas sobre um dos átomos de nitrogênio, vemos que a contribuição para a curva de PDOS deste átomo é praticamente a mesma para qualquer dos 4 átomos de nitrogênio. Por essa razão é que a Fig. 5.2 mostra a PDOS sobre os 4 átomos de uma só vez.

A Fig. 5.3 mostra a isosuperfície do cálculo da densidade de carga (LDOS) avaliada no intervalo de energia entre $-1.25 \mathrm{eV}$ e $-1 \mathrm{eV}$, associada aos picos na DOS (Fig. 5.1), que por sua vez estão associados, principalmente, aos orbitais "p" dos átomos de nitrogênio do nanotubo com defeito. Como dito anteriormente, é na região do defeito que estudaremos a adsorção de moléculas e, portanto, faz-se necessário verificar como a interação da molécula com o nanotubo modifica a estrutura eletrônica desta região. 

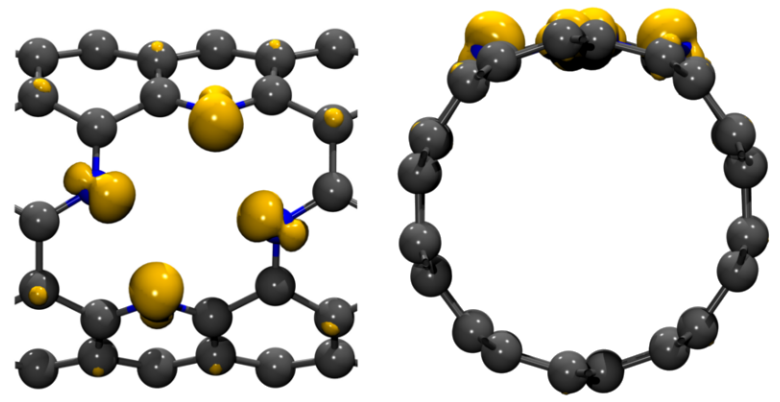

Figura 5.3: LDOS, para o intervalo de energia entre $-1.25 \mathrm{eV}$ e $-1.0 \mathrm{eV}$ do gráfico de DOS mostrado na Fig. 5.1. O valor considerado para a isosuperfície foi de $0.075 e / B o h r^{3}$. As duas imagens correspondem à mesma figura vista de diferentes maneiras.

\subsection{Resultados individuais para os sistemas nanotubo+ molécula}

\subsection{1 Água $\left(\mathrm{H}_{2} \mathrm{O}\right)$}

Para a molécula de $\mathrm{H}_{2} \mathrm{O}$ realizamos os cálculos das propriedades de transporte das estruturas finais mostradas nas Fig. 4.3(a) e Fig. 4.3(b). Os resultados de transmitância, densidade de estados total (DOS), a densidade de estados projetada (PDOS) em função da energia para as duas estruturas estão mostrados na Fig. 5.4.

Os gráficos do painel esquerdo indicam que a perturbação causada pela molécula de água dissociada na região do defeito modifica significativamente a transmitância do nanotubo. No nível de Fermi, praticamente não há alteração no valor da transmitância. Entretanto, para energias iguais a $-0.5 \mathrm{eV}$ e $0.5 \mathrm{eV}$ em relação ao nível de Fermi, podemos observar que a transmitância sofre um decréscimo de $38 \%$ e um aumento de $78 \%$, respectivamente. Essa variação está relacionada com mudanças na DOS. Mesmo que essas mudanças sejam pequenas, vemos que para intervalos de energia em que há um aumento(diminuição) na transmitância, há um decréscimo(aumento) na densidade de estados do sistema molécula $+N R$ quando comparada com a DOS do $N R$. Para energias abaixo de $-1 e V$ em relação ao nível de Fermi, pode-se verificar que a presença dos átomos da molécula removem os estados localizados que vêm principalmente da contribuição dos orbitais "p" dos átomos de nitrogênios do nanotubo, o que leva ao consequente aumento da transmitância nessa faixa de energia. A remoção desses estados localizados para en- 

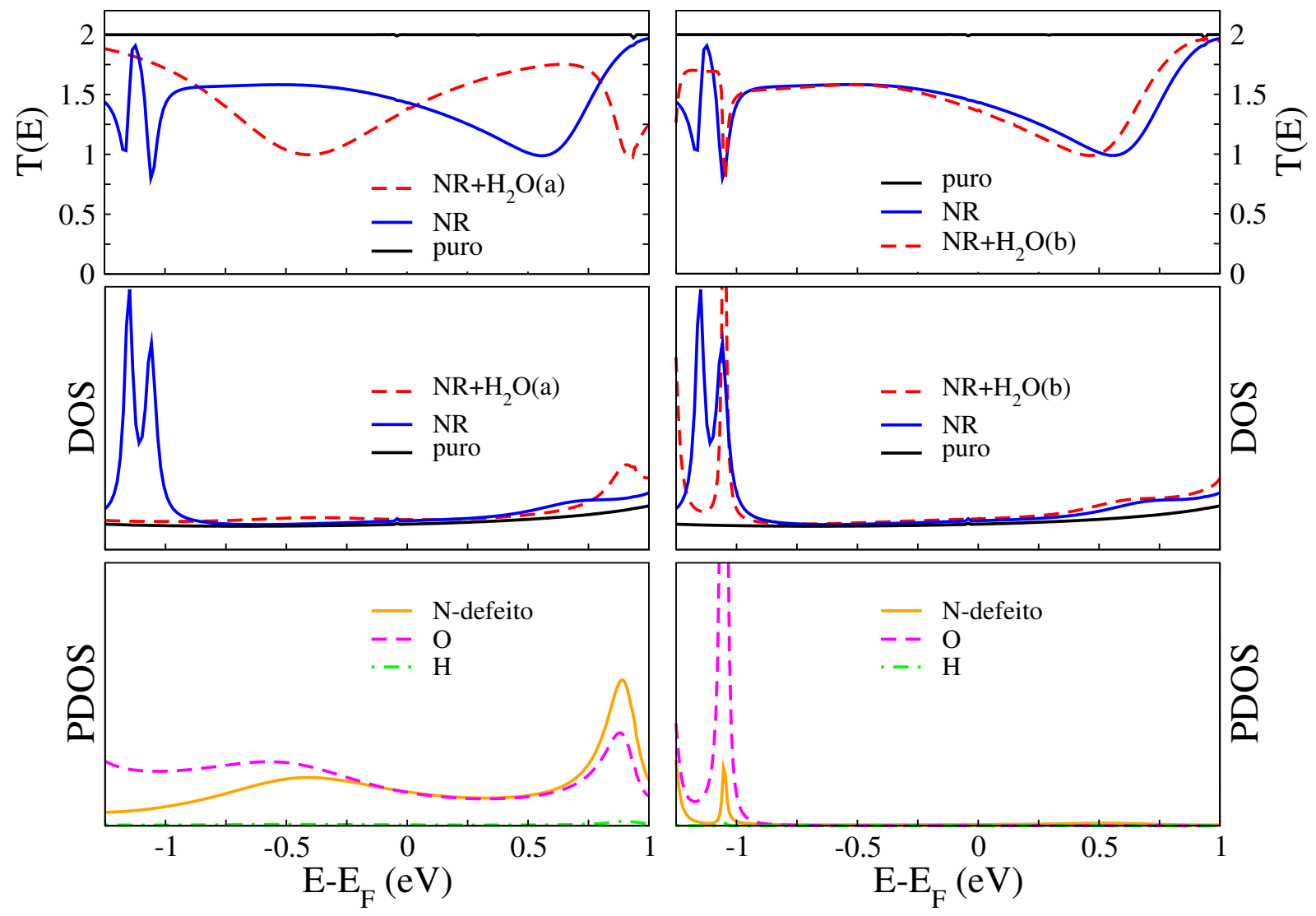

Figura 5.4: Painéis esquerdo e direito: propriedades de transporte referentes às estruturas mostradas nas Fig. 4.3(a) e Fig. 4.3(b), respectivamente. Painéis: superior, intermediário e inferior são respectivamente, Transmitância, DOS e PDOS em função da energia. Em particular, para a PDOS, as curvas se referem apenas às estruturas $N R+$ molécula.

ergias em torno de $-1.0 \mathrm{eV}$ está associada à saturação dos átomos de nitrogênio pelos átomos da molécula, como pode ser visto pelos resultados do cálculo da densidade de carga mostrado na Fig. 5.5.

Para a estrutura adsorvida ao nanotubo, os gráficos do painel da direita mostram que a transmitância não sofre alterações significativas e os estados devido às contribuições dos nitrogênios continuam bem localizados, conforme as curvas de DOS e PDOS. Isso ocorre porque a transferência de cargas entre os átomos da molécula e os átomos do nanotubo (nesse caso os nitrogênios) é pequena quando comparada à do sistema dissociado. Isto também pode ser notado na Tab. 4.5 indicando que a ligação de hidrogênio causa apenas uma pequena alteração na estrutura eletrônica do nanotubo. 


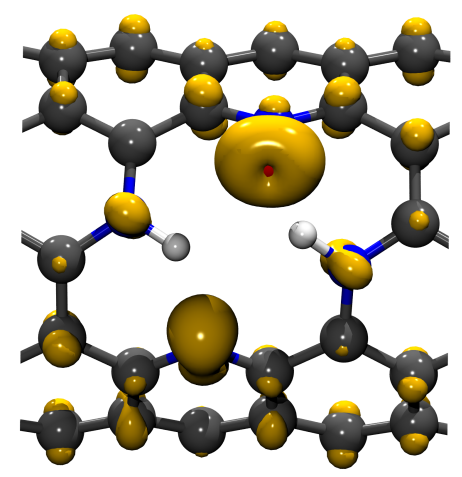

Figura 5.5: LDOS, para o intervalo de energia de $-1.25 \mathrm{eV}$ a $-0.75 \mathrm{eV}$ do gráfico de DOS mostrado na Fig. 5.4. O valor considerado para a isosuperfície foi de $0.035 e / B o h r^{3}$.

\subsection{2 Ácido Sulfídrico $\left(H_{2} S\right)$}
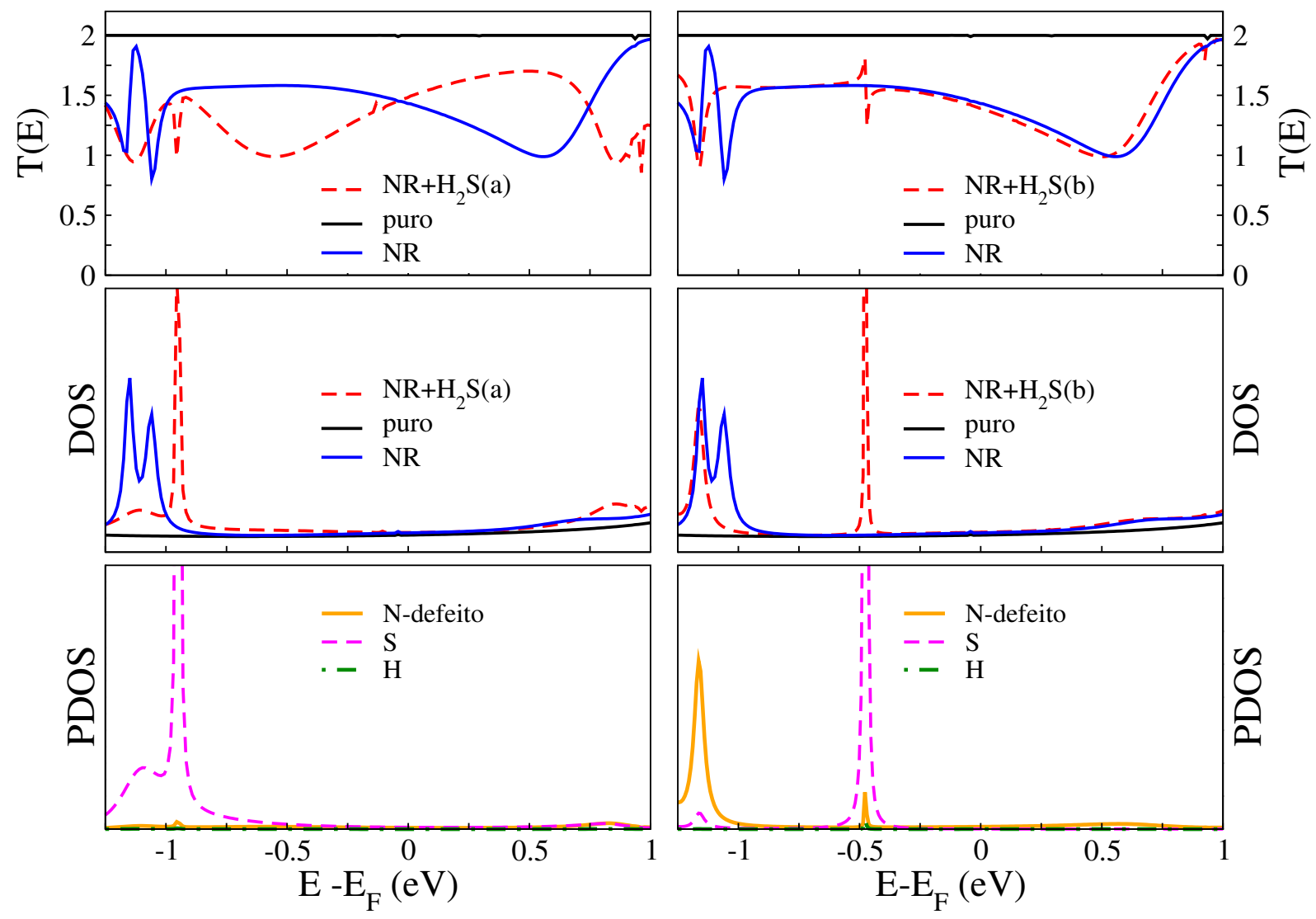

Figura 5.6: Painéis esquerdo e direito: propriedades de transporte referentes às estruturas mostradas nas Fig. 4.7(a) e Fig. 4.7(b), respectivamente. Painéis: superior, intermediário e inferior são respectivamente, PDOS, DOS e Transmitância em função da energia. As curvas de PDOS se referem apenas às estruturas $N R+$ molécula. 


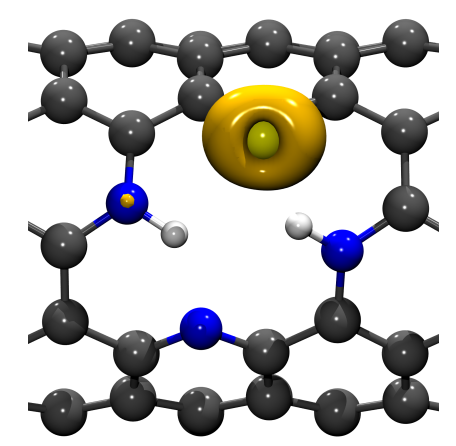

Figura 5.7: LDOS calculada em torno de $-1 e V$ com relação ao nível de Fermi para a estrutura $\mathrm{H}_{2} \mathrm{~S}$ dissociada. O valor considerado para a isosuperfície fo de $0.035 e / \mathrm{Bohr}{ }^{3}$.

De forma análoga, para a molécula de $H_{2} S$ foram feitos cálculos das propriedades de transporte das duas estruturas finais mostradas nas Fig. 4.7(a) e Fig. 4.7(b) e os resultados estão apresentados na Fig. 5.8. Para a estrutura dissociada (painel da esquerda), observamos a mesma relação entre a transmitância e a DOS para o intervalo de energia $\pm 0.5 \mathrm{eV}$ em relação ao nível de Fermi quando comparados com o caso da água. Os extremos na curva de transmitância para as energias $-0.5 \mathrm{eV}$ e $0.5 \mathrm{eV}$ também são semelhantes com diminuição de $38 \%$ e aumento de $73 \%$, respectivamente. No entanto, diferentemente do caso da água, para energias $\leq-1 \mathrm{eV}$ surgem estados localizados devido à presença da molécula, em particular associados ao átomo de enxofre, de acordo com os resultados de PDOS (Fig. 5.8). A Fig. 5.7 mostra a densidade de carga calculada no intervalo de energia de $-1.25 \mathrm{eV}$ a $-0.75 \mathrm{eV}$ abaixo do nível de Fermi, a qual apresenta maior concentração no átomo de enxofre. Isso reflete o pico localizado devido a esse átomo, como visto nas curvas de PDOS. Quando olhamos para a densidade de carga da água dissociada (Fig. 5.5), para o mesmo intervalo de energia, vemos que é mais deslocalizada. Isso pode ser visto nas curvas de PDOS mostradas na Fig. 5.4, já que não aparecem picos intensos como no caso da molécula de $\mathrm{H}_{2} \mathrm{~S}$.

Para a estrutura não dissociada, a transmitância não sofre variações significativas, embora o átomo de enxofre dê origem a um estado localizado em $-0.5 \mathrm{eV}$, como mostrado nos gráficos do painel direito. 


\subsubsection{Oxigênio molecular $\left(O_{2}\right)$}

Para a molécula de $O_{2}$, duas estruturas finais apresentaram dissociação. Entretanto, como vimos no estudo do processo de dissociação, a estrutura apresentada na Fig. 4.5(a) possui energia de ligação $\simeq 0$ e, portanto, possivelmente não seria observada. Mas por motivos de comparação, foram feitos cálculos das propriedades de transporte apenas das duas estruturas dissociadas. Nos dois casos, os resultados mostram que a transmitância sofre mudanças significativas apenas para valores de energia em que a contribuição para a DOS, devido aos átomos de oxigênio da molécula, é maior do que a contribuição devido aos átomos de nitrogênio. Por exemplo, para o caso mostrado no painel esquerdo, o gráfico de PDOS indica que a contribuição dos átomos de oxigênio é maior que aquela devido aos átomos de nitrogênio em torno de $-0.75 \mathrm{eV}$ e isso provoca um aumento na
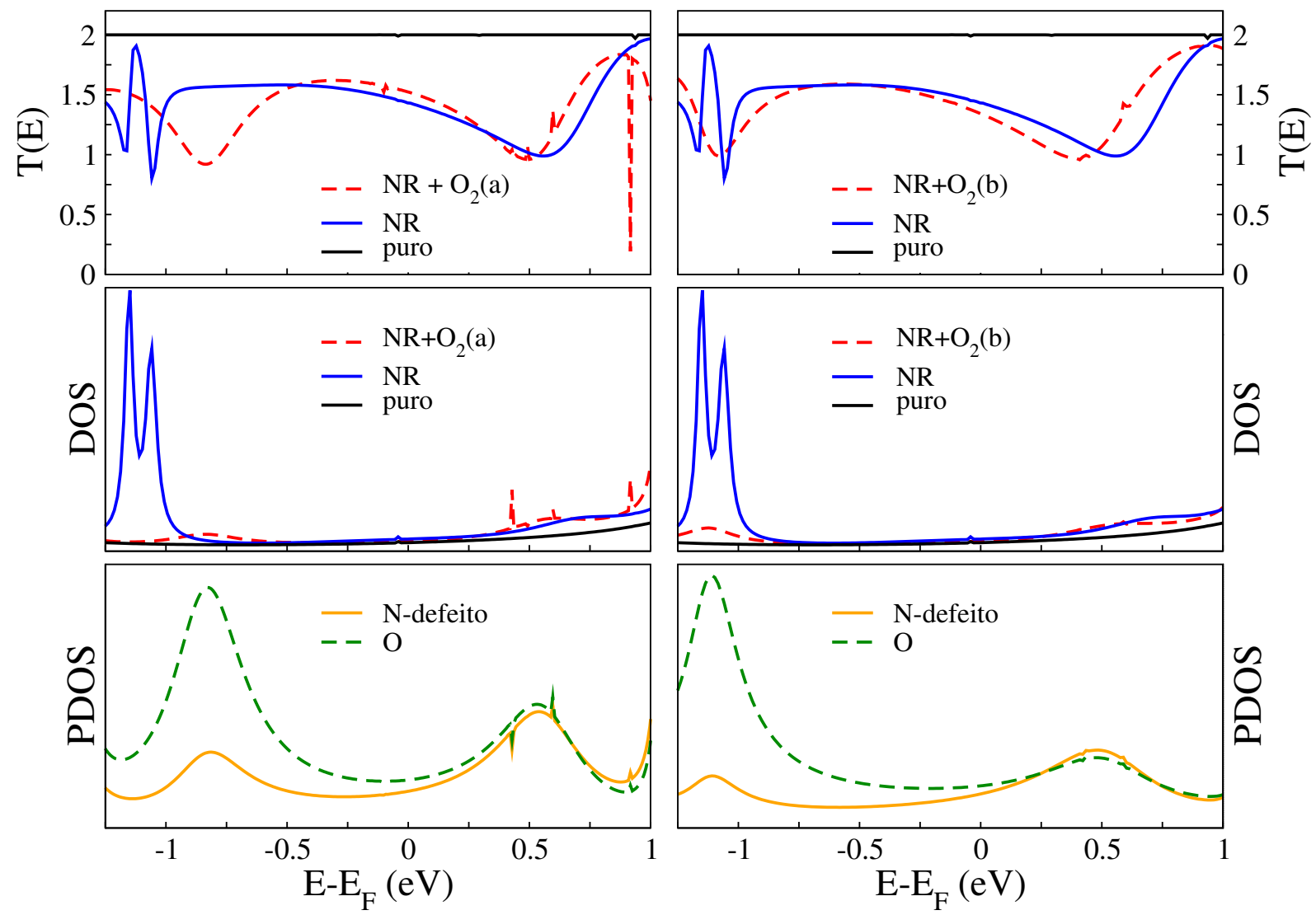

Figura 5.8: Painéis esquerdo e direito: Propriedades de transporte referentes às estruturas mostradas nas Fig. 4.5(a) e Fig. 4.5(b), respectivamente. Painéis: superior, intermediário e inferior são respectivamente, PDOS, DOS e Transmitância em função da energia. As curvas de PDOS se referem apenas às estruturas $N R+$ molécula. 
DOS para o mesmo intervalo de energia, o que consequentemente causa a diminuição da transmitância. Para o caso dissociado mostrado no painel direito, observamos também mudanças expressivas na transmitância do sistema, principalmente na região de concentração de estados devido aos átomos de nitrogênio do defeito $(\simeq-1 e V)$. A energia de ligação calculada para essa estrutura foi de $-0.68 \mathrm{eV}$ e ela seria a mais possivelmente encontrada.

\subsubsection{Hidrogênio $\left(H_{2}\right)$}

A dissociação da molécula de $H_{2}$ ocorreu em apenas um dos casos estudados, conforme mostrado na Fig. 4.6(a). Para esse sistema foram feitos cálculos das propriedades

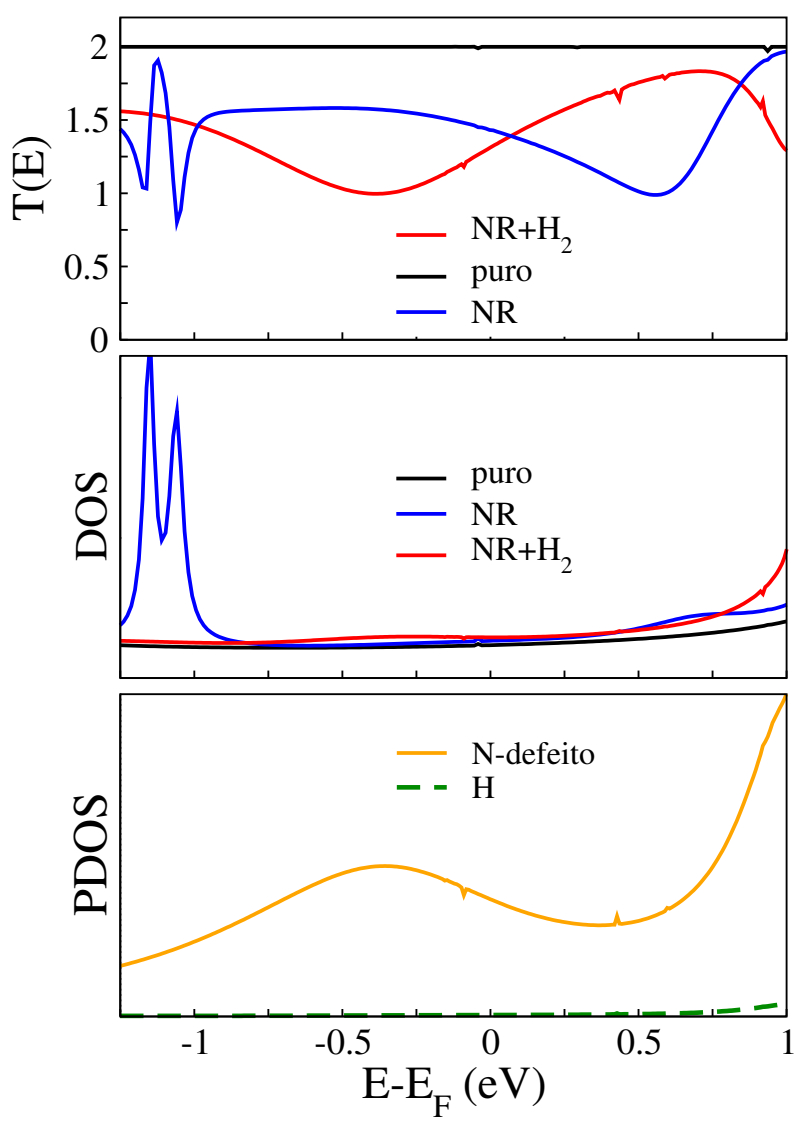

Figura 5.9: Propriedades de transporte do $\mathrm{H}_{2}$ (Fig. 4.6(a)). Painéis: superior, intermediário e inferior são respectivamente, PDOS, DOS e Transmitância em função da energia. As curvas de PDOS se referem apenas às estruturas $N R+$ molécula.

de transporte. Os resultados mostrados na Fig. 5.9 indicam que ocorrem mudanças significativas na transmitância e na DOS do nanotubo devido à presença da molécula 
dissociada. Mesmo que a contribuição para a DOS dos átomos de hidrogênio seja pequena quando comparada com a contribuição dos átomos de nitrogênio (ver PDOS), estes átomos removem a localização de estados em energias de $\sim-1 e V$ associados aos nitrogênios do nanotubo. De maneira análoga aos resultados anteriores, em torno do nível de Fermi para valores de $\pm 0.5 \mathrm{eV}$, a transmitância sofre modificações significativas, o que em princípio resultaria em um sinal de corrente indicando a presença da molécula. Este resultado corrobora as observações das seções anteriores no sentido de que as mudanças nas propriedades de transporte estão essencialmente associadas à saturação dos átomos de nitrogênio do defeito.

\subsubsection{Monóxido de carbono $(C O)$}

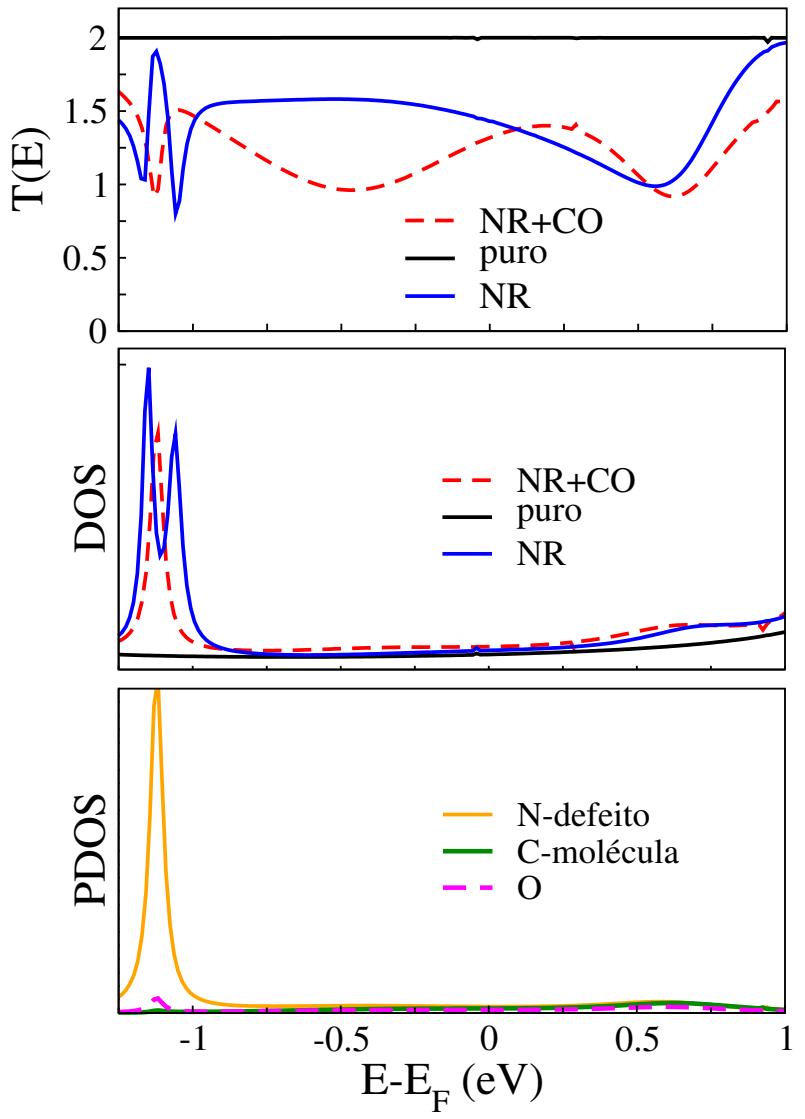

Figura 5.10: Propriedades de transporte do monóxido de carbono. Painéis: superior, intermediário e inferior são respectivamente, PDOS, DOS e Transmitância em função da energia. As curvas de PDOS se referem apenas às estruturas $N R+$ molécula.

Para a molécula de $C O$, foram realizados cálculos das propriedades de transporte 
da estrutura final obtida do processo de relaxação e os resultados estão apresentados na Fig. 5.10. A transmitância decai na região em torno de $\sim-0.5 \mathrm{eV}$ em relação ao nível de Fermi, mas sofre pequenas variações para valores de energia acima de $E_{F}$. Para energias abaixo de $-1 e V$ em relação ao nível de Fermi (onde se concentram os estados dos nitrogênios da região do defeito), a presença da molécula ainda mantém a localização desses estados, ao contrário do que foi observado, por exemplo, para a molécula de $\mathrm{H}_{2} \mathrm{O}$ e $\mathrm{H}_{2}$.

Como foi discutido na seção 4.1.2, o monóxido de carbono foi o único que se ligou, sem apresentar barreira de potencial, para todas as configurações testadas com energia de ligação igual a $-0.41 \mathrm{eV}$. Isso faz dele um candidato em potencial para ser detectado pelo sistema $N R$. De fato, os resultados das propriedades de transporte mostram que essa molécula poderia ser detectada, já que as propriedades de transporte do nanotubo com defeito é modificada.

\subsubsection{Amônia $\left(\mathrm{NH}_{3}\right)$}

Para a molécula de amônia, foram feitos cálculos das propriedades de transporte para as duas estruturas em que a molécula se dissociou (Fig. 4.10(a) e Fig. 4.10(b)) e os resultados estão apresentados na Fig. 5.11. A presença da molécula novamente remove os estados concentrados no intervalo de energia abaixo de $-1 e V$ em relação ao nível de Fermi, associados aos nitrogênios do defeito. A principal diferença entre os dois sistemas está na transmitância para energias acima do nível de Fermi. No primeiro caso, há um aumento na transmitância quando comparada com a mesma quantidade do nanotubo $N R$, enquanto que no segundo caso, ocorre uma diminuição em torno de $+0.75 \mathrm{eV}$.

Assim como ocorreu para o $\mathrm{CO}$, a dissociação do $\mathrm{NH}_{3}$ é feito sem barreira e, portanto, poderia ser detectado pelo sistema $N R$. Isso porque os resultados mostram que as propriedades de transporte do nanotubo com defeito são modificadas devido à presença da molécula. 

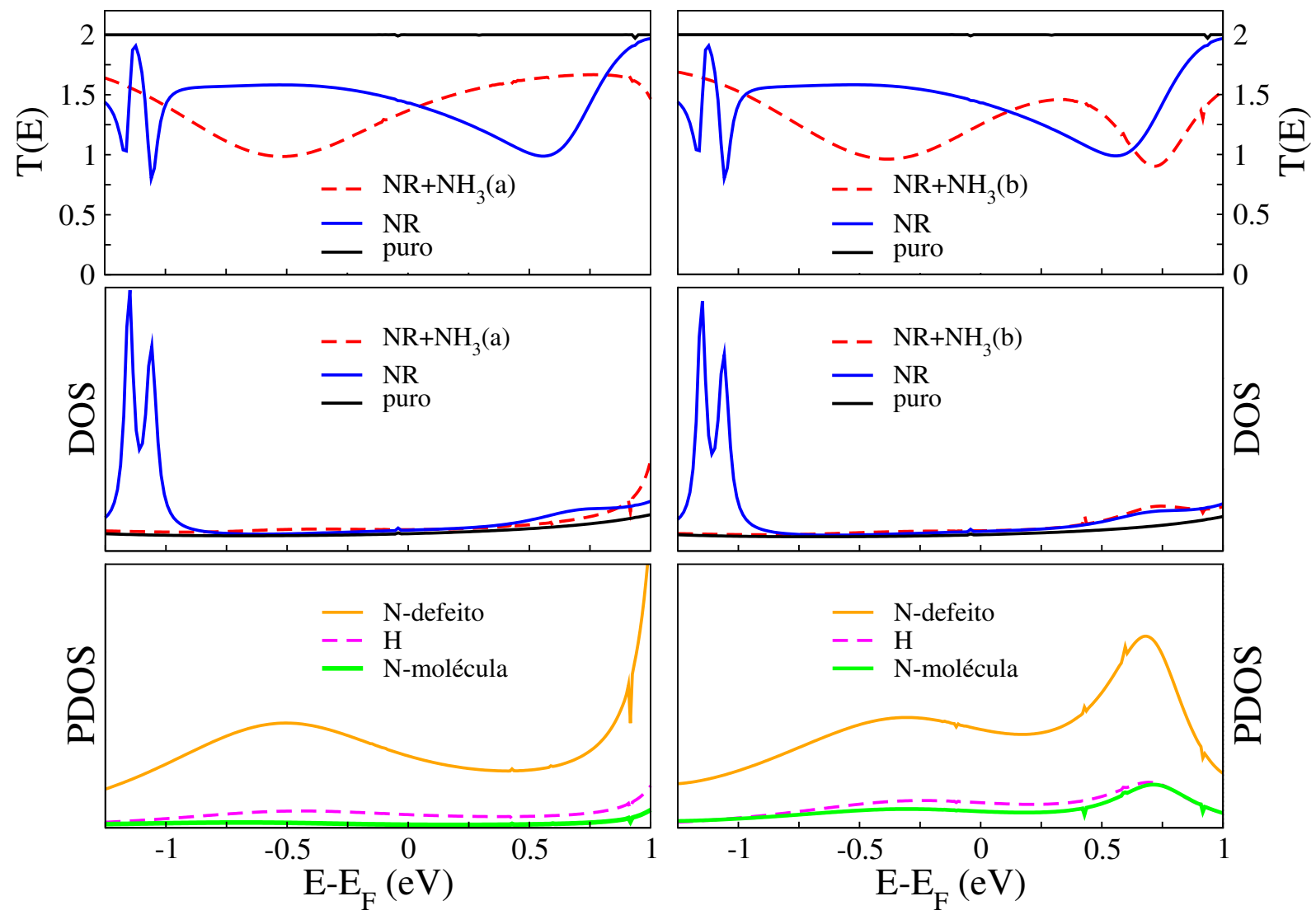

Figura 5.11: Painéis esquerdo e direito: propriedades de transporte referentes às estruturas mostradas nas Fig. 4.10(a) e Fig. 4.10(b), respectivamente. Painéis: superior, intermediário e inferior são respectivamente, Transmitância, DOS e PDOS em função da energia. As curvas de PDOS se referem apenas às estruturas $N R+$ molécula.

\subsection{Como tratar a seletividade?}

Uma característica interessante que se busca em um sensor de gás é a seletividade, que implica em não só detectar a molécula e fornecer uma possível concentração deste gás, mas ter a capacidade de distingui-la de outras espécies químicas quando o dispositivo é inserido em um ambiente contendo uma mistura de gases.

A Fig. 5.12 apresenta um resumo dos cálculos das propriedades de transporte para todas as estruturas que seriam possíveis de se detectar. Deve-se observar que as mudanças nas curvas de transmitância para as diferentes moléculas estudadas são muito parecidas (exceto a molécula de $O_{2}$ ) e até mesmo entre os cálculos feitos para diferentes configurações da mesma molécula. Entretanto, o que é experimentalmente observado é a corrente que o sistema apresenta em resposta a uma diferença de potencial externa aplicada. No limite 
de voltagens nulas, podemos calcular a condutância no regime linear dada pela Eq. 3.15 e a corrente será dada pela integração da curva de transmitância em relação à energia para energias em torno do nível de Fermi.
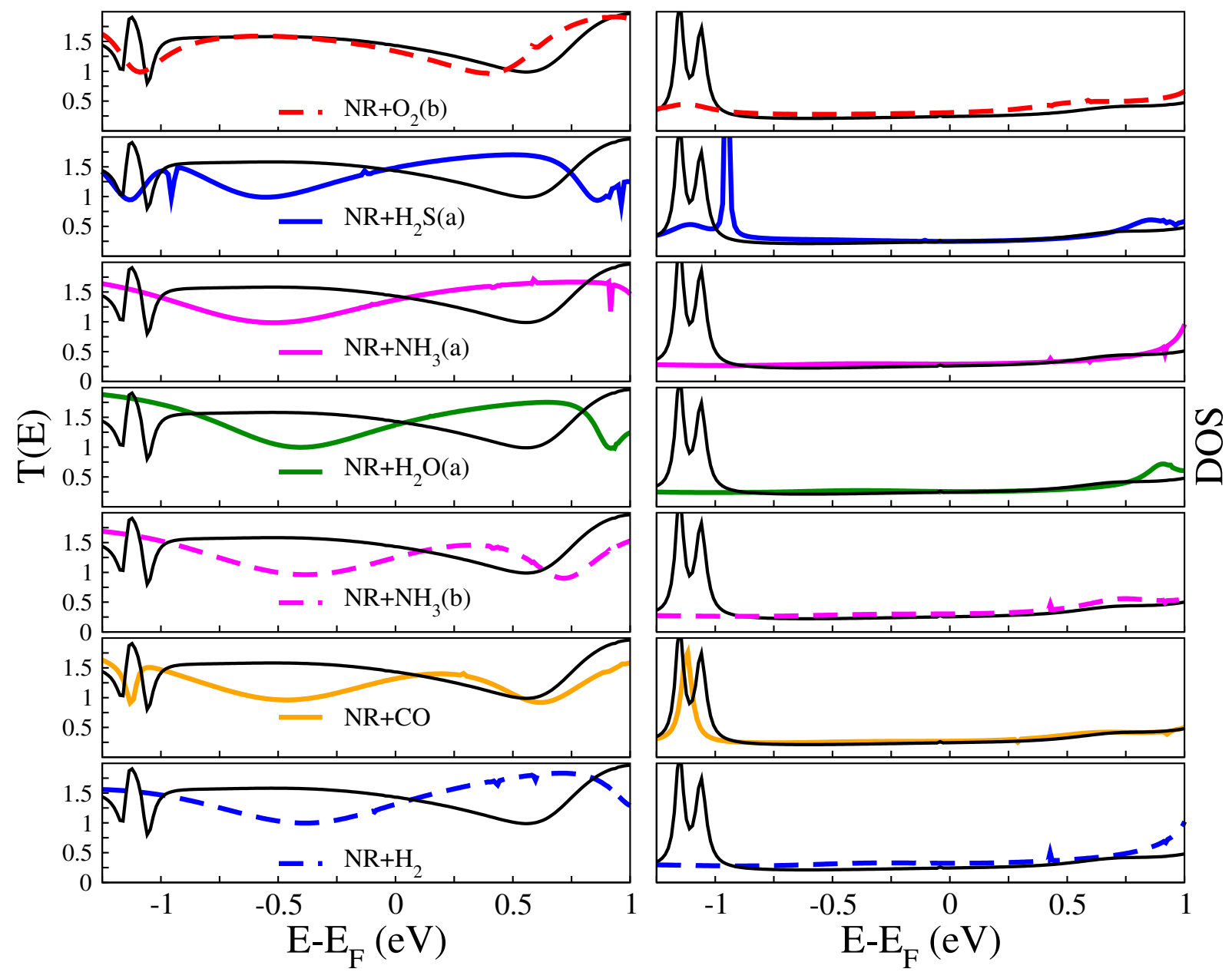

Figura 5.12: Transmitâncias e DOS de todas as estruturas ligadas/dissociadas que poderiam ser detectadas pelo sistema $N R$.

Definimos a variação percentual $\left(\Delta T\left(E_{F}\right)\right)$ da transmitância de cada sistema $(N R+$ molécula) calculada no nível de Fermi, em relação à transmitância também calculada no nível de Fermi para o sistema $N R$ por:

$$
\Delta T\left(E_{F}\right)=\left\{\frac{T\left(E_{F}\right)_{N R+\text { molecula }}}{T\left(E_{F}\right)_{N R}} \times 100 \%\right\}-100 \% .
$$

Também definimos a variação percentual da corrente de cada sistema $(N R+$ molécula $)$ em relação à corrente do nanotubo $N R$, calculadas no regime linear, por:

$$
\Delta I_{\text {Regime-linear }}=\left\{\frac{I_{N R+\text { molecula }}}{I_{N R}} \times 100 \%\right\}-100 \%,
$$


em que,

$$
I=\int_{-0.025}^{0.025} \frac{2 e}{h} T(E) d E .
$$

Na Tab. 5.1 estão apresentadas essas variações percentuais calculadas a partir das Eqs. 5.1 e 5.2. As corrente calculadas pela Eq. 5.3 correspondem às áreas sob as curvas

Tabela 5.1: Variação percentual da transmitância calculada no nível de Fermi e variação percentual da corrente no regime linear para cada sistema $(N R+$ molécula $)$ em relação às mesmas quantidades para o nanotubo $N R$.

\begin{tabular}{ccc}
\hline \hline molécula & $\Delta T\left(E_{F}\right)$ & $\Delta I_{\text {Regime-linear }}$ \\
\hline $\mathrm{O}_{2}(b)$ & $-11.3 \%$ & $-11.1 \%$ \\
$\mathrm{H}_{2} \mathrm{~S}(a)$ & $-1.8 \%$ & $-3.9 \%$ \\
$\mathrm{H}_{2} \mathrm{O}(a)$ & $-9.1 \%$ & $-10.5 \%$ \\
$\mathrm{CO}$ & $-12.7 \%$ & $-12.5 \%$ \\
$\mathrm{H}_{2}$ & $-13.2 \%$ & $-13.4 \%$ \\
$\mathrm{NH}_{3}(a)$ & $-9.5 \%$ & $-10.8 \%$ \\
$\mathrm{NH}_{3}(b)$ & $-17.2 \%$ & $-18.5 \%$ \\
\hline \hline
\end{tabular}

de transmitância mostradas na Fig. 5.13 no intervalo de $-0.025 \mathrm{eV}$ a $0.025 \mathrm{eV}$. De forma geral, os cálculos de corrente no regime linear para todos os sistemas estudados nesse trabalho indicam uma diminuição no sinal devido à presença da molécula. Em outras palavras, quando analisamos a transmitância em torno do nível de Fermi, a presença da molécula reforça o espalhamento causado pela região de defeito do nanotubo, aumentando a resistência e diminuindo a corrente.

Pode-se observar que a estrutura que apresenta a maior variação da corrente é a $\mathrm{NH}_{3}(b)$ com $-18.5 \%$, de forma que seria a mais facilmente detectada em um experimento. Como visto anteriormente, ela apresentou duas configurações possíveis de dissociação. Porém, conforme Tab. 4.2, a mais estável corresponde à $\mathrm{NH}_{3}(a)$ que apresenta variação percentual de corrente de $\sim-10.8 \%$. Esta variação é muito próxima dos valores calculados para várias outras estruturas, como por exemplo, $\mathrm{O}_{2}(b), \mathrm{H}_{2} \mathrm{O}(a), \mathrm{CO}$, e $\mathrm{H}_{2}$; isso impossibilitaria determinar a espécie química que estaria ligada ao nanotubo, caso este estivesse inserido em um ambiente contendo uma mistura desses gases.

De forma análoga, a molécula de $O_{2}$ apresentou duas estruturas dissociadas; porém, apenas uma delas é estável à temperatura ambiente (Fig. 4.5(b)), com variação de energia 


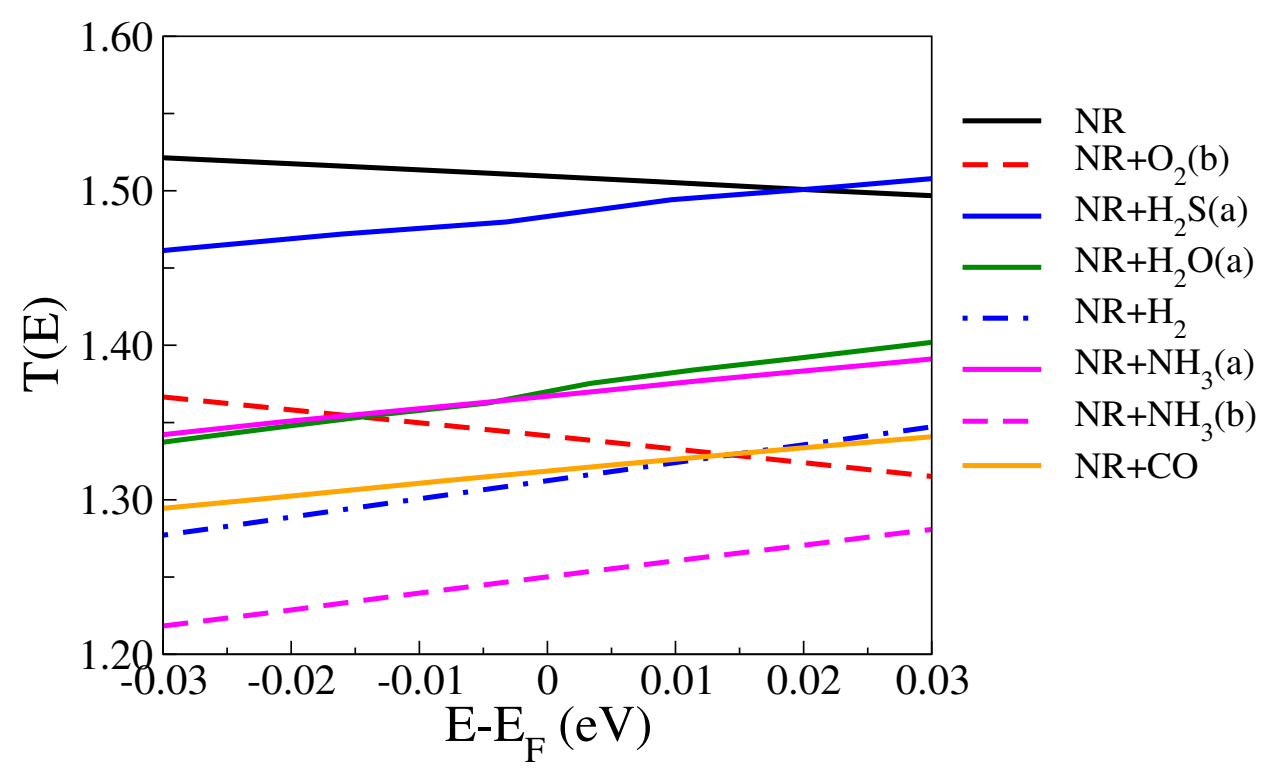

Figura 5.13: Transmitâncias das estruturas ligadas/dissociadas que poderiam ser detectadas pelo sistema $N R$ para energias em torno do nível de Fermi.

total de $-0.68 \mathrm{eV}$. No entanto, a variação percentual da corrente para essa estrutura foi de $-11.1 \%$, que é bem próxima daquelas mencionadas anteriormente. Em princípio, com $-3.9 \%$ de variação na corrente, apenas a molécula $H_{2} S(a)$ seria mais facilmente distinguida das demais. Todavia, faz-se necessário o conhecimento da superfície de potencial do sistema e a barreira de energia envolvida nos processos de dissociação para concluir se esta molécula ligaria ou não na região do defeito do nanotubo.

\subsection{Propriedades de transporte de sistemas desorde- nados}

Como já foi discutido, devido à baixa afinidade entre nanotubos puros e moléculas gasosas, faz-se necessária a funcionalização dos nanotubos para que os mesmos possam ser usados como sensores de gás. Os processos de síntese de nanotubos funcionalizados ainda não permitem a produção de nanotubos com defeitos especificamente posicionados, embora se tenha um bom controle da concentração destes defeitos (seção 2.4). Isso faz com que os defeitos estejam distribuídos aleatoriamente ao longo do comprimento do nanotubo, não apenas em distâncias, mas também devido à rotação relativa entre os defeitos. Desta maneira, seria importante levar em conta os fatores de desordem ao simular sensores de 
gás.

Em geral, a concentração em massa destes defeitos observada experimentalmente é $<4 \%$ [33, 66]. Para uma concentração de $2.6 \%$ de nitrogênio em relação ao número de átomos de carbono de um nanotubo $(5,5)$, a distância média entre os defeitos é de $22.4 \AA$ para o tipo de defeito $4 N$. Como o SIESTA utiliza condições periódicas de contorno, definimos uma caixa contendo 9 células unitárias do nanotubo $(5,5)$, conforme discutido no apêndice B. Isso corresponde a exatamente $22.4 \AA$ e essa distância, portanto, é a distância média entre os defeitos $4 N$.

O sistema desordenado é construído da seguinte maneira: para cada bloco com defeito (com ou sem molécula) e para blocos sem defeito (pristino) usados para a montagem do nanotubo desordenado (Fig. 3.5), fazemos um cálculo de estrutura eletrônica utilizando o código SIESTA para obtermos as matrizes hamiltoniano e "overlap" de cada um desses sistemas. Em uma situação real os defeitos nos nanotubos, além de estarem distribuídos ao longo do comprimento do mesmo, também podem estar rotacionados uns em relação aos outros. Para o nanotubo $(5,5)$, temos 5 rotações possíveis para que um bloco de defeito possa ser girado em relação ao outro, de forma que a quiralidade do nanotubo seja preservada. Assim, para cada estrutura de defeito (com ou sem molécula) e para cada rotação, fazemos os cálculos de estrutura eletrônica e armazenamos as matrizes hamiltoniano e "overlap" destes.

Para se controlar a concentração em massa dos defeitos de nitrogênio, consideramos também blocos de nanotubo puro (pristino) que são também distribuídos aleatoriamente entre os blocos de defeito. A distribuição aleatória de todos esses blocos é feita através de sorteios. Uma vez feito o sorteio de uma configuração e tendo as matrizes hamiltoniano e "overlap" de cada bloco, a matriz total do sistema é então construída conforme a Eq. 3.41 e os cálculos das propriedades de transporte do sistema desordenado segue a metodologia descrita na seção 3.2.3.

As simulações dos sistemas desordenados apresentadas nesse trabalho foram feitas com concentração de defeitos de $0.5 \%$ e $1.3 \%$, o que equivale a uma distância média entre os defeitos de $3 \mathrm{~nm}$ e $9 \mathrm{~nm}$, respectivamente. Como dito anteriormente, a concentração de defeitos na simulação é controlada, colocando-se unidades de nanotubos pristino entre blocos de defeito. Definindo o número de unidades de defeito e a concentração destes defeitos, fica definido o número de unidades pristino e, consequentemente, o comprimento 
do nanotubo desordenado (região de espalhamento), que nesse caso foi fixado em $220 \mathrm{~nm}$.

Além dos fatores de desordem mencionados anteriormente, também consideramos a desordem causada por diferentes concentrações das moléculas que se ligam às regiões de defeito, o que é feito da seguinte maneira: se a concentração de gás é $0 \%$, significa que todos os defeitos tipo $4 N$, que são distribuídos aleatoreamente, não possuem molécula ligada/dissociada; se a concentração de gás é 100\% significa que a simulação é feita com todos os defeitos ocupados pela molécula. Em concentrações intermediárias, os defeitos ocupados e os não ocupados pela molécula estarão distribuídos aleatoriamente ao longo do comprimento do nanotubo e também em relação às rotações relativas.

Os resultados do estudo do processo de dissociação das moléculas na região de defeito do nanotubo, apresentados na seção 4.1.3, indicam que as moléculas $\mathrm{CO}$ e $\mathrm{NH}_{3}$ seriam detectadas com mais facilidade quando comparadas com as demais, já que foram as únicas que o processo de ligação/dissociação é feito sem barreira. Ainda, dos cálculos de variação percentual de corrente no regime linear apresentados no final da seção 5.2, vimos que, apesar da presença da molécula causar variação na transmitância e consequentemente na corrente, essas variações são muito semelhantes para a maioria das moléculas, inclusive para $\mathrm{CO}$ e $\mathrm{NH}_{3}$. Diante desses resultados, baseado na metodologia apresentada na seção 3.2.3, foram feitos os cálculos das propriedades de transporte dos sistemas desordenados para o monóxido de carbono. Estes foram comparados com os resultados dos cálculos de desordem feitos para a amônia [36] em que a média da condutância é colocada em função da concentração de $\mathrm{NH}_{3}$. O objetivo desse estudo é investigar quais os efeitos de desordem sobre as propriedades de transporte dos sistemas e se esses efeitos podem ser usados para distinguir essas moléculas, em um caso em que o nanotubo estivesse inserido em um ambiente contendo uma mistura desses gases.

A Fig. 5.14 mostra os gráficos da média da condutância, em função da concentração de monóxido de carbono, para concentrações de defeitos iguais a $0.5 \%$ e $1.3 \%$. Para cada ponto do gráfico, foi feito uma média dos valores de condutância obtidos de aproximadamente 50 realizações ${ }^{1}$. Cada realização consiste de um sorteio dos blocos, ou seja, cada realização é um sistema diferente em que mantemos fixas as concentrações de defeito e do gás, bem como o comprimento do nanotubo. As barras de erros são os desvios padrão

\footnotetext{
${ }^{1}$ Esse número foi escolhido baseado em resultados de cálculos anteriores do grupo. Estes cálculos [36] foram feitos com aproximadamente 500 realizações. Através de testes, vimos que com 50 realizações, as médias e os desvios padrão já convergiam.
} 
relacionados à cada conjunto de 50 realizações. A média é necessária uma vez que, em

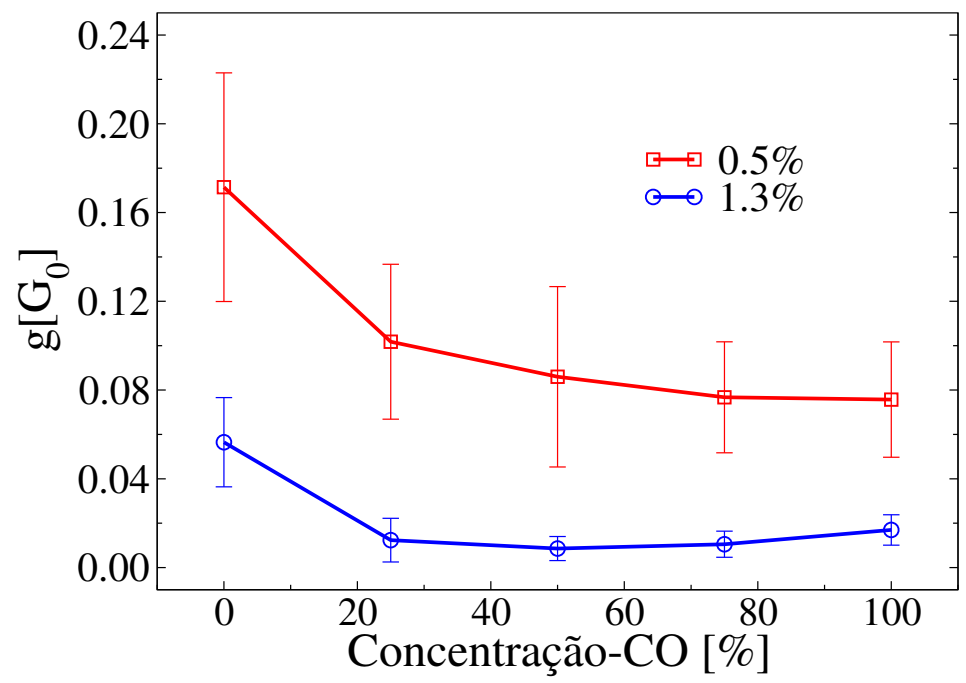

Figura 5.14: Média da condutância em função da concentração de $C O$ para concentrações de defeitos iguais a $0.5 \%$ e $1.3 \%$. O comprimento do nanotubo foi fixado em $220 \mathrm{~nm}$.

cada realização o sistema é diferente e a condutância final é diferente.

Pode-se observar que à medida que a concentração de monóxido de carbono aumenta, a média da condutância diminui, indicando que a presença da molécula reforça o espalhamento na região de defeito do nanotubo, conforme discutido para o caso do sistema isolado apresentado na Fig. 5.10. Considerando o gráfico de concentração de defeitos igual a $1.3 \%$, vemos que a média da condutância decai de $85 \%$ quando a concentração de $C O$ vai de $0 \%$ para 50\%. Fazendo a mesma análise para o gráfico de concentração de defeitos igual a $0.5 \%$, a queda é de $50 \%$. Isso indica que o efeito da molécula sobre o sistema é mais facilmente detectado quando a concentração de defeitos do nanotubo é de $1.3 \%$.

Quando a concentração de defeitos é alterada de $1.3 \%$ para $0.5 \%$, a variação percentual da média da condutância (comparando um ponto de uma das curvas com o seu respectivo na outra curva) sofre uma queda que vai de $67 \%$ (com $0 \%$ de CO) a $90 \%$ (com $100 \%$ de $C O)$. Isso é devido ao aumento do número de regiões de espalhamento quando passamos de $0.5 \%$ para $1.3 \%$. Deve-se observar que o aumento da concentração de defeitos causa a diminuição de $\sim 3$ vezes no valor absoluto da condutância; por exemplo, a condutância decai de $0.17 G_{0}$ para $0.06 G_{0}$ com $0 \%$ de $C O$, o que poderia dificultar a medida. Portanto, deve-se ter um comprimisso entre a concentração de defeitos e a variação percentual 
da condutância em função da concentração do gás, afim de obter valores ótimos desses parâmetros para uma possível aplicação desse sistema como sensor de gás.

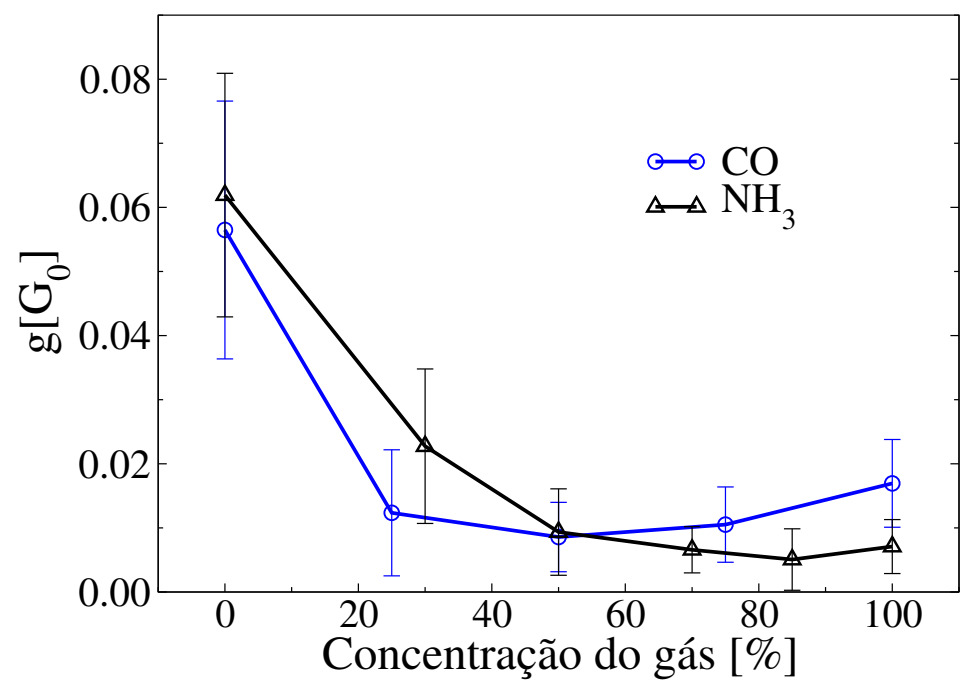

Figura 5.15: Média da condutância em função da concentração do gás para os sistemas $(N R+C O)$ e $\left(N R+N_{3}\right)$. A concentração de defeitos é igual a $1.3 \%$ e o comprimento do nanotubo de $220 \mathrm{~nm}$.

A Fig. 5.15 compara os cálculos com desordem dos sistemas $(N R+C O)$ e $\left(N R+N H_{3}\right)$ para concentração de defeitos igual a 1.3\%. O efeito do aumento da concentração do gás nos dois casos é semelhante e também a ordem de grandeza da condutância é a mesma. As curvas mostram diferenças, mas estas diferenças estão dentro das barras de erro associadas a cada ponto em que se calculou a média. Isso indica que, mesmo considerando os fatores de desordem nos cálculos das propriedades de transporte do sistema $N R+$ molécula, não parece ser possível distinguir entre $\mathrm{CO}$ e $\mathrm{NH}_{3}$ se o sistema fosse inserido em um ambiente contendo uma mistura desses gases. Embora o sistema não apresente 100\% de seletividade, os resultados indicam que ele poderia ser utilizado para detectar principalmente esses dois gases.

\subsection{Resumo dos resultados das propriedades de trans- porte}

Através dos resultados de propriedades de transporte apresentados na seção 5.1, verifica-se que há mudanças significativas na transmitância do sistema $N R$ devido à pre- 
sença de moléculas. Verifica-se que essas mudanças são mais expressivas para os sistemas que apresentaram maior transferência de cargas entre os átomos da molécula e os átomos da região do defeito. Isso pode ser visto quando comparamos as moléculas dissociadas com as adsorvidas, conforme apresentado na Tab. 4.5.

Os resultados apresentados na seção 5.2, indicam que através da análise do perfil da curva de transmitância e da variação percentual de corrente apresentadas para cada molécula (Fig. 5.13 e Tab. 5.1), não parece ser possível identificar a espécie química que está ligada ao nanotubo, comprometendo a seletividade do dispositivo. De fato, o que parece acontecer é que essas moléculas tendem a perturbar a região de defeito de maneira semelhante saturando os nitrogênios. Por exemplo, as curvas de DOS indicam que a presença das moléculas na região do defeito removem os estados dos átomos de nitrogênio para energias abaixo de $-1 e V$ em relação ao nível de Fermi. Ainda, o aumento(diminuição) nas DOS e PDOS nos intervalos de energia de $\pm 0.5 \mathrm{eV}$ indicam uma diminuição(aumento) na transmitância para o mesmo intervalo.

O efeito da desordem sobre o sistema $N R+N H_{3}$ [36] se mostrou importante, uma vez que foi possível explicar o comportamento experimental da condutividade [33]. Entretanto, na seção 5.3, vimos que o efeito da desordem sobre o sistema $N R+C O$ é o mesmo observado para o caso da amônia, de forma que mesmo levando em conta esse efeito nas simulações, não seria possível distinguir qual das duas moléculas estaria presente em uma possível aplicação como sensor de gás. Os resultados indicam que para a concentração de defeitos igual a 1.3\%, a variação percentual da média da condutância é maior, devido à presença de $C O$, quando comparada com a concentração de defeitos igual a $0.5 \%$. Isso indica que a presença da molécula seria mais facilmente detectada para uma concentração de defeitos de $1.3 \%$ do que de $0.5 \%$. 


\section{Capítulo 6}

\section{Conclusões e Perspectivas}

O presente trabalho teve por objetivo principal investigar teoricamente a possibilidade de aplicação, como sensor de gás, o sistema mostrado na Fig. 1.5. Esse sistema consiste de um nanotubo de carbono $(5,5)$ com uma divacância e 4 átomos de nitrogênio substitucionais. Ao longo do desenvolvimento do trabalho, as seguintes questões foram abordadas:

- Questão (1): através do estudo do processo de dissociação, quais dentre as moléculas mostradas na Fig. 1.6 se ligam na região de defeito do nanotubo e como se dá esse processo?

- Questão (2): através do estudo das propriedades de transporte, é possível saber se uma molécula se dissociou/ligou na região de defeito do nanotubo, de maneira que pudesse ser detectada?

- Questão (3): o sistema poderia ser utilizado como sensor de gás? Quais gases poderiam ser detectados se o sistema fosse inserido em uma atmosfera contendo uma mistura de gases?

- Questão (4): qual a importância de se considerar os fatores de desordem para simulações do sistema $N R$ como sensor de gás?

Para responder à questão (1):

Através de cálculos de estrutura eletrônica $(D F T)$, foram feitos estudos do processo de dissociação das moléculas mostradas na Fig. 1.6 para identificar quais dessas moléculas poderiam se ligar na região do defeito e, consequentemente, serem detectadas pelo sistema. 
Por ser computacionalmente caro, não foram calculadas as superfícies de potencial para cada sistema. Desta maneira, o procedimento adotado foi construir diferentes geometrias para cada sistema $N R+$ molécula e permitir a relaxação até atingir a estrutura de mais baixa energia. Seguindo esse procedimento, os resultados apresentados no capítulo 4, podem ser resumidos como segue:

(i) as moléculas $\mathrm{H}_{2} \mathrm{~S}, \mathrm{O}_{2}, \mathrm{H}_{2} \mathrm{O}$ e $\mathrm{H}_{2}$ constituem o grupo 1 de moléculas que poderiam se dissociar (já que a energia total do sistema após a dissociação é negativa, conforme Tab. 4.2) mas que o processo poderia apresentar barreira de potencial. Também para as moléculas $\mathrm{H}_{2} \mathrm{~S}$ e $\mathrm{H}_{2} \mathrm{O}$, algumas estruturas relaxadas apresentaram energias de ligação de $\sim-0.18 \mathrm{eV}$ e $\sim-0.3 \mathrm{eV}$, respectivamente. Esses energias estão associadas à formação de ligações de hidrogênio entre os átomos de hidrogênio das moléculas com os átomos de nitrogênio da região do defeito. Entretanto, a perturbação causada pelas moléculas fisisorvidas sobre a região de defeito do nanotubo é pequena quando comparada com os casos dissociados. Isso pode ser associado à transferência de cargas entre os átomos da molécula e os átomos do nanotubo, que é $\sim 10$ maior para o sistemas dissociados.

Para as moléculas $\mathrm{O}_{2}$ e $\mathrm{H}_{2}$ foram feitas outras relaxações em que variamos a altura da molécula em relação ao plano do defeito. Entretanto, quando colocamos a molécula em alturas arbitrárias, ela se afastou do nanotubo. Desta forma, os resultados indicam que deve haver uma barreira de potencial para que estas moléculas cheguem ao plano do defeito e se dissociem.

(ii) as moléculas $\mathrm{CO}_{2}$ e $\mathrm{N}_{2}$ constituem o grupo 2 que não se dissociam ou se ligam na região de defeito, uma vez que a energia de ligação para esses sistemas é positiva, por exemplo, igual a $2.95 \mathrm{eV}$ para a molécula de nitrogênio. Em particular, para a molécula de nitrogênio, este resultado é importante, uma vez que ela é uma dos principais constituintes do ar atmosférico. Em uma possível aplicação desse sistema como sensor de gás, o $N_{2}$, portanto, não seria detectado.

(iii) o grupo 3 é composto pelas moléculas $\mathrm{NH}_{3}$ e $\mathrm{CO}$ que se dissociam/ligam na região do defeito sem barreira. A primeira apresentou duas configurações de dissociação com energias de ligação iguais a $-0.59 \mathrm{eV}$ e $-0.26 \mathrm{eV}$. A segunda se ligou da mesma maneira em todos os casos testados, e a energia de ligação é de $-0.41 e V$. No caso da amônia, a 
estrutura de mais baixa energia em nossos cálculos foi a mesma encontrada no trabalho de Reily et al. [36].

Para responder à questão (2):

Por meio da teoria quântica de transporte eletrônico $(N E G F)$, foram calculadas as propriedades de transporte para cada sistema $N R+$ molécula que apresentou estruturas ligadas/dissociadas. Os resultados apresentados no capítulo 5, indicam que a dissociação das moléculas na região do defeito provoca mudanças significativas (que seria refletido na sensitividade do dispositivo) nas propriedades de transporte do sistema $N R$. Essas mudanças estão intimamente relacionadas com a saturação dos orbitais "p" dos átomos de nitrogênio do defeito pelos átomos da molécula, conforme as Fig. 5.2 e Fig. 5.3.

Para responder à questão (3):

Conforme discutido na seção 5.2, as mudanças nas propriedades de transporte para as diferentes moléculas dissociadas na região do defeito do nanotubo são muito semelhantes. Esse fato é um ponto fraco do sistema para uma possível aplicação como sensor de gás, já que a seletividade é uma característica que se busca em um sensor. Os resultados do cálculo da variação percentual da corrente no regime linear $(\Delta I)$, indicam que a amônia (para a estrutura com $E_{b}=-0.26 \mathrm{eV}$ ) seria a mais facilmente selecionada entre as demais, uma vez que na presença dessa molécula $\Delta I=-18.5 \%$. No entanto, a estrutura da amônia que apresentou energia de ligação $E_{b}=-0.59 \mathrm{eV}$, que é a mais provável de ocorrer, apresentou $\Delta I=-10.8 \%$. Esse valor de $\Delta I$ é muito próximo daqueles encontrados para as outras moléculas, inclusive para o $C O$.

De fato, as moléculas $\mathrm{NH}_{3}$ e $\mathrm{CO}$ foram as únicas que se dissociam/ligam à região de defeito sem barreira de potencial, assim, elas poderiam ser as mais facilmente detectadas pelo sistema $N R$. Em princípio, devido às propriedades de transporte desses sistemas (Fig. 5.10 e Fig. 5.11) serem muito semelhantes, parece não ser possível distinguir qual dessas moléculas estaria presente em uma atmosfera contendo uma mistura de apenas esses dois gases.

Para responder à questão (4):

Diante disso, foram investigadas as propriedades de transporte do nanotubo com $\mathrm{NH}_{3}$ e $C O$ adsorvidas na região do defeito considerando os fatores de desordem, o que permite simular sistemas mais próximos dos que se observa experimentalmente. Os resultados dos cálculos das médias das condutâncias em função da concentração do gás mostram 
que, a maior variação percentual da média das condutâncias em função da concentração do gás, ocorre entre as concentrações de $0 \%$ e $50 \%$ de CO. Considerando o gráfico de concentração de defeitos igual a 1.3\%, vemos que a média da condutância decai de $85 \%$ quando a concentração de $C O$ vai de $0 \%$ para $50 \%$. Fazendo a mesma análise para o gráfico de concentração de defeitos igual a $0.5 \%$, a queda é de $50 \%$. Isso indica que o efeito da molécula sobre o sistema é mais facilmente detectado quando a concentração de defeitos do nanotubo é de 1.3\%. Entretanto, deve-se observar que ao aumentar a concentração de defeitos de $0.5 \%$ para $1.3 \%$, o valor absoluto da média da condutância muda de $0.17 G_{0}$ para $0.06 G_{0}$, o que poderia dificultar uma possível medida.

Os resultados dos cálculos da média da condutância em função da concentração do gás, para os casos com $\mathrm{NH}_{3}$ e o $\mathrm{CO}$, mostraram o mesmo comportamento. Além disso, a ordem de grandeza da média da condutância nos dois casos é a mesma e os valores dessas médias também estão dentro das barras de erro associadas a cada ponto de média da condutância. Isso permite-nos concluir que não seria possível, mesmo considerando os efeitos de desordem, distinguir essas duas espécies, caso o sistema fosse inserido em um ambiente contendo uma mistura delas.

A grande maioria dos trabalhos teóricos encontrados na literatura sobre sensores de gás baseados em nanotubos de carbono não consideram os efeitos de desordem. Diante disso, o presente trabalho dá uma contribuição importante quanto à aplicação da metodologia apresentada na seção 3.2.3, a qual permitiu o estudo das propriedades de transporte de nanotubos de $\sim 220 \mathrm{~nm}$ contendo milhares de átomos, com defeitos aleatoriamente distribuídos e com diferentes concentrações de gases e defeitos.

Como perspectivas futuras seria interessante o estudo dos seguintes tópicos:

(i) calcular as superfícies de energia potencial para todos os sistemas $N R+$ molécula. Isso permitiria saber qual a probabilidade de ocorrer uma dissociação desses gases na região do defeito. Além disso, permitiria inferir quais as condições ótimas de aplicação desses sistemas em relação ao tipo de gás e à temperatura de operação.

(ii) refazer os cálculos das propriedades de transporte para as moléculas, principalmente do grupo 3, com voltagem externa aplicada, afim de verificar se, nessas condições, poderia se fazer a distinção entre o $\mathrm{NH}_{3}$ e o $C O$. Se sim, o sistema $N R$ poderia ser 100\% seletivo, dependendo das condições de operação. 
(iii) fazer os cálculos das propriedades de transporte para os sistemas desordenados considerando os dois gases. Em um primeiro momento, poderia fixar a concentração de defeitos ocupados pelos gases e variar a proporção entre esses gases (por exemplo, $30 \%$ de $\mathrm{CO}$ e $70 \%$ de $\mathrm{NH}_{3}$ ). Em seguida, poderia variar também a concentração dos defeitos ocupados pelos gases para uma determinada proporção dessas moléculas. Se através da aplicação de voltagem externa for possível distinguir essas espécies, possivelmente, poderia se ter um indicativo da concentração desses gases (mesmo em uma mistura), atráves das curvas das médias das condutâncias, obtidas com os cálculos das propriedades de transporte dos sistemas desordenados. 


\section{Apêndice A}

\section{Teoria do funcional da densidade -}

\section{DFT}

Em mecânica quântica aprendemos que toda informação que podemos ter de um determinado sistema está na função de onda total, $\tilde{\Psi}(\mathbf{R}, \mathbf{r})$, deste sistema, em que $\mathbf{R}$ e $\mathbf{r}$ são os conjuntos das coordenadas dos núcleos e dos elétrons, respectivamente. Nosso interesse é entender como a teoria do funcional da densidade se aplica no estudo da estrutura eletrônica de átomos, moléculas e sólidos.

Para iniciarmos vamos supor que o problema eletrônico possa ser separado da parte nuclear, de tal forma que a função de onda global seja escrita como $\tilde{\Psi}=\Phi(\mathbf{R}) \Psi(\mathbf{r} ; \mathbf{R})$, ou seja, a função de onda eletrônica $\Psi(\mathbf{r} ; \mathbf{R})$ depende explicitamente das coordenadas eletrônicas $\mathbf{r}$ e apenas parametricamente das coordenadas nucleares $\mathbf{R}^{1}$. Assim, sem levarmos em conta efeitos relativísticos, a função de onda de um único elétron sujeito a um potencial externo $v(\mathbf{r})$ é determinada pela equação de Schrödinger $^{2}$,

$$
\left[-\frac{\hbar^{2} \nabla^{2}}{2 m}+v(\mathbf{r})\right] \Psi(\mathbf{r})=\epsilon \Psi(\mathbf{r})
$$

Quando o sistema contém muitos elétrons, torna-se um problema de muitos corpos e a equação de Schrödinger a ser resolvida é

$$
\left[-\frac{\hbar^{2} \nabla^{2}}{2 m}+v\left(\mathbf{r}_{i}\right)+\sum_{i<j} U\left(\mathbf{r}_{i}, \mathbf{r}_{j}\right)\right] \Psi\left(\mathbf{r}_{1}, \mathbf{r}_{2} \ldots, \mathbf{r}_{N}\right)=E \Psi\left(\mathbf{r}_{1}, \mathbf{r}_{2} \ldots, \mathbf{r}_{N}\right)
$$

\footnotetext{
${ }^{1}$ Esta é a aproximação de Born-Oppenheimer [67]. Uma descrição mais detalhada desta aproximação pode ser vista na referência [68].

${ }^{2}$ Omitiremos a coordenada $\mathbf{R}$ para simplificar a notação.
} 
em que $N$ é o número de elétrons e $U\left(\mathbf{r}_{i}, \mathbf{r}_{j}\right)$ é a interação elétron-elétron. Como estamos interessados em sistemas de átomos, moléculas e sólidos que estão sujeitos à interação coulombiana, o operador energia potencial que descreve essa interação é o mesmo para todos os sistemas e é dado por

$$
\hat{U}=\sum_{i<j} U\left(\mathbf{r}_{i}, \mathbf{r}_{j}\right)=\frac{1}{4 \pi \epsilon_{0}} \sum_{i<j} \frac{e^{2}}{\left|\mathbf{r}_{i}-\mathbf{r}_{j}\right|}
$$

Da mesma forma, o operador energia cinética é o mesmo para sistemas não relativísticos e é escrito como

$$
\hat{T}=\sum_{i}-\frac{\hbar^{2} \nabla_{i}^{2}}{2 m}
$$

Na ausência de potenciais externos, o potencial $v(\mathbf{r})$ é dado pela interação elétron-núcleo de acordo com

$$
\hat{V}_{e x t}=\sum_{i} v\left(\mathbf{r}_{i}\right)=\sum_{i k} \frac{Z_{k} e^{2}}{\left|\mathbf{r}_{i}-\mathbf{R}_{k}\right|},
$$

em que $Z_{k}$ é o número atômico do átomo $k$ e $\mathbf{R}_{k}$ é a sua coordenada.

O esquema de solução dos sistemas eletrônicos antes da DFT se baseia em especificar o sistema escolhendo o potencial externo $\hat{V}_{\text {ext }}$ e introduzindo-o na equação de Schröedinger, então resolve-se essa equação e determina-se a função de onda $\Psi$. As propriedades do sistema são determinadas calculando-se os valores esperados dos respectivos operadores usando a função de onda encontrada $(\langle\Psi|\ldots| \Psi\rangle)$. Um desses observáveis é a densidade eletrônica

$$
\rho(\mathbf{r})=N \int d^{3} r_{2} \int d^{3} r_{3} \ldots \int d^{3} r_{N} \Psi^{*}\left(\mathbf{r}, \mathbf{r}_{2} \ldots, \mathbf{r}_{N}\right) \Psi\left(\mathbf{r}, \mathbf{r}_{2} \ldots, \mathbf{r}_{N}\right)
$$

\section{A.1 Teoremas de Hohenberg-Kohn}

Em 1964 Hohenberg e Kohn [69] com a enunciação de dois teoremas, consolidaram a teoria que considera a densidade eletrônica como uma variável fundamental ao invés de tratá-la como um observável. Assim, o esquema de solução dos sistemas eletrônicos pode ser resumido por

$$
\rho(\mathbf{r}) \Longrightarrow \Psi\left(\mathbf{r}, \mathbf{r}_{2} \ldots, \mathbf{r}_{N}\right) \Longrightarrow v(\mathbf{r})
$$


Embora existam outras formas de apresentação dos teoremas de Hohenberg-Kohn ${ }^{3}$, seguiremos a mesma maneira usada pelos autores em 1964 .

O primeiro teorema diz respeito à unicidade entre a densidade do estado fundamental $\rho_{0}(\mathbf{r})$ de um sistema de elétrons e o potencial externo $v(\mathbf{r})$ e é enunciado por:

Teorema I: A densidade $\rho_{0}(\mathbf{r})$ do estado fundamental de um sistema ligado de elétrons interagentes em um dado potencial externo $V_{\text {ext }}(\mathbf{r})$ determina unicamente $^{4}$ este potencial.

Em outras palavras, para cada potencial existe apenas uma densidade eletrônica para o estado fundamental e vice-versa. Além disso, se o estado fundamental for degenerado, o Teorema I refere-se à densidade de qualquer um desses estados. Como consequência imediata do Teorema I, tem-se:

Corolário: Se o potencial externo $V_{e x t}(\mathbf{r})$ é determinado, então também será o hamiltoniano e, consequentemente, as funções de onda de cada elétron para todos os estados (fundamental e excitados). Assim, uma vez conhecida a densidade $\rho_{0}(\mathbf{r})$ do estado fundamental, todas as demais propriedades do sistema estarão completamente determinadas.

Prova Do Teorema I: A demonstração desse primeiro teorema é feita por absurdo. Seja um estado fundamental de um sistema, caracterizado por um Hamiltoniano $\hat{H}$, contendo um potencial externo $v(\mathbf{r})$. A equação de Schroedinger é dada por:

$$
\left(\hat{T}+\hat{U}+\hat{V}_{e x t}\right) \Psi=E \Psi
$$

em que $\hat{T}, \hat{U}$ e $\hat{V}_{\text {ext }}$ são os operadores energia cinética, energia potencial de interação elétron-elétron e energia potencial externa, respectivamente. Vamos supor a existência de um outro potencial $V^{\prime 5}$ que dá origem a um Hamiltoniano $\hat{H}^{\prime}$ cuja solução é $\Psi^{\prime}\left(\hat{H}^{\prime} \Psi^{\prime}=\right.$ $\left.E^{\prime} \Psi^{\prime}\right)$, e que fornece a mesma densidade eletrônica $\rho(\mathbf{r})$. Pelo teorema variacional, temos as seguintes desigualdades:

$$
E=\langle\Psi|\hat{T}+\hat{V}+\hat{U}| \Psi\rangle\left\langle\left\langle\Psi^{\prime}|\hat{T}+\hat{V}+\hat{U}| \Psi^{\prime}\right\rangle\right.
$$

\footnotetext{
${ }^{3}$ Outras maneiras de apresentação dos teoremas da DFT podem ser encontrados na referência [70].

${ }^{4} \mathrm{O}$ termo unicamente pode ser entendido como a menos de uma constante aditiva.

${ }^{5}$ Omitiremos o subescrito ext do operador $\hat{V}_{\text {ext }}$ para simplificar a notação nas demonstrações dos teoremas.
} 


$$
E^{\prime}=\left\langle\Psi^{\prime}\left|\hat{T}+\hat{V}^{\prime}+\hat{U}\right| \Psi^{\prime}\right\rangle\left\langle\left\langle\Psi\left|\hat{T}+\hat{V}^{\prime}+\hat{U}\right| \Psi\right\rangle\right.
$$

Portanto,

$$
\begin{aligned}
& E<\left\langle\Psi^{\prime}|\hat{H}| \Psi^{\prime}\right\rangle=\left\langle\Psi^{\prime}\left|\hat{H}^{\prime}\right| \Psi^{\prime}\right\rangle+\left\langle\Psi^{\prime}\left|\hat{V}-\hat{V}^{\prime}\right| \Psi^{\prime}\right\rangle \\
& E<E^{\prime}+\int \rho(\mathbf{r})\left[v^{\prime}(\mathbf{r})-v(\mathbf{r})\right] d^{3} r .
\end{aligned}
$$

Desta forma, obtemos duas equações

$$
\begin{aligned}
& E<E^{\prime}+\int \rho(\mathbf{r})\left[v(\mathbf{r})-v^{\prime}(\mathbf{r})\right] d^{3} r \\
& E^{\prime}<E+\int \rho(\mathbf{r})\left[v^{\prime}(\mathbf{r})-v(\mathbf{r})\right] d^{3} r
\end{aligned}
$$

e chegamos na seguinte inconsistência

$$
E+E^{\prime}<E^{\prime}+E
$$

Então podemos verificar que existe apenas uma densidade eletrônica para o estado fundamental para o potencial escolhido. Em outras palavras, dado o arranjo atômico do sistema, a densidade eletrônica é conhecida e consequentemente o Hamiltoniano e as funções de onda.

O segundo teorema diz respeito a um funcional da energia com a densidade eletrônica para um determinado potencial externo e que pode ser enunciado como segue:

Teorema II: Existe um funcional ${ }^{6}$ para a energia em termos da densidade $E[\rho(\mathbf{r})]$, e a energia do estado fundamental corresponde ao mínimo global deste funcional para a densidade $\rho_{0}(\mathbf{r})$ do estado fundamental.

Corolário: $\mathrm{O}$ funcional $E\left[\rho_{0}\right]$ sozinho é suficiente para determinar exatamente a energia e a densidade do estado fundamental.

\footnotetext{
${ }^{6}$ Os operadores energia cinética $\hat{T}=\sum_{i}-\frac{\hbar^{2}}{2 m_{e}} \nabla_{i}^{2}$ e energia de interação elétron-elétron $\hat{U}=$ $\frac{1}{4 \pi \epsilon_{0}} \sum_{i<j} \frac{e^{2}}{\left|\mathbf{r}_{i}-\mathbf{r}_{j}\right|}$ são denominados universais, válidos para qualquer sistema eletrônico. Já o operador energia potencial $\hat{V}=\sum_{i} v\left(\mathbf{r}_{i}\right)$ é dito não-universal, pois depende do tipo de sistema em estudo. Como consequência, é por meio da energia potencial que se distingue o problema de um átomo frente ao de uma molécula, um sólido etc.
} 
Prova DO Teorema II:

O teorema estabelece que $E\left[\rho_{0}\right]$ é um funcional de $\rho(\mathbf{r})$ cujo valor mínimo é obtido através da densidade eletrônica do estado fundamental,

$$
E\left[\rho_{0}\right] \leq E[\rho]
$$

Como consequência, qualquer observável do sistema é um funcional único da densidade, portanto, a energia total para uma densidade $\rho(\mathbf{r})$ qualquer é dada por:

$$
\begin{aligned}
& E[\rho]=\langle\Psi[\rho]|\hat{T}+\hat{U}| \Psi[\rho]\rangle+\langle\Psi[\rho]|\hat{V}| \Psi[\rho]\rangle \\
& E[\rho]=F[\rho]+\langle\Psi[\rho]|\hat{V}| \Psi[\rho]\rangle,
\end{aligned}
$$

e para o estado fundamental tem-se,

$$
E\left[\rho_{0}\right]=F\left[\rho_{0}\right]+\left\langle\Psi\left[\rho_{0}\right]|\hat{V}| \Psi\left[\rho_{0}\right]\right.
$$

O funcional $F[\rho]$ é universal e é válido para qualquer sistema de $N$ elétrons. Para o estado fundamental, a energia pode ser expressa por:

$$
E\left[\rho_{0}\right]=F\left[\rho_{0}\right]+\left\langle\Psi_{0}|\hat{V}| \Psi_{0}\right\rangle
$$

Pelo teorema variacional, Eq. A.16, temos que

$$
\begin{array}{r}
E\left[\Psi_{0}\right]<E[\Psi] \\
\left\langle\Psi_{0}|\hat{T}+\hat{U}| \Psi_{0}\right\rangle+\left\langle\Psi_{0}|\hat{V}| \Psi_{0}\right\rangle\langle\langle\Psi|\hat{T}+\hat{U}| \Psi\rangle+\langle\Psi|\hat{V}| \Psi\rangle \\
F\left[\rho_{0}\right]+\left\langle\Psi_{0}|\hat{V}| \Psi_{0}\right\rangle<F[\rho]+\langle\Psi|\hat{V}| \Psi\rangle \\
E\left[\rho_{0}\right]<E[\rho]
\end{array}
$$

Os dois teoremas descritos acima mostram que é possível, a partir da densidade eletrônica, obter os observáveis do sistema. Entretanto, não diz nada a respeito de como isso pode ser feito.

\section{A.2 Aproximação de Thomas-Fermi}

A idéia de substituir a função de onda dos elétrons pela densidade eletrônica iniciou na década de 20 com os trabalhos de Thomas e Fermi [71, 72]. 
A aproximação de Thomas-Fermi supõe que o funcional da energia de interação entre os elétrons do sistema seja aproximadamente o termo de Hartree

$$
U[\rho(\mathbf{r})] \approx U_{H}[\rho(\mathbf{r})]=\frac{q^{2}}{2} \int d \mathbf{r} \int d \mathbf{r}^{\prime} \frac{\rho(\mathbf{r}) \rho\left(\mathbf{r}^{\prime}\right)}{\left|\mathbf{r}-\mathbf{r}^{\prime}\right|}
$$

ou seja, não leva em consideração a correlação e os efeitos de troca. Outra aproximação é considerar que o termo da energia cinética de um sistema de elétrons interagentes é o mesmo de um sistema de elétrons interagentes com densidade constante, $T[\rho(\mathbf{r})] \approx$ $T^{L D A}[\rho(\mathbf{r})]$. Isso corresponde a uma aproximação de densidade local conhecida como $L D A$. Nesse tipo de aproximação, supõe-se que o sistema não-homogênio real possa ser decomposto em células, cada uma com a densidade aproximadamente constante naquele volume.

Entretanto, mesmo com a aproximação local, a forma funcional para o termo $T[\rho(\mathbf{r})]$ não é conhecida. Para um sistema homogêneo não-interagente conhece-se uma forma funcional para o termo da energia cinética em função da densidade ${ }^{7}$ de forma que a aproximação final é considerar um sistema não interagente e também a aproximação local, que pode ser resumido por:

$$
T[\rho(\mathbf{r})] \approx T^{L D A}[\rho(\mathbf{r})] \approx T_{s}^{L D A}[\rho(\mathbf{r})]=T_{T F}
$$

A deficiência fundamental da aproximação de Thomas-Fermi faz com que seu uso seja limitado, sendo necessárias outras abordagens para um tratamento mais acurado de sistemas eletrônicos.

\section{A.3 Equações de Kohn-Sham}

Foram Kohn e Sham [73] que propuzeram uma maneira de efetuar esses cálculos, conhecido hoje como esquema de Kohn-Sham. A idéia central deste esquema é substituir o problema original complicado de muitos corpos interagentes por um problema equivalente de partículas independentes consideravelmente mais fácil de ser resolvido; isso é feito supondo que a densidade do estado fundamental do sistema original é a mesma de um

\footnotetext{
${ }^{7}$ Para um gás de elétrons homogênio, a densidade eletrônica está relacionada com a energia de Fermi por $\rho=\frac{1}{3 \pi^{2}}\left(\frac{2 m}{\hbar^{2}}\right)^{\frac{3}{2}} \epsilon_{F}^{3 / 2}$ e a energia cinética é $T=3 \rho \epsilon_{F} / 5$. Assim, a densidade de energia cinética é $t[\rho]=\frac{3}{5} \frac{\hbar^{2}}{2 m}\left(3 \pi^{2}\right)^{2 / 3} \rho^{2 / 3}$, de forma que a energia cinética na aproximação LDA de Thomas-Fermi é a integral em todo espaço-k da densidade de energia cinética: $T_{T F}=C_{k} \int \rho(\mathbf{r})^{5 / 2} d \mathbf{r}$.
} 
sistema auxiliar não interagente. Isso leva a equações de partículas independentes, em que a energia cinética é dividida em duas partes, uma que corresponde à energia cinética de elétrons não-interagentes $T_{s}[\rho]$ e a outra contendo a parte de correlação eletrônica $T_{c}[\rho]$. Em particular, o termo de energia cinética $T_{s}[\rho]$ continua sem forma funcional conhecida com relação à densidade eletrônica e se supormos a aproximação de densidade local, voltamos à mesma situação da Eq. A.26. Entretanto, em termos das funções de onda de uma partícula de um sistema não-interagente, o termo para o funcional energia cinética é escrito por:

$$
T_{s}[\rho]=-\frac{\hbar^{2}}{2 m} \sum_{i} \int d \mathbf{r} \psi_{i}^{*}(\mathbf{r}) \nabla^{2} \psi_{i}(\mathbf{r}),
$$

já que para sistemas não-interagentes, a energia cinética total é apenas a soma das energias cinéticas individuais. Como as funções de onda são funcionais da densidade (Teorema I), o termo de energia cinética é explicitamente um funcional dos orbitais e implicitamente um funcional da densidade eletrônica.

A energia potencial é dada pela soma de três termos: o primeiro é a energia de Hartree $U_{H}[\rho]$, o segundo é o termo de troca $U_{x}[\rho]$ e o último é o termo de correlação eletrônica $U_{c}[\rho]$. Desta forma a energia total é dada por

$$
\begin{array}{r}
E[\rho]=T_{s}[\rho]+U_{H}[\rho]+V[\rho]+E_{x c} \\
V[\rho]=\int \rho(\mathbf{r}) v(\mathbf{r}) d^{3} r
\end{array}
$$

As partes de correlação e troca $\left(T_{c}[\rho], U_{x}[\rho]\right.$ e $\left.U_{c}[\rho]\right)$ das quais não temos informação reunimos em um único termo chamado de energia de troca e correlação $E_{x c}$. Para encontrar a energia do sistema devemos miniminar o funcional $E[\rho]$ sujeito a um vínculo que é a conservação do número de elétrons (carga elétrica). Pelo método dos multiplicadores de Lagrange define-se uma nova função $F(\lambda, \mathbf{r})=f(\mathbf{r})-\lambda g(\mathbf{r})$, em que $\lambda$ será identificado como o potencial quimico. Devemos portanto calcular

$$
\delta\left(E[\rho]-\lambda\left[\int \rho(\mathbf{r}) d^{3} r-N\right]\right)=0
$$

assim,

$$
\begin{array}{r}
\frac{\delta T_{s}[\rho]}{\delta \rho}+\frac{\delta U_{H}[\rho]}{\delta \rho}+\frac{\delta V_{s}[\rho]}{\delta \rho}+\frac{\delta E_{x c}[\rho]}{\delta \rho}-\lambda=0 \\
\frac{\delta T_{s}[\rho]}{\delta \rho}+v_{H}[\rho](\mathbf{r})+v[\rho](\mathbf{r})+v_{x c}[\rho](\mathbf{r})-\lambda=0 .
\end{array}
$$


Considando então um sistema fictício de partículas não-interagentes sujeito a um potencial $v_{s}(\mathbf{r})$, a condição de minimização é escrita por

$$
\frac{\delta T_{s}[\rho]}{\delta \rho}+v_{s}[\rho](\mathbf{r})-\lambda=0
$$

já que na ausência de interações não existe troca e correlação bem como interação eletrostática. A densidade que fornece o mínimo dessa equação é $\rho_{s}(\mathbf{r})$. Comparando a Eq. A.32 com a Eq. A.31 vemos que para minimizar as duas equações com a mesma solução $\left(\rho_{s}(\mathbf{r}) \equiv \rho(\mathbf{r})\right)$, devemos ter

$$
v_{s}[\rho](\mathbf{r})=v_{H}[\rho](\mathbf{r})+v[\rho](\mathbf{r})+v_{x c}[\rho](\mathbf{r})
$$

Assim, pode-se calcular a densidade eletrônica de um sistema interagente (Eq. A.2) sujeito a um potencial $v(\mathbf{r})$, resolvendo as equações de um sistema não-interagente sujeito a um potencial $v_{s}(\mathbf{r})=v_{\text {eff }}(\mathbf{r})$. Em particular, a Equação de Schröedinger de uma partícula do sistema fictício é dada por

$$
\left[\frac{-\hbar^{2}}{2 m} \nabla^{2}+v_{e f f}(\mathbf{r})\right] \phi_{i}=\epsilon_{i} \phi_{i}
$$

em que as soluções são os orbitais de Kohn-Sham que reproduzem a densidade $\rho(\mathbf{r})$ do sistema original

$$
\rho(\mathbf{r})=\sum_{\text {ocup }} \phi_{i}^{*} \phi_{i}
$$

Em outras palavras, o esquema de Kohn-Sham supõe que existe um hamiltoniano que corresponde a um sistema de partículas não-interagentes sujeitas a um potencial efetivo que gera a mesma densidade do sistema interagente. Como o potencial $v_{\text {eff }}(\mathbf{r})$ depende da densidade eletrônica $\rho(\mathbf{r})$ e vice-versa, a solução deve ser obtida através de um cálculo autoconsistente. O processo começa com uma densidade de teste, então calcula-se o potencial $v_{s}(\mathbf{r})$, em seguida resolve-se a Eq. A.34 para determinar as funções $\phi_{i}$ e com a Eq. A.35 calcula-se uma nova densidade. O processo é repetido até que a densidade de entrada seja igual à de saída dentro de um critério de convergência.

O conjunto das Eq. A.33, Eq. A.34 e Eq. A.35 são conhecidas como equações de Kohn-Sham. Resolvendo essas equações, chega-se a uma expressão para a energia total do sistema no estado fundamental dada por:

$$
E_{0}=\sum_{i}^{N} \epsilon_{i}-\frac{q^{2}}{2} \int d^{3} r \int d^{3} r^{\prime} \frac{\rho_{0}(\mathbf{r}) \rho_{0}\left(\mathbf{r}^{\prime}\right)}{\left|\mathbf{r}-\mathbf{r}^{\prime}\right|}-\int d^{3} r v_{x c} \rho_{0}(\mathbf{r})+E_{x c}\left[\rho_{0}\right]
$$


O principal ganho do esquema de Kohn-Sham é que o termo da energia cinética é resolvido exatamente, mas introduz o problema de resolver $\mathrm{N}$ equações de uma partícula ao invés de minimizar o funcional da energia em relação à densidade.

\section{A.4 Aproximações $L D A$ e $G G A$}

A Eq. A.36 permite calcular a energia total de um sistema interagente. Entretanto, não é conhecida uma forma funcional para o termo de troca e correlação $\left(E_{x c}\right)$, desta maneira, são necessárias algumas aproximações para resolvê-lo. As aproximações mais utilizadas para resolver esse termo são:

- LDA(local density aproximation) [74]: Consiste em supor que o valor da energia de troca e correlação em um ponto de densidade $\rho(\mathbf{r})$ do sistema estudado é a mesma energia de troca e correlação de um gás de elétrons homogênio para o mesmo valor de densidade,

$$
E_{x c}^{L D A}[\rho]=\int \varepsilon_{x c}(\rho(\mathbf{r})) \rho(\mathbf{r}) d \mathbf{r} .
$$

Como consequência, o termo de troca e correlação da Eq. A.31 pode ser escrito por

$$
\begin{aligned}
v_{x c}[\rho] & =\frac{\delta E_{x c}[\rho]}{\delta \rho} \\
\frac{\delta E_{x c}[\rho]}{\delta \rho} & =\frac{d}{d \rho}\left(\varepsilon_{x c}(\rho) \rho(\mathbf{r})\right)
\end{aligned}
$$

em que $\varepsilon_{x c}$ é a energia de troca e correlação por elétron. O funcional de troca e correlação da Eq. A.37 é exato para um gás de elétrons homogênio, portanto é uma boa aproximação para sistemas nos quais a densidade eletrônica varia lentamente. Entretanto, essa aproximação ignora correções da energia de troca e correlação em um determinado ponto $r$ do espaço devido a possíveis inomogeneidades da densidade eletrônica em pontos vizinhos a $r .^{8}$

- GGA(Generalized gradient aproximation) [75]: Leva em conta a não homogeneidade da densidade eletrônica através do gradiente da densidade. Neste trabalho utilizamos o funcional de troca e correlação GGA-PBE. Para maiores detalhes ver [70].

\footnotetext{
${ }^{8}$ Vale observar que essa aproximação difere da aproximação de densidade local na abordagem de Thomas-Fermi, pois a primeira é para o termo de troca e correlação e a última para o termo de energia cinética.
} 


\section{Apêndice B}

\section{Otimização de parâmetros do SIESTA para o nanotubo $(5,5)$}

Antes de efetuarmos os cálculos de estrutura eletrônica dos sistemas pretendidos nesse trabalho, fizemos um estudo da influência de alguns parâmetros exigidos pelo programa SIESTA [37] sobre a energia total do nanotubo $(5,5)$ (Fig. B), afim de determinar quais os valores ótimos destes parâmetros para a realização das simulações. A partir de uma célula

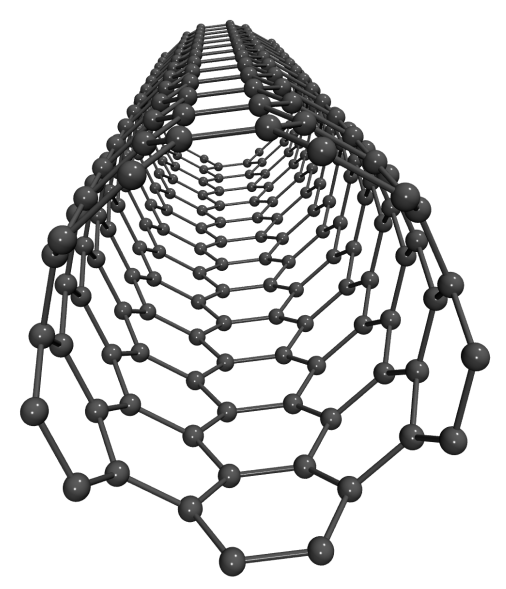

Figura B.1: Estrutura do nanotubo $(5,5)$ armchair.

unitária contendo 20 átomos de carbono e utilizando o valor experimental do parâmetro de rede ${ }^{1}$ do nanotubo $(5,5)$ que é de $2.46 \AA$ [76], foram feitas as otimizações dos parâmetros

\footnotetext{
${ }^{1}$ Deve-se observar que o parâmetro de rede é uma grandeza física característica de cada material que pode ser determinada experimentalmente. Não pertence à mesma categoria de parâmetros que o código SIESTA requer para as simulações.
} 
pontos- $k$ e MeshCutoff para esse sistema. O SIESTA usa condições periódicas de contorno

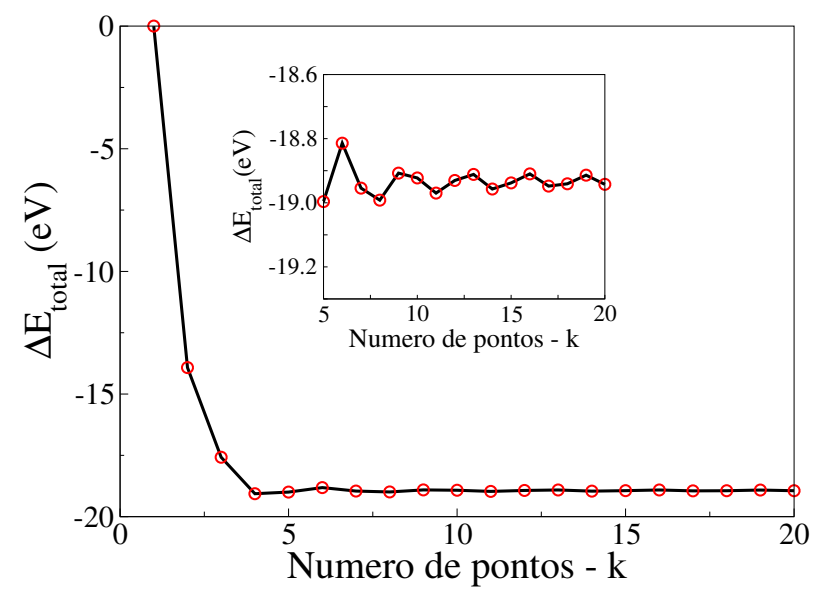

Figura B.2: Variação da energia total do sistema nanotubo $(5,5)$ em função do número de pontos-k. Na figura inserida, está mostrado especificamente como a energia varia na região de 5 a 20 pontos- $k$.

para resolução da equação de Schröedinger no espaço recíproco. Para a determinação da densidade eletrônica, essa equação deve ser resolvida para diferentes pontos- $k$ tomados na $1^{\underline{0}}$ zona de Brillouin. A Fig. B mostra a dependência da energia total do sistema à medida que o número de pontos- $k$ varia.

Outro parâmetro analisado foi o MeshCutoff que é equivalente à energia de corte que define o truncamento de uma expansão em termos de ondas planas. Quanto maior esse parâmetro mais refinado é o "grid" do esparço real para integração. A Fig. B mostra um gráfico da variação da energia total do sistema em função do MeshCutoff com 6 pontos- $k$ e o parâmetro de rede experimental. Pode-se perceber que a partir de 4 pontos-

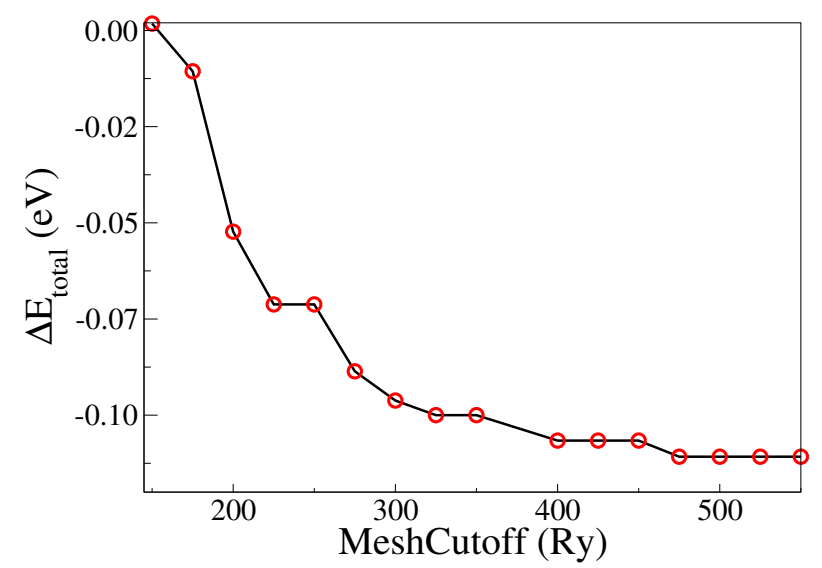

Figura B.3: Variação da energia total do sistema nanotubo $(5,5)$ em função do MeshCutoff. 
$k$ a energia já converge e que esse valor poderia ser usado com segurança. Ao mesmo tempo, o MeshCutoff parece convergir lentamente e que usar valores abaixo de 250Ry não seria adequado, já que a energia total varia muito nessa região. Entretanto, devemos levar em consideração o aumento do custo computacional à medida que usamos valores maiores desse parâmetro, conforme a Fig. B.4. Por exemplo, o custo computacional dobra comparando valores 350Ry e 600Ry, enquanto que a energia varia apenas $0.001 \%$.

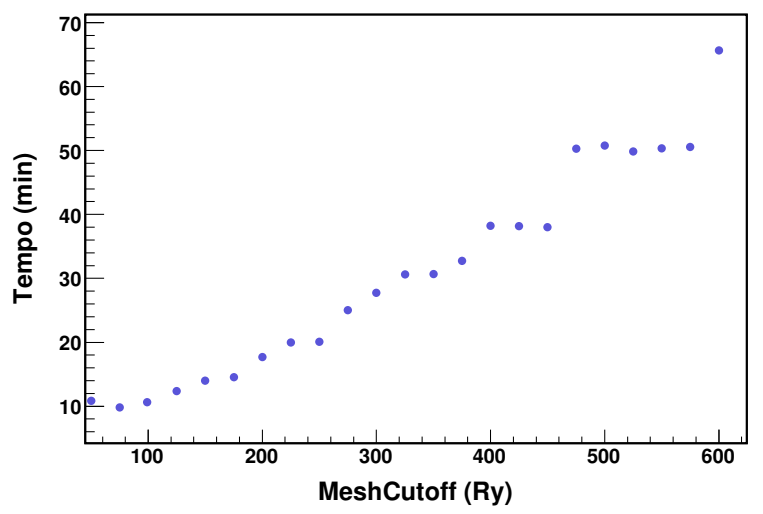

Figura B.4: Custo computacional em função do MeshCutoff.

Finalmente, usamos os parâmetros otimizados e otimizamos o parâmetro de rede do nanotubo de carbono $(5,5)$ em função da energia total do sistema. Foram feitos vários cálculos com diferentes parâmetros de rede (ao longo do tubo) ${ }^{2}$ dos quais pudemos construir um gráfico da variação da energia total do sistema em função deste, conforme mostrado na Fig. B.5. O valor do parâmetro de rede encontrado que minimiza a energia total do sistema foi de $2.49 \AA$, que difere de aproximadamente $2 \%$ do valor experimental

Além dos parâmetros descritos acima, o código SIESTA requer outros para a realização dos cálculos de estrutura eletrônica. Alguns dos mais relevantes e seus valores utilizados nesse trabalho estão listados abaixo:

- Conjunto base DZP

- Alcance da base definido com Energy-Shift de 0.03eV

- MeshCutoff de 350Ry

\footnotetext{
${ }^{2}$ As ligações de carbono dos átomos do nanotubo permaneceram livres para variar durante as simulações.
} 


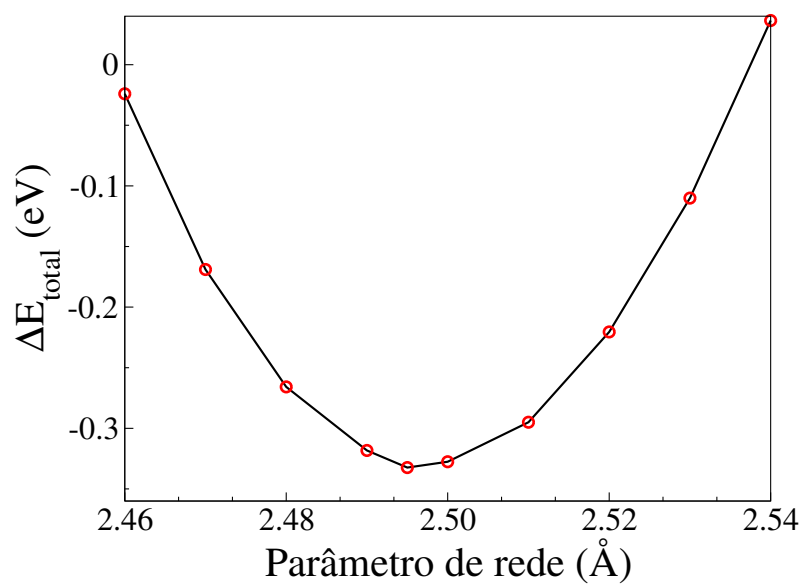

Figura B.5: Variação da energia total em função do parâmetro de rede.

- 6 pontos- $k$ na direção z : Deve-se enfatizar que o sistema estudado consiste de um nanotubo $(5,5)$ com defeito de nitrogênio.

- Tolerância para forças de $0.02 \mathrm{eV} / \AA$

- Funcional de troca-correlação GGA-PBE

- Todos os cálculos foram feitos levando em conta os graus de liberdade de spin 


\section{Referências Bibliográficas}

[1] J. Watson e K. Ihokura. MRS Bull. 24, 14 (1999).

[2] H. Nakagawa, S. Okazaki, S. Asakura, K. Fukuda, H. Akimoto, H. Takahashi e S. Shigemori. Sensors and Actuators B 65, 133 (2000).

[3] C. Wu-Hsun e L. Wen-Jin. J. Lab. Clin. Med. 133, 3182 (1999).

[4] M. D. Trotter, M. J. Sulway e E. Trotter. Clin. Chim. Acta 35, 137 (1971).

[5] S. V. Ryabtsev, A. V. Shaposhnick, A. N. Lukin e E. P. Domashevskaya. Sensors and Actuators B 59, 26 (1999).

[6] C. Di Natale, M. Antonella, M. Eugenio, R. Paolesse, G. D’Arcangelo, C. Roscioni, A. Finazzi-Agrò e A. D'Amico. Biosensors and Bioelectronics 18, 2003 (2003).

[7] A. Manolis. Clin. Chem. 29, 5 (1983).

[8] H. Kaji, M. Hisamura, N. Saito e M. Murao. Clin China Acta 85, 279 (1978).

[9] Duk-Dong Lee e Dae-Sik Lee. IEEE Sensors Journal 1, 214 (2001).

[10] T. Oyabu e M. Honda. Sensors and Actuators B 13, 462 (1993).

[11] V. V. Sysoev, J. Goschnick, T. Schneider, E. Strelcov e A. Kolmakov. Nano Lett. 7, 3182 (2007).

[12] N. Yamazoe. Sens. Actuators B 108, 374002 (2005).

[13] Y. T. Jang, S. I. Moon, J. H. Ahn, Y. H. Lee e B. K. Ju. Sens. Actuators B 99, 118 (2004).

[14] S. Iijima. Nature 354, 56 (1991). 
[15] P. Offermans, M. Crego-Calama e S. H. Brongersma. Nano Lett. 10, 2412 (2010).

[16] Y. Cui, Q. Wei, H. Park e M. C. Lieber. Science 293, 1289 (2001).

[17] F. Schedin, A. K. Geim, S. V. Morozov, E. Hill, P. Blake, M. I. Katsnelson e K. S. Novoselovi. Nature Materials 6, 652 (2007).

[18] J. Kong. Science 287, 622 (2000).

[19] P. G. Collins, K. Bradley, M. Ishigami e A. Zettlm. Science 287, 1801 (2000).

[20] W. Wongwiriyapan, S. Honda, H. Konishi, T. Mizuta, T. Ikuno, T. Itoa, T. Maekaya, H. Suzuki, K. Ishikawa, K. Oura e M. Katayama. Japanese Jounal of Applied Physics 44, $482(2005)$.

[21] T. Zhang, M. B. Nix, Bong-Young Yoo, M. A. Deshusses e N. V. Myung. Eletroanalysis 18, 1153 (2006).

[22] J. Suehiro, G. Zhou e M. Hara. J. Phys. D: Appl. Phys. 36, 109 (2003).

[23] J. Zhao, A. Buldum, J. Han e J. P. Lu. Nanotechnology 13, 195 (2002).

[24] S. Peng, K. Cho, P. Qi e H. Dai. Chem. Phys. Lett. 387, 271 (2004).

[25] J. Tersoff, S. Heinze, R. Martel, V. Derycke, J. Appenzeller e Ph. Avouris. Phys. Rev. Lett. 89, 106801 (2002).

[26] J. Andzelm, N. Govind e A. Maiti. Chem. Phys. Lett. 421 (2006).

[27] S. Peng e K. Cho. Nano Lett. 3, 513 (2003).

[28] W. Q. Han, J. Cumings, X. Huang, K. Bradley e A. Zettl. Chem. Phys. Lett. 346, 368 (2001).

[29] J. M. García-Lastra, D. J. Mowbray, K. S. Thygesen, A. Rubio e K. W. Jacobsen. Phys. Rev. B 81, 245429 (2010).

[30] D. J. Mowbray, C. Morgan e K. S. Thygesen. Phys. Rev. B 79, 195431 (2009).

[31] Z. Zanolli e J. C. Charlier. Phys. Rev. B 80, 155447 (2009). 
[32] M. Terrones, P. M. Ajayan, F. Banhart, X. Blase, D. L. Carroll, J. C. Charlier, R. Czerw, B. Foley, N. Grobert, R. Kamalakaran, P. Kohler-Redlich, M. Ruhle, T. Seeger e H. Terrones. Applied Phys. A 74, 355 (2002).

[33] F Villalpando-Paez, A. H. Romero, E Munoz-Sandoval, L. M. Martinez, H. Terrones e M. Terrones. Chem. Phys. Lett. 386, 137 (2004).

[34] A. R. Rocha, M. Rossi, A., Fazzio e A. J. R. da Silva. J. Phys. D 43, 374002 (2010).

[35] M. R. Carvalho. Estudo Teórico de Sensores Baseados em Nanotubos $C N_{x}$, utilizando cálculos ab initio. Dissertação de mestrado, Instituto de Física da USP, (2007).

[36] A. R. Rocha, M. Rossi, A. J. R. da Silva e A. Fazzio. Phys. Rev. Lett. 100, 176803 (2008).

[37] E. Artacho, D. Sánchez-Portal, P. Ordejón, A. García e J. M. Soler. Phys. Stat. Sol. (b) 215, 809 (1999).

[38] H. W. Kroto, J. R. Heath, S. C. OB́rien, R. F. Curl e R. E. Smalley. Nature 318, $162(1985)$.

[39] K. S. Novoselov, A. K. Gueim, S. V. Morozov, D. Jiang, Y. Zhang, S. V. Dubonos, I. V. Grigorieva e A. A. Firsov. Science 306, 666 (2004). Supporting Online Material: http://www. sciencemag. org/cgi/content/full/306/5696/666/DC1.

[40] A. K. Gueim e K. S. Novoselov. Nat. Mat. 6, 183 (2007).

[41] Jean-Christophe Charlier, X. Blase e S. Roche. Rev. Mod. Phys. 79, 677 (2007).

[42] S. Iijima e T. Ichihashi. Nature 363, 603 (1993). Errata em Nature 363, 737 (1993).

[43] N. Hamada, S. Sawada e S Oshiyama. Phys. Rev. Lett. 68, 1579 (1992).

[44] M. S. Dresselhaus e G. Dresselhaus. Carbon Nanotubes: Synthesis, Structure, Properties and Applications. Springer, New York, NY, USA, (2000).

[45] H. M. Rümmeli, C. Kramberger e et al. Nanotechnology 17, 5469 (2006).

[46] T. W. Ebbesen. Production and purification of carbon nanotubes. CRC Press, Boca Raton, Fla, USA, (1997). 
[47] J. Li, Y. Lu, Q. Ye e et al. Nano Lett. 3, 929 (2003).

[48] P. Ayala, R. Arenal, A. Loiseau e A. Rubio. Rev. Mod. Phys. 82, 929 (2010).

[49] F. Villalpando-Paez e et al. Chem. Phys. Lett. 424, 345 (2006).

[50] A. L. Elías, P. Ayala, A. Zamudio, M. Grobosch, J. M. Cruz-Silva, E. abd RomoHerrera, J. Campos-Delgado, H. Terrones, T. Pichler e M. Terrones. J Nanosci Nanotechnol. 10, 3954 (2010).

[51] J. D. Vianna, A. Fazzio e S. Canuto. Teoria Quântica de Moléculas e SólidosSimulação Computacional. Editora Livraria da Física, São Paulo, 1ª edição, (2004).

[52] R. Landauer. IBM J. Res. Develop. 1, 233 (1957).

[53] M. Büttiker. Phys. Rev. Lett. 57, 1761 (1986).

[54] M. Büttiker. IBM J. Res. Develop. 32, 317 (1988).

[55] R. Landauer. Phys. Scr. 110, 1402 (1992).

[56] Daniel S. Fisher e Patrick A. Lee. Phys. Rev. B 23, 6851 (1981).

[57] A. R. Rocha e S. Sanvito. Electronic transport at the nanoscale: Theoretical and computational aspects. LAP Lambert Academic Publishing, Dublin, $1^{\underline{0}}$ edição, (2010).

[58] F. D. Novais, A. J. R. da Silva e A. Fazzio. Brazilian Journal of Physics 36, 799 (2006).

[59] S. Datta. Electronic Transport in Mesoscopic Systems. Cambridge University Press, Cambridge, $1^{\mathrm{a}}$ edição, (1995).

[60] M. Di Ventra. Electrical transport in nanoscale systems. Cambridge University Press, Cambridge, $1^{\underline{0}}$ edição, (2008).

[61] J. E. Padilha, A. R. Rocha, A. Fazzio e A. J. R. da Silva. Phys. Rev. B 77, 153406 (2008).

[62] E Artaxo, D. Sánchez-Portal, A. García e J. Soler. Physica Status Solidi B 215, 809 (1999). 
[63] T. H. Tang, S. J. Deretey, E. Knak Jensen e I. G. Csizmadia. Eur. Phys. J. D 37, 217 (2006).

[64] Y. Wei, A. C. de Dios e A. E. McDermott. J. Am. Chem. Soc. 121, 10389 (1999).

[65] M. S. Martin, M. J. Yonezawa, J. Horner, C. W. Macosko e D. M. Ward. Chem. Mater. 16, 3045 (2004).

[66] P. M. F. J. Costa, D. Golberg, M. Mitome e Y. Bando. Appl. Phys. A 90, 225 (2008).

[67] M. Born e J. R. Oppenheimer. Ann. Physik 84, 457 (1927). Traduzido por S. M. Blinder com correções de B. Sutcliffe e W. Geppert, em 23 de janeiro de 2002.

[68] E. H. Júnior. Estudo Teórico da evolução dinâmica de nanofios de ouro puro e com impurezas. Tese de doutorado, Instituto de Física da USP, (2009).

[69] P. Hohenberg e W. Kohn. Phys. Rev. B 136, 864 (1964).

[70] K. Capelle. A Bird's Eye View of Density-Functional Theory, arXiv, 18 Nov (2006). http://arxiv.org/pdf/cond-mat/0211443v5.

[71] L. H. Thomas. Proc. Cambridge Philos. Soc. 23, 542 (1927).

[72] E. Fermi. Rend. Lincei 6, 602 (1927).

[73] W. Kohn e L. J. Sham. Phys. Rev. 140, A1133 (1965).

[74] D. M. Ceperley e B. J. Alder. Phys. Rev. Lett. 45, 566 (1980).

[75] J. P. Perdew, K. Burke e M. Ernzerhof. Phys. Rev. Lett. 77, 18 (1996).

[76] G. Dresselhaus R. Saito e M. S. Dresselhaus. Physical Properties of Carbon Nanotubes. Imperial College Press, Londres, $1^{\mathrm{a}}$ edição, (1998). 